\title{
MASTER
}

LBL --22233

DE87 001883

\section{HELICALLY LINKED MIRROR ARRANGEMENT *}

\author{
PRABHAT RANJAN
}

Ph. D. Thests

\author{
Laurence Berkeley Laboratory \\ University of California
}

Berke 1ey,CA 94720

August, 1986

\section{DISCLAIMER}

This report was prepared as an account of work sponsored by an agency of the United States Government. Neither the United States Government nor any agency thereof, nor any of their employees, makes any warranty, express or implied, or assumes any legal liability or responsibility for the accuracy, completeness, or usefulness of any information, apparatus, product, or process disclosed, or represents that its use would not infringe privately owned rights. Reference herein to any specific commercial product, process, or service by trade name, trademark, manufacturet, or otherwise does not necessarily constitute or imply its endorsement, recommendation, or favoring by the United States Government of any agency thereof. The views and opinions of authors expressed herein do not necessarily state or reflect those of the United States Government or any agency thereof.

* This work was supported by the Director, Office of Energy Research, Office of Fusion Energy, of the U.S. Department of Energy under Contract NO. OE-ACO3-765F00098. 
Helically Linked Mirror Arrangement

Prabhat Ranjan

Ph. D. Thesis

Lawrence Berkeley Laboratory

University of California

Berkeley, CA 94720

\section{Abstract}

Although Tokamak devices are nearing the breakeven point for fusion energy production, there are still many hurdles in the way of technical and economic development before we may declare them to be the fusion reactor designs of the future. Thus we need not give up hope for alternate schemes which may cross these hurdles easier than the tokamak. We describe in this paper a scheme of helical linking of mirror sections, which endeavors to combine the better features of torotdal and mirror devtces by eliminating the longttudinal loss of mirror machines, having moderately high average $B$ and steady state operation.

Our scheme is almed at a device, with closed magnetic surfaces having rotational transform for equfliortum, one or more axisymmetric straight sections for reduced radial loss, a simple geometrical axis for the links and an overall positive magnetic well depth for stabllity. We start by describing several other attempts at linking of mirror sections, made both in the past and the present. Then a description of our helfcally linked mirror scheme is given. This example has three identical straight sections connected by three sections having helical geometric axes. A theoretical analysis of the magnetic field and single-particle orbits in them leads to the 
conclusion that most of the passing particles would be confined in the device and they would have orbits independent of pitch angle under certain conditions. The magnetic rotational transform is estimated to be $310^{\circ}$ with circular magnetic surfaces. Then an estimate of maximum equilibrium $B$ is made which leads to a value of $5.5 \times$ on the basis of transverse displacement of the plasma column. This may mean a $B$ value of 15-20 $\mathrm{x}$ in the straight sections with lower magnetic field. A scheme of rotating nonctrcular magnetic surfaces to produce vanishing charge neutralization current is described, though not numerically tested.

Numerical results are then presented, which agree well with the theoretical results as far as passing particle orbits are concerned. The rotational transform value is shown to be very close to the estimated value. The particles trapped in the helical sections are found to get lost fast. A disussion of this on the overall confinement and density is given in appendix-B. Use of noncircular colls in the helical links to produce an average magnetic well is also studied. The magnetic field lines still form nested magnetic surfaces. However, the depth of the magnetic well is positive only near the magnetic axis for the set of colls used.

Some consideration on the stability of linked mirrors is presented. It is concluded that this scheme can be useful if a suttable means of stabllizing the system and reduction of charge neutralization current can be achieved. Use of modular coils is also suggested. 
Table of Contents

\begin{tabular}{|c|c|c|}
\hline & & Page \\
\hline Title Page & & 1 \\
\hline Abstract & & $i \mathfrak{i}$ \\
\hline Table of Contents & & iv \\
\hline Acknowl edgements & & vi \\
\hline Dedication & & viti \\
\hline List of Symbols & & $1 x$ \\
\hline List of Figures & & $x$ \\
\hline \multirow[t]{4}{*}{ Chapter I } & Introduction to Fuston & 1 \\
\hline & Magnetic Confinement & 3 \\
\hline & Magnetic Mirror & 5 \\
\hline & Toroidal Confinement & 7 \\
\hline \multirow[t]{7}{*}{ Chapter II } & Review of Toroidally Linked Mirror & \\
\hline & Conftgurations & 11 \\
\hline & Stellarator-Mirror Machine Target Plasma & \\
\hline & Reactor & 11 \\
\hline & Toroldally Linked Mirrors & 14 \\
\hline & Linked Baseball Cotls & 18 \\
\hline & Dracon Conftnement & 18 \\
\hline Chapter III & Description of Helically Linked Mirrors Scheme & 24 \\
\hline Chapter IV & Theoretical Analysis & 29 \\
\hline Cnapter V & Estimate of Maximum Equififbrium B & 37 \\
\hline Chapter VI & Charge Neutralization Current & 48 \\
\hline
\end{tabular}


Vacuum Magnetic fleld Properties

Single Particle Motion

Noncircular Colls

65

Chapter VIII

Stability of Linked Mirrors

73

Chapter IX

Summary and Concluston

Appendix A

Mercier Coordinate System

81

Appendix B

Oiscusston of Particles Trapped in the Helical

Sections

Append $1 \times \mathrm{C}$

Generation of Colls

References 


\section{Acknowledgements}

At the end of this dissertation. I come to the most pleasurable Job of acknowledging my sincere thanks to varlous persons, who have helped me through the five years of my. stay in the United States. First of all, I wish to thank my research advisor Prof. Wulf $B$. Kunkel for letting me work on a stimulating research topic and providing me guidance with the personal support and encouragement. I would also like to acknowledge here, the painstaking task he took of improving the language and the content of my thesis in the shortest possible. time. I would also like to thar.k Prof. Lichtenberg and Prof. Mozer, my Thes is Commitee members, who read through my thesis in very short time. I would also express my gratitude to a large number of faculty members in the Physics and other departments, who have helped me through my course work and teaching assignments. My thanks also go to Caroll Tung and Ken Miller. Physics department graduate assistants, for helping me through the maze of the University bureaucracy.

I would also like to thank Norm Abt and Alicia Ehrhardt(PPPL) for providing me with the source code of their computer programs. I would also like to thank Gary Guethlein, John Trow, Jeff Stutzin, Ludmilla Soroka, Joe Kwan, Rick Steele and all the members of the MFE group. who have helped me in more ways than I can mention. I would also like to acknowledge my gratitude to Martha Duenas (who also carried out the gigantic task of typing the thesis through the various versions Whout a strain on her face). Tina Aitkens and Bill Schraff for 
providing me with the secretarial and many other types of assistance through the three years of my stay at the Lawrence Berkeley Laboratory. And last, but not the least, I would like to thank my wife Sutapa, for her constant encouragement and support and for adjusting to my odd working hours during the last six months. She is also responstble, through her numerous reminders, for completion of my thesis in time. My thanks also go to my daughter, little Juhi. Through her tireless attempts at whatever she wants, she has been a constant source of entertainment and inspiration. It would not have been possible to reach this stage of writing a Ph. D. thesis in a forelgn country but. wt thout the constant support of my parents, my brothers and other family members. I dedicate this dissertation to my grandmother, who brought me up in my early childhood, and has been a living example of a hardworking person, even in her old age. 


\section{Dedicotion}

To my Grandmother 


\section{List of Important Symbols}

Symbol Description

First occurred

on page

II Component parallel to magnetic field 5

1 Component perpendicular to magnetic field 5

B Ratio of plasma pressure to fleld energy density 9

* Curvature 20

$x$ Torsion 20

c Eccentricity 20

Yv Velocity Ratio. 20

- Magnetic scalar potential 29

- Transverse coordinate axis in Mercier Coordinates 29

S Axtal length 29

w Angular coordinate axis 29

a Radius of colenoid 29

* Function of magnetic surface 30

1 Rotattonal transform 32

b Outer radius of plasma column 39

5 Displacement of magnetic surface 40

ra Radlal distance 59

i Unit vector along tangential direction 81

D Unit vector along principal normal 81

b Unit vector along binormal 


\section{List of Figures}

Fig. 1.1

Fig, 2.1

Fig. 2.2

Fig. 3.1

Fig. 3.2

Fig. 4.1

Fig. 5.1

Fig. 5.2

Fig. 7.1

Fig. 7.2

FIg. 7.3

Fig. 7.4

F1g. 7.5

Fig. 7.6

Fig. 7.7
Magnetic field strength in magnetic mirror. Stellarator mirror machine hybrid scheme magnetic fleld. [From Dawson et al. (1971)]

Schematic diagram of Stellarator-Mirror machine hybrid. [From Dawson et al. (1971)]

Coil arrangement of Helically Linked Mirror. Comparative magnetic field strength in THELMA. Expected magnetic suriaces in THELMA. Approximation to curvature for estimating equtitibrium $B$.

$B$ vs. maximum displacement of plasma column. Magnitude of $\bar{\delta}$ along the magnetic axts with no magnetic mirror.

Magnitude of $B$ along the magnetic axis with magnetic mirror.

Puncture plot at middle of the helical section with circular cotls.

Puncture plot at middle of the straight section with ctrcular cotl.

Rotational transform vs. radial distance with circular cotls.

rypical plot of gutding center radtal distance along geometrical axis.

Radial variation of guiding center orbit puncture plot. 
Fig. 7.8

Fig. 7.9

Fig. 7.10

Fig. 7.11

Fig. 7.12

Fig. 7.13

Fig. 7.14

Fig. A.1

Fig. B.1

Fig. C.1

Fig. C.2

Fig. C.3

Fig. $C .4(a)-(f)$
Variation of guiding center orbit puncture plot with sign of charge.

Variation of guiding center orbit with pitch angle. Puncture plot at the middle of the ielical section with noncircular collis.

Puncture plot at the middle of the straight section with noncircular colls.

Rotational transform vs. radial distance for noncircular coils.

Stability $B$ from shear vs. radial distance for noncircular coils.

Magentic well vs. radial distance for noncircular cotls.

Mercier coordinate system related figures

plot of radial distance along axis for particle

trapped in the helical section.

Parameters associated with a helix

looking from the top at the geometrical placement of sides.

Looking at the straight sections along the axis of generating cylinder.

Shapes of vartous noncircular colls with the Monticello bean parameters. 
Chapter I

Introduction to Fusion

From the late 1920's, when it was suggested that energy of the sun is released by thermonuclear reactions (also referred to as 'fustion' reactions), the great desirability of making this type of reaction possible in the laboratory has been recognised [Teller (1981)]. One of the main attractions of controlled fusion is the fact that deuterium, a main ingredient of proposed fusion reactors, occurs naturally with an abundance of one part in six thousand of hydrogen. Thus considering the amount of water in the oceans, it has been estimated that we have sufficient fuel to last for twenty thousand million years! [Bishop (1958)]. The cost of extracting deuterium from water is a tiny fraction of the cost of conventional fuels. A second feature of the controlled fusion reactors is the inherent safety of the reactors. Since only very small amounts of fuel would be within the reactor at any instant of $t$ tme, there would be no possibility of an explosion or a "runaway" reaction. A third consideration is that, in contrast to the case of fission, a fusion reactor would present a relatively small problem of disposal of radioactive by-products. The products of fusion, if the nuclear burning were carrled to its proper completion, would consist of nonradioactive nuclet only. (However the controlled fusion reactor itself and the surrounding structure would become radioactive due to intense flux of neutrons). An additional point of potentially great significance is the possibility of direct generation of electric power, which could eliminate the costly and inefficient stage of a thermal cycle. 
However, unlike fission, the first seif-sustained fusion reactions on the earth occurred in an uncontrolled explosion in the form of the 'Hydrogen Bomb'. The attempt to perform this reaction in a controlled form, which could be used to generate useful energy, has proved much mare complex than thought initially. At this point, we would like to point out some of the striking similarities and differences between thermonuclear explosions and thermonuc lear reactors.

The point of similarity is obvious. In both cases we deal with thermonuclear fusion, f.e., reactions where exceedingly high temperatures, of the order of $10^{\circ} \mathrm{K}$, permit light nuclei to approach each other closely enough so that energy may be liberated by synthesizing heavier nuclear spectes from the smallest nuclei, usually hydrogen isotopes. In the sun itself the primary reaction is between two protons, a reaction so slow that with extreme difficulty it has been observed in a laboratory. But at this point we have some practical alternatives avallable in the form of reactions involving deuterium and tritium, as follows:

$$
\begin{aligned}
& D+D+T+p+4.0 \mathrm{MeV}, \\
& D+D \rightarrow \mathrm{He}^{2}+n+3.3 \mathrm{MeV}, \\
& D+T \rightarrow \mathrm{He}^{\circ}+n+17.6 \mathrm{MeV} .
\end{aligned}
$$

These play the essential role in both the explosion and the controlled release of fuston energy.

On the other hand, the difference between explosive and controlled release of fustion energy is, from a practical point of view, enormous. In the former there is no need to confine the reaction; in the latter, this problem is all important. Incidentally, this difference is present 
In the case of fission reactions also. However, in that case the reaction rate depends not only on the density of the fuel but also on the slowing down rate of the neutrons released. The latter makes this matter simpler to deal with by using various types of moderators. In the case of fusion, the situation is quite different. The essential condition for the reaction is a high temperature and at the millions of degrees simply containing the fustonable material in a closed volume, separate from the wall presents a formidable problem. In the case of sun this is done by simple gravitational force, which becomes strong enough to hold the materlal together.

So far there have been two opposite approaches by which attempts are being made to turn controlled fusion into practical reality. One approach is to use the fuel at great dilution and thereby slow down the reaction so that it can be reasonably guided. In this approach we use densities of approximately $10^{24}-10^{35}$ of hydrogen isotopes per $\mathrm{cm}^{3}$. The other approach is to bring fuel to extremely high densities, more than a thousand times that of liquid hydrogen. Explosions similari to those occurring in the hydrogen bomb can be produced, though the individual explosions have less than one-millionth the output of a typical hydrogen bomb. Repeating this process can lead to nuclear energy production analogous to that of an internal combustion engine. Historically magnetic confinement was the first approach to be tried in the early 1950 s.

Magnetic Confinement

In the magnetic confinement approach an appropriate magnetic field is used so that the plasma is confined in a closed yolume and does not go 
and strike the walls with high energy. However interactions between plasmas in the magnetic field is subject to a variety of instabilities. By now considerable progress has been made towards understanding and limiting the effects of instabilities. At this time many plasma physicists believe that most of the plasma instabllity growth can be controlled so that their effect is not catastrophic on confinement. Thus it appears that a self-supporting controlled fusion process will be produced in the near future.

A steady reaction in a low-density plasma requires that the energy gain and energy loss should balance within the plasma. The two main balancing effects are the following: on the one hand, energy production in the thermonuclear reactions together with deposition of this energy in the plasma- which means that the energetic, positively charged-particle reaction products share their energy with the particles in the plasma, Including the electrons; on the other hand there is energy loss due to loss of plasma and the energy loss due to radiation and to other energy transport. The loss of plasma can be minimized, but the loss due to radiation becomes a high portion of the plasma energy production at high temperatures. Several suggestions have been made on how we may be able to use the energy going into radiation in some useful way. A quantitative estimate which is used to express this balance is the so-called 'Lawson iriterion'. According to this the energy input equals the energy output from the fusion reaction, if the product of plasma density $(n)$ and confinement time $(\tau)$ is equal to the Lawson Number ( $10^{24}$ per $\mathrm{cm}^{3} \mathrm{Sec}$ ) for a fuel of deuterium and tritium. However this must be at the required temperature for the fusion reactions to take 
place. At the present state of experiments we have come very close to this number, but not yet achieved it. However the goal of fuston research is to exceed this 11 mit by a sufficient amount, so that a possibly self-sustaining fuston reaction can be maintained along with net economic outplit of power.

On the basis of the properties of field lines the approach to magnetic confinement can be broadly classified under two classes. One of them is the so called 'Torotidal Devices' or 'Closed Configurations', in which the field lines remain mostly confined to a closed volume in the form of a torus. The other is the so called 'Mirror Devices' or 'Open Configurations', in which the ffeld lines leave the system, and may extend to infinity, and the major component of the magnetic fleld is a long a linear dimension.

Magnetic Mirror: In the magnetic mirror concept, the plasma is confined in a straight tube by means of an externally imposed axial magnetic field, with magnetic mirrors to inhibit the loss of particles out of the ends of the tube. The magnetic field is arranged such that the field is relatively weak in the central region and strong at the two ends. The strong field at the ends constitute "magnetic mirrors" which tend to repel charged particles of the plasma and (under certain conditions) reflect them completely back towards the central region. Specifically, if $\varepsilon_{1}$ is the energy of a particle in a plane perpendicular to the magnetic field and $E_{\|}$its energy parallel thereto, then the particle will be reflected by a magnetic mirror if

$$
E_{\|} / E_{\perp} \leqslant\left[B_{m} / B_{0}-1\right] \text {. }
$$


where $B_{m}$ and $B_{0}$ are the field strengths at the mirror (See Fig. 1.1) and in the central region respectively. Expressed another way, it may be seen that a particle will be able to escape through the mirrors if its velocity-vector lies in a cone (the so-called "Loss Cone") whtch has a half-angle $\theta$ given by

$$
\theta=\sin ^{-1} r\left(B_{0} / B_{m}\right) \text {. }
$$

One of the ways, which has been suggested to impede the rapid plasma loss along the field lines, is the use of electrostatic potential hills at the ends. This concept is utflized in the Tandem Mirrors, which utilises the ambipolar potential of the plasma to create the required electrostatic potential. However at this point of time research along this line has been reduced drasticaily in the United States.

\section{Torotdal Confinement: The toroidal concept on the other hand} utilizes the fact that charged particles tend to follow the magnetic field lines. Thus an obvious suggestion is to design a ring shaped machine, so that plasma particles remain confined by following torotdal field 1 ines. This simple scheme does not work, as there are significant forces trying to expel plasma out of the torus. One simpie way to understand the origin of this force is the fact that plasma is a diamagnetic medium and thus it tends to move towards the region of weak magnetic fleld. If there are no currents in the torus and if the lines of force are purely toroldal, then the magnetic field is weaker at the larger radil and thus plasma feels a force tending to expel the plasma outwards.

It is possible to avoid this difficulty by introducing a 'poloidal field', in addition to the original toroidal field. This polotdal field 


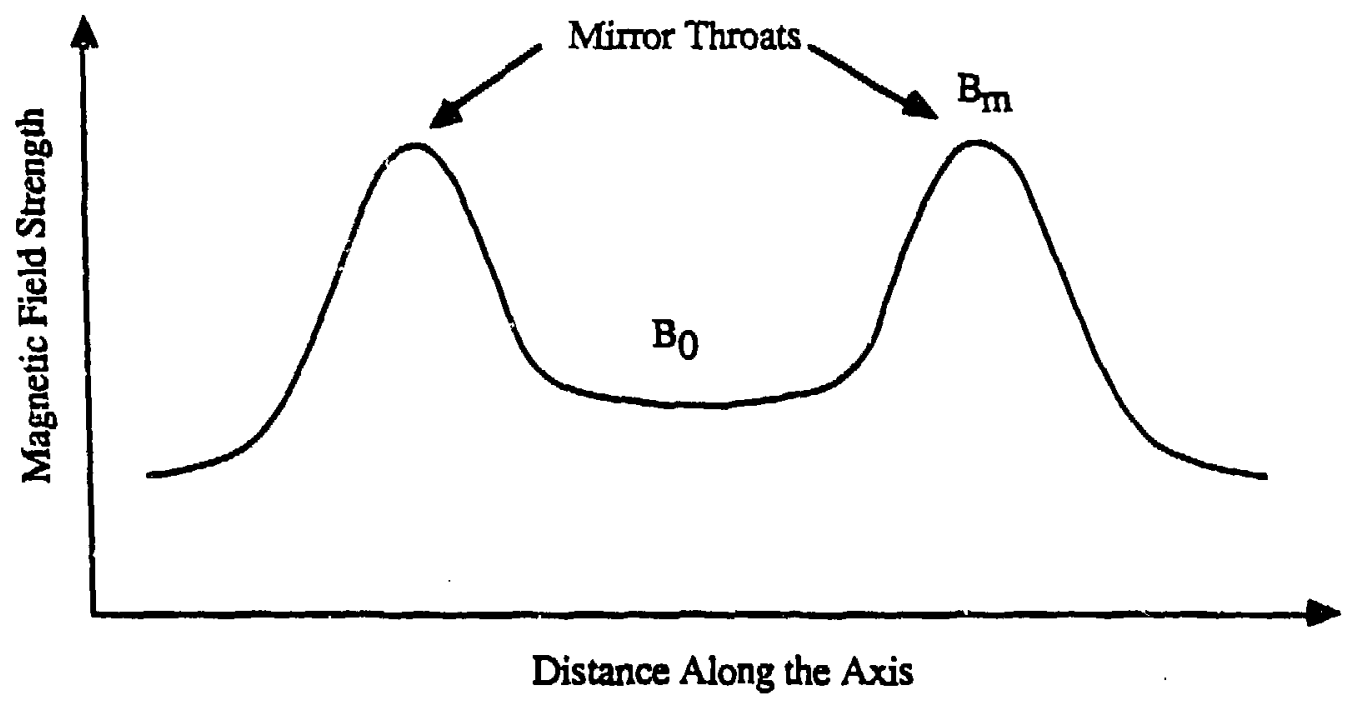

XBL 8610-3709

Fig. 1.1 
makes the field lines spiral around the torus leading to the creation of 'Rotational Transform', which makes plasma particles go around the torus at various radif and the net force tending to expel the plasma cancels out. Devices of torus-shaped controlled fusion machines differ from each other due to the ratio in the strength of the toroidal and poloidal magnetic fields, and also due to the distinction as to whether the poloidal fields are generated by toroidal currents outside or inside the volume occupted by the plasma.

There are two types of toroidal devices, which have been studied in greater detafl: Stellarator and Tokamak. These two differ in the way the poloidal component of magnetic field is generated. In stellarators, the pololdal component is generated by the currents in the external coll system; whereas in the tokamak, the poloidal component is generated by driving a toroldal current through the plasma itself.

Historically, the stellarator was the first concept to seem promising for fuston purposes. Significantly. there have been no ideas comparable in beauty and conceptual significance with that of the stelierator. The Idea behind this device is the general concept of toroldal magnetic configurations which is the basis of the theory of magnetic plasma confinement in toroidal systeils. It has engendered such important concepts as the magnetic surface, rotational transform and shear of magnetic field Itnes, the average magnetic well, the separatrix and island structure of magnetic surfaces, stochastization of magnetic fieid lines, the divertor, etc., and it has become an integral and fundamental part of the physics of high temperature plasmas [Spitzer (1958)]. Surprisingly, after two decades of disappotinting results which were 
associated with stellerator, there seems to have been turn around in the experimental findings. Very recently, mostly due to better theoretical understanding of toroldal confinement systems, the stellerartors seem to be achieving conditions approaching those of tokamaks [Yoshikawa (1985)].

However, the most promising candidate for the purpose of fusion seems to be the tokamak at this time. The essential difference between the tokamak and stellerator is the way in which the poloidal component of the confining magnetic field is generated. The stellerator uses currents in the external colls for this purpose, whereas the tokamak relies for magnetic confinement on longitudinal plasma currents induced with the help of a transformer type device. This difference however also makes the stellarator a steady state device, whereas tokamaks need to be run in a pulsed mode (However, there has been some progress towards achieving steady state operation of tokamaks). JET and TFTR, the two newest and largest tokamaks, are both nearing the Breakeven Point and they have achieved confinement times of about $1 / 2$ second with plasma densities approaching $5 \times 10^{23} \mathrm{~cm}^{-3}$ at the temperatures in the few $\mathrm{KeV}$ range. Howevar the pulsed nature of the device at this point creates many technical difficulties, which are due to extreme change in temperatures and other conditions reactors must withstand.

There are several other concepts of magnetic confinement along the two main lines mentioned above, but we would not consider them here. Instead at this point we would like to make a general comparison between the two type of devices: toroidal (closed) devices and Mirror (open) devices. (Here $B$ refers to the ratio of plasma pressure to that of magentic field energy density). 
Toroldal (closed)

- Low B

- Need to limit radial transport

- Pulsed operation in Toxamak

- Economic on large scale
Mirror (open)

- HIgh B

- Need to limit longitudinal loss

- Steady state operation

- Linear geometiry offers simpler design

From the comparison it is clear that Mirror devices have certain definite advantage over the toroidal devices. However, one is tempted to ask if there is a way to combine the good features of the two type of devices into a single one. That is, can one have a steady state device with high beta but no longitudinal loss.

In this thesis, we study some aspects of one such device, in which helical linking of mirrors is proposed by Kunkel (1982). In Chapter III, we describe the scheme of helically linked mirrors. We make a theoretica 1 analysis of this arrangement in Chapter IV, followed by an estimation of the maximum equilibrium $\beta$ value in chapter $V$. In chapter VI the condition for the vanishing of charge-neutralization currents and the theoretical basis for achieving this by rotating magnetic surfaces is presented. In Chapter VII, a computational analysis and its result is given. In Chapter VIII, some aspects of stabilizing the device is mentioned. In Chapter $[X$, we conclude with a summary. We would however first revtew some other studies made alang simtlar lines by other people in the next chapter. 
Chapter I I

Review of Toroidally Linked Mirror Configurations

The attempt to link mirrors together in a closed magnetic confinement device has beer made several times. Some of them have not been concluded fruitfully and some are still under investigation. We take a look at some of these attempts here. All of them have the same objective, namely, steady state operation combined with high beta and no longitudinal loss.

\section{Stellarator-Hirror Machine Target Plasma Reactor}

This concept was first mentioned in Dawson and Furth [Dawson et a?. (1971)j. The concept grew out of the so-called two-component tokamak scheme proposed earlier, as a beam-driven reactor. In this particular scheme a stellarator has a mirror machine section (See Fig. 2.1 and 2.2) so that the stellarator could hold relatively "cool" tritium ( $\leq 5 \mathrm{KeV}$ ) target plasma, whereas the mirror machine section would hold the energetic deuterons. In Fig. 2.1, the magnetic field strength and vartous components producing it are shown. In Fig. 2.2, the schematic diagram is shown. It is polnted out that there is a basic concern for the stabllity of an energetic loss-cone distribution immersed in the low temperature target plasma. They refer to a paper sy Guest et a1. (1970), Which made a general conclusion that large ratios of target density to energetic ion density are stabilizing, provided the ratio of the energetic ion "temperature" to the target fon temperature is not too great. It is stipulated that the target ion temperature required for 


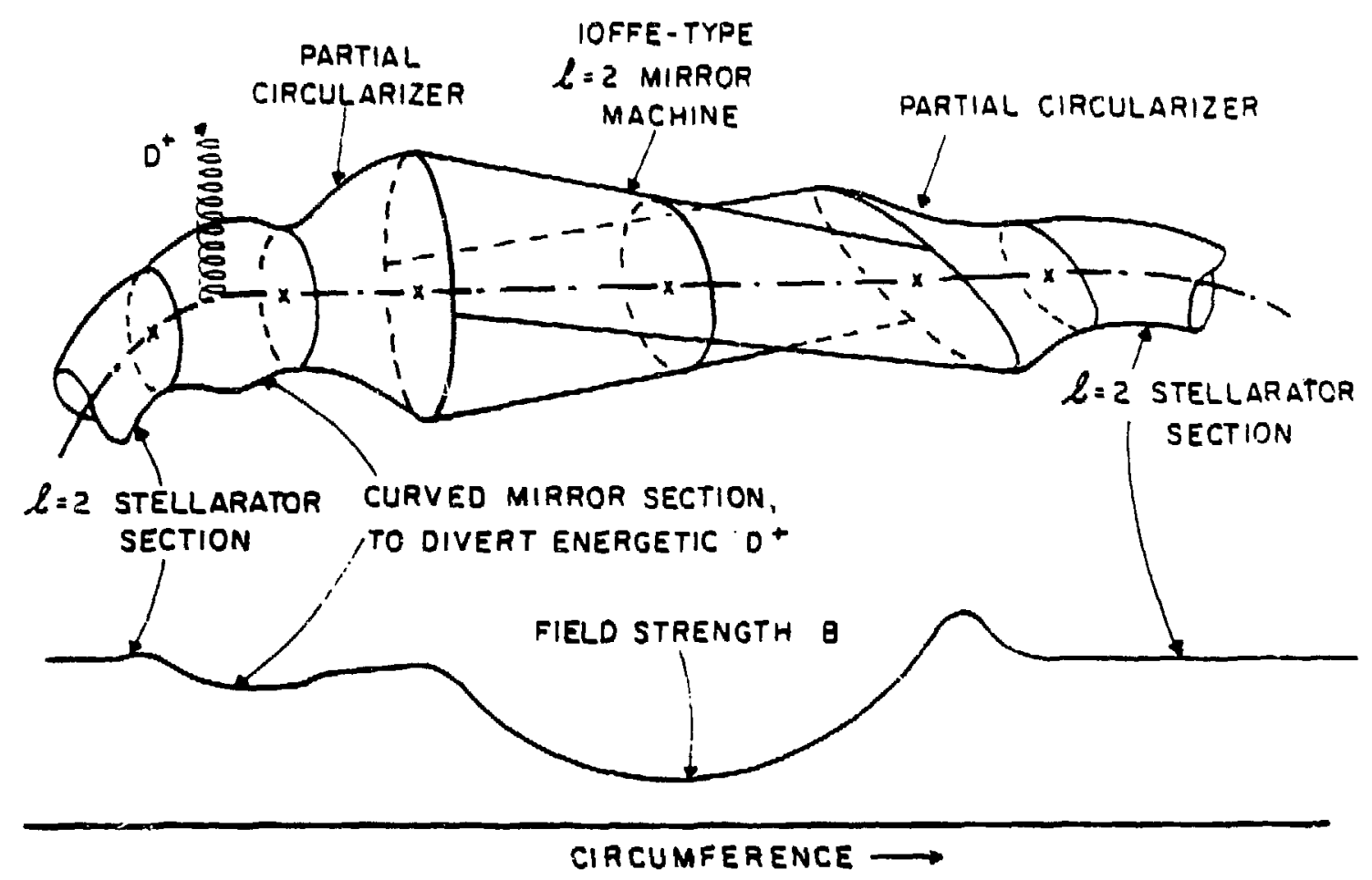

XBL $8610-3710$

Fig. 2.1 


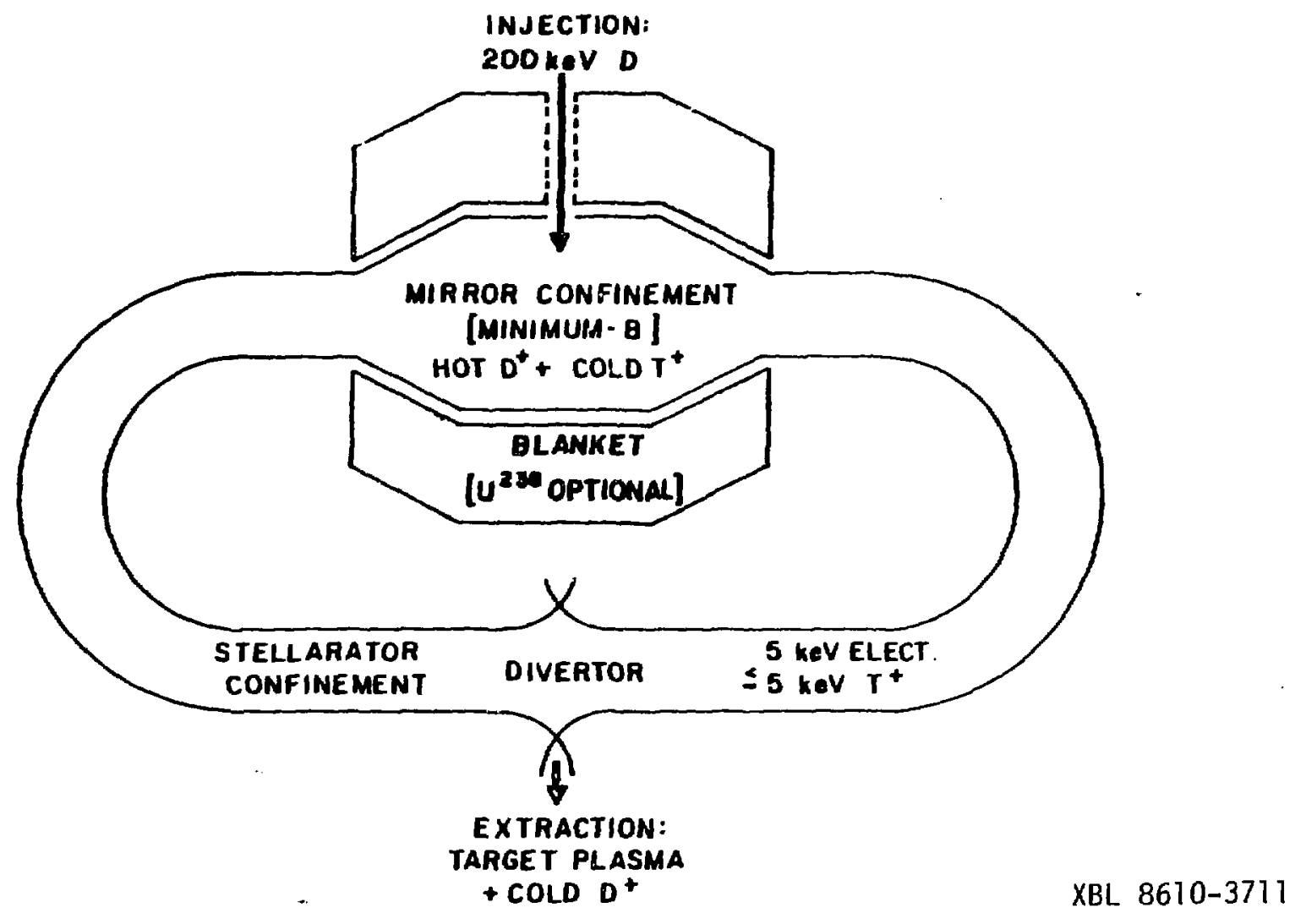

Fig. 2.2 
stabllity are somewhat lower than the electron temperatures required to give reasonable f-factors (ratio of nuclear energy release to initial deuteron energy).

Adyantages of this hybrid system with various other two-component devices are also discussed in the paper. Wtth respect to the standard stellarator the advantage of having blanket and neuteron shield extend over the mirror section only and thus avoiding the technical problem of putting helical coils outside the blanket, is mentioned. Also, the possiblity of smaller reactor size is pointed out. There is also the possibility of the minimum-B mirror section having strong favorable effect on low-frequency instabilities of the stellarator. With respect to the two-component tokamak the advantage of steady state operation of this hybrid scheme is pointed out. The conventent divertor operation and refuelling is brought to the reader's attention (See Fig. 2.2), and a comparison with respect to a Mirror Machine Reactor is also given. We could not find any further follow-up to this scheme in the literature.

\section{Torofdally Linked Mirrors}

Among the earlier attempts reported, on which a number of papers were published, was the work of Cordey and Watson (1974) of Cuiham Laboratory (U.K.). Their scheme envisaged toroldal linking of a relatively small number ( 8$)$ of minimum-B mirror fields, so that particles passing through the throat of one mirror necessarily pass into the adjacent mirror. In consequence the dominant particle loss mechanism becomes the escape through radial drift and enhanced radial diffusion of those particles which are reflected close to the mirror throats. This process 
is faster than the loss of particles from axisymetric tori by neoclassical radial diffusion, but slower than loss by free flow along the field lines. Since particles have a finite probability of being scattered by coulomb collisions out of the narrow 'loss disc' in velocity space within which this radial loss can take place, the resulting plasma distribution function includes a significant number of passing particles. The ratio of the pressures of the trapped and passing particles is a key parameter: if the ratio is too low, so that the pressure is essentially isotropic and nence constant along a flux tube, the maximum $B$ value is reduced to a few percent, as in a conventional steady-state toroidal contzinment system. Conversely if the ratio is as high as in a conventional mirror machine, altholigh the B limit is high, the energy containinent time drops to that of the conventional mirror and the advantage of linking is lost. Thus there is an optimum ratio of trapped to passing particle pressures. The authors report that preliminary calculations indicate that this is reached when the passing particle pressure is about $20 x$ of the total, and the energy containment time is then enhanced by a factor of about 5, with ifttle or no reduction in the maximum $B$ value below that of a conventional mirror. It has been indicated in the paper that the conditions necessary to achieve the optimum ratio are compatible with reactor requirements (densittes $-3 \times 10^{24}$; ton injection energy $\sim 100 \mathrm{keV}$; mirror magnetic field $\sim 100$ KG; radial dimension $\sim 1 \mathrm{~m}$ ). The loss of particles is localized in the neighborhood of the mirror throats, and it should be possible to destgn the magnetic field in such a way that the field lines just outside the separatrix at the throats guide the escaping particles into an energy recovery system. 
The authors have also provided some comparison with conventional mirror systems and with conventional toroidal reactors. They point out that in comparison to ordinary mirrors the TLM (Toroidally Linked Mirror) retains the advantage of steady-state operation, high $\beta$ value and easfer solution to the problems of heating, refuelling and diversion. They also argue that the principal engineering problems created by toroidal topology arise either because the space at the center of the torus is restricted(e.g. an iron core) or because helical windings are required which link the torus and prevent the withdrawal of sections of torus for repair or maintenance. They claim that the colls they were planning in their design are individually retractable and leave adequate space at the center of the torus. They also claim that the lower limit on the device size would not be as restrictive as that of tokamaks. It also removes the problem of low theoretical energy confinement time and the microinstabilities resulting from loss-cone plasma distribution functions. It is mentioned that a modular coll stellarator seems to be competitive with TLM reactor design. They have further studied some magnetic coll design in this paper.

Another paper deals with the plasma diffusion in the TLM system. [Cordey and watson (1975)]. In this they have examined the diffusion processes which determine the distribution function and containment time of a steady-state antsotropic toroidal plasma maintained by neutral injection in a confinement system conststing of a number of minimum- $B$ magnetic mirrors linked toroidally (1.e. TLM system). The theory of plasma diffusion in such systems depends upon the characteristics of single particle orbit in them. It has been shown that, in a TLM system, 
the particle drift surfaces are almost independent of energy but strongly dependent on the pitch angle (this is a situation which contrasts with that in toroidal confinement systems which possess magnetic surfaces). Both trapped and passing particles lie on closed and approximately superposable surfaces; however an intermediate class of particles with pitch angle lying in a "risk disc" have drift surfaces which are either closed but highly distorted or open. Otffusional plasma loss is typically due to repeated angular scattering into this risk disc, leading to loss by a random walk process in velocity space.

One more paper [Hastie and Watson (1977)] deals with ballooning instabilities in TLM systems. In the low-beta 1 imit at least TLM systems have been found to be stable against interchange modes (1.e. modes in which displacement $\boldsymbol{\sigma}$ is independent of distance along a fleld line $s$ ), provided that the plasma pressure is sufficiently anisotropic. However they are vulnerable to modes which are in some measure localized in $\mathrm{s}$, because such systems necessarlly have a finite plasma pressure in the linking regions (betweell the adjacent minimum- $B$ regions) where $\nabla p \cdot \nabla B$ is unfavorable. A formal expansion of the energy balance in powers of $B$ shows that, in lowest significant order, only interchange modes are allowed so that a study of these axial localized modes necessarily involves the inclusion of finite- $B$ effects. A general study of this has very little hope of analytital progress unless rather arbitrary simplifying assumptions are made. Ail TLM systems studied to date, the aspect ratio $\lambda=r_{0} / L$ (where $r_{0}$ is the radius of the plasma at a plane normal to the magnetic field passing through a field minimum and $L$ is the length between adjacent mirrors) is a small parameter - typically less 
than 1/ro. In this paper they include localized modes in the analysis. They claim that in cases of interest, quite high values of $B$ are obtained which are consistent with stability. We could not find any recent papers on this concept in the literature, which suggests that the concept was not followed to a fruitful conclusion.

\section{Linked 8aseball cotls}

The advent of twisted or modular colls opened the possibility for many new arrangements of torofdal confinement devices. One such concept was reported by Ohasa and Ikuta (1977). Their investigation was aimed at obtairing a linked Min-B configuration inside axially symmetric magnetic surfaces with large rotational transform by arranging the strongly twisted colls, 1.e., $\ell=m$ baseball colls, and at showing that the closed magnetic isobars are formed inside the last closed magnetic surface occupying a significant portion of the volume of the torus if $l=m$ baseball colls are chosen for the multipolarity of the colls. They report that the toroldal arrangement of $\ell=3$ baseball colls gives the desirable machine parameters, 1.e., large rotational transform and large volume of the last closed magnetic surface. They present some analytical and numerical study of the scheme. They conclude that closed isobars can be inside the high-shear magnetic surface. The mirror section could be useful for the contalnment of high-energy lons and the device can then be operated in a two-component mode.

\section{Dracon Confinement}

Several other papers dealing with linking of mirrors have been presented at conferences, but no details have been published in the open 
literature [Hall and McNamara (1974), Killeen et al. (1966)]. An interesting concept, is being followed by a group of scientists at the Kurchatov Institute of Atomic Energy in Moscow. They have proposed a concept called 'ORACON' confinement, which was first described at a European Conference in 1981 [Glagolev et al. (1981)]. The salfent point of the scheme is the design of a spectal connector of the Rectilinear Elements (CREL). The designing of CREL which links two straight sections is such that they have no effect on the equilibrium and transport in the straight sections. The requirement for this is that the charge-neutralization currents, which arise due to the curvature drift, should vanish in the straight section. Matheinatically, this leads to an integral condition being satisfied. We discuss this condition in detail later, in Chapier $y_{\mathrm{I}}$. One more advantage of this condition being satisfled is the fact that the equilibrium of the device does not depend on the length of the straight section in the first order. In this paper the authors mention some types of links which satisfy the required condition. They give an example of a planar axis CREL with nonuniform fleld, a planar axis CREL with uniform field and a CREL with a spatial (nonplanar) axis. It is shown that even a simple linking made out of three semicircular tori at the correct angle can satisfy this condition. This concept has been explored in various papers and is being followed further.

The ordinary methods of stabilizing the plasma in a Dracon have been considered in a separate publication [Arsenin et al. (1983)]. The use of quadrupole anchors at the end of straight sections to create an average magnetic well has been suggested, as well as use of RF waves to create 
ponderomotive stabilization of the stralght sections. A new method of stabilizing plasma in a Dracon by a combination of the curvature and the inhomogeneity of the magnetic field along the CREL axis is given in a later paper [Trubntkov and Glagolev (1984)], where also the shortest CREL for a homogeneous magnetic field is described. Plasma stabilization in a Oracon by triangular-elliptic deformation of the magnetic surfaces has also been calculated [Glagolev et al. (1984)]. We reproduce the treatment here in detail as this has implications for the device we have suggested in this thesis.

In this paper the authors considered both circular surfaces and surfaces with triangular deformation. A magnetic confinement device is said to have a magnetic well if the condition $V^{\prime \prime}(\Phi)<0$ holds. Here $\Phi$ is the longitudinal flux enclosed by a given magnetic surface and $v$ is the volume enclosed by the magnetic surface. It is shown that the expression for $V^{\prime \prime}(\Phi)$ reduces to the following for a DRAKON device satisfying the CREL condition.

$$
V^{\prime \prime}(\phi)=\frac{4}{\tau} \delta^{\tau / 2} L \frac{K d t}{B^{2} V\left(1-\varepsilon^{2}\right)}
$$

Here $t$ is a new dimensionless varlable, somewhat like a fictitous time, defined as

$$
t=\int_{0}^{s} K(s) d s
$$

and,

$$
\tau / 2=t(1 / 2) \text {. }
$$


The dimensioniess function $L$ is given as

$$
\begin{aligned}
L=1 & -\frac{9}{4} c+\frac{4 c^{2}}{1+c}+\frac{3}{4}\left(\frac{8}{B}\right)^{2}+\frac{1}{4} \dot{\eta}^{2}+(s \omega)^{2} \\
& -c \dot{n} \frac{B}{B} \frac{2 c Q_{0}}{1+c}-\frac{M_{0} B^{3}}{4 r\left(1-c^{2}\right)} \frac{d}{d t}\left(q_{2}^{2}+q_{2}^{2}\right) .
\end{aligned}
$$

where $c$ is the eccentricity and $n=\tanh ^{-3} c, \omega$ is the ratio of torston and curvature $x / k, q_{1}$ and $q_{2}$ are two integrals related to the geometric parameters of the device and other terms are defined in the paper. If there is no eccentricity $(c=0)$, we find that

$$
L_{c=0}=1+\frac{3 B^{2}}{4 B^{2}}-\frac{1}{4} B B^{2} \frac{d}{d t}\left(q_{1}^{2}+q_{2}^{2}\right)
$$

They mention that this case was studled by Mikhallovskil [Mikhallovskif (1983)], where it was shown that the magnetic well could be made positive If the magnetic surfaces were absolutely circular, but with a field nonuniform along the axis. The calculation for the case of surfaces with only elliptical (but no triangular) distortion yields a very weak magnetic well and the results are not given in the paper. However with triangular distortion they find that for mirror ratio of $2, \beta_{\mathrm{eq}}=12.2 \mathrm{X}$ and $B_{\text {stab }}=1.1 \%$. These values increase to $17.6 \%$ and $2.38 \%$ respesctively for a mirror ratio of 3 . However it is found that raising the mirror ratio also decreases the "working volume" Inside the separatrix. Thus one has to optimize between volume occupled by the separatrix and the B-values.

A new method of stabllizing the plasma by means of additional S-shaped mirrors in CRELs is suggested in a paper by Trubnikov et al. (1985). The destabilizing effect of the rectllinear parts has also been 
studied [Makurin and Mikhailovskif (1984)], and the effect of plasma self-stabilization by finite pressure can be found in a publication by Volkov et al. (1985). Finally, stabilization by means of quadrupole anchors has been considered in the past. [Whiteman et al. (1966), Solovev et al. (1967)]. The latest paper by Glagolov et al. (1985) gives a more comprehensive look at the search for a most suitable CREL. Various vartations of CREL are described in this paper. The effect of the length of straight sections on the plasma equflibrium in the Drakon device was discussed by Makurin et al. (1984). Inftially it was found that the length of the straight section does not effect the plasma equilibrium, by only considering terms linear in plasma pressure. However this study reports that there are restrictions on the length of the straight section If we take into account higher order terms. They find that the $B$-value in this case is given as

$$
B \approx B_{\max }\left(L_{c r} / L\right)^{2} \text {, }
$$

where $\beta_{\max }$ is the equitbrium $B$-value without taking into account the nonlinear terms, $L_{c r}$ is the length of the CREL and $L$ is the length of the straight section.

From looking at the various papers, which deal with toroldal linking of mirrors, there seems to be an increase of interest in this type of device. The most probable reason for this is a better theoretical understanding of Toroidal Confinement Devices. In the next Chapter we describe a scheme of helical linking of the mirrors, which is similar to the 'Dracon' concept but probably simpler. In this thesis we study this scheme in detafl. 
23

At this point, we would like to mention the advantage linked mirror schemes may have with respect to tandem - mirror arrangements [Logan (1983)]. The use of ambipolar potential barriers to reduce the end-loss in the mirror confined plasma has become complex and even then all the components put together do not seem to work as expected. The amount of energy input to maintain the ambipolar potential is quite high. We may expect to overcome these difficulties using a linked-mirror scheme. A final comparison can only be made, when the linked mirror scheme has been studied in greater detail. 


\section{Chapter I I}

\section{Description of Hellcally Linked Mirrors Scheme}

As seen earlier successful linking of mirrors into a closed configuration has certain distinct advantages over conventional magnetic confinement devices. We have also discussed some of the attempts being made for this purpose. The latest attempt at this, i.e. the Dracon device, has its links which are complex and from an experimental point of view may be difficult to construct. We therefore, decided to look at a simpler scheme of linking the mirrors by means of helical solenotds. In addition to being relatively simple to construct, this also has the advantages of having a high rotational transform due to torsion in the linking. In a sense, this configuration can be thought of as a type of stellarator or Heliac, the volume of which is greatly enlarged by insertion of straight axisymetric mirror sections. The latter can presumably operate at relatively high values of beta, consistent with the reduced field between mirrors, leading to an enhanced average $B$.

Due to the high rotational transform, confinement of passing particles is good. We think it is possible to use noncircular magnetic surfaces to achleve an average magnettc well and also have vanishingly small charge-neutralization currents flowing through the straight section. We describe these in more detall later on.

The device has to be nonplanar to make use of helical linking in a smooth way. The geometric axis of the device consists of alternating straight and helical segments. For the device to have n-straight segments we generate the helical axts by wrapping the straight line over 
a cylinder, whose axis is at an angle $\pi / n$ to the straight line. This makes the transition smooth up to first order. However, the curvature and the torsion are discontinuous at the transition point. The angle by which the straight line wraps around the cylinder is dependent upon the length of the straight segment desired and the radius of the generating cylinder. The torsion, and hence, the rotational transform is determined from these parameters. The details of the program used to create the coil data is given. in Appendix-c.

The selection of the number of segments is dependent upon various considerations. A full study of this and the optimization of parameters for a reactor type device or otherwise has not been undertaken as yet. However, as shown in Fig. 3.1, the number of segments we have chosen to study is the lowest possible, i.e. $n=3$. (The $n=2$ case is simply a racetrack configuration, which does not have any torsion and thus no rotational transform.) In the figure, the coils along with one generating cylinder is shown; ' $H$ '. ' $S$ ' and ' $G$ ' represent helical section, straight section and generating cylinder respectively. The device as shown in the figure is rotated by a small amount to show the helical nature of the coll axts distinctly. Dimensions are in centimeters. We should point out here that although the generating cylinder has been shown to be touching the coll perimeter for clarity of the picture, in realtty it is the geometric axts of the device that lies on the surface of the generating cylinder. We call the device THELMA (an acronym for Irtangular Heltcally Linked Mirror Arrangement).

The spacing of the colls to generate the desired field can be chosen in a way to have the smallest posstble ripple on the magnetic axts (say, 


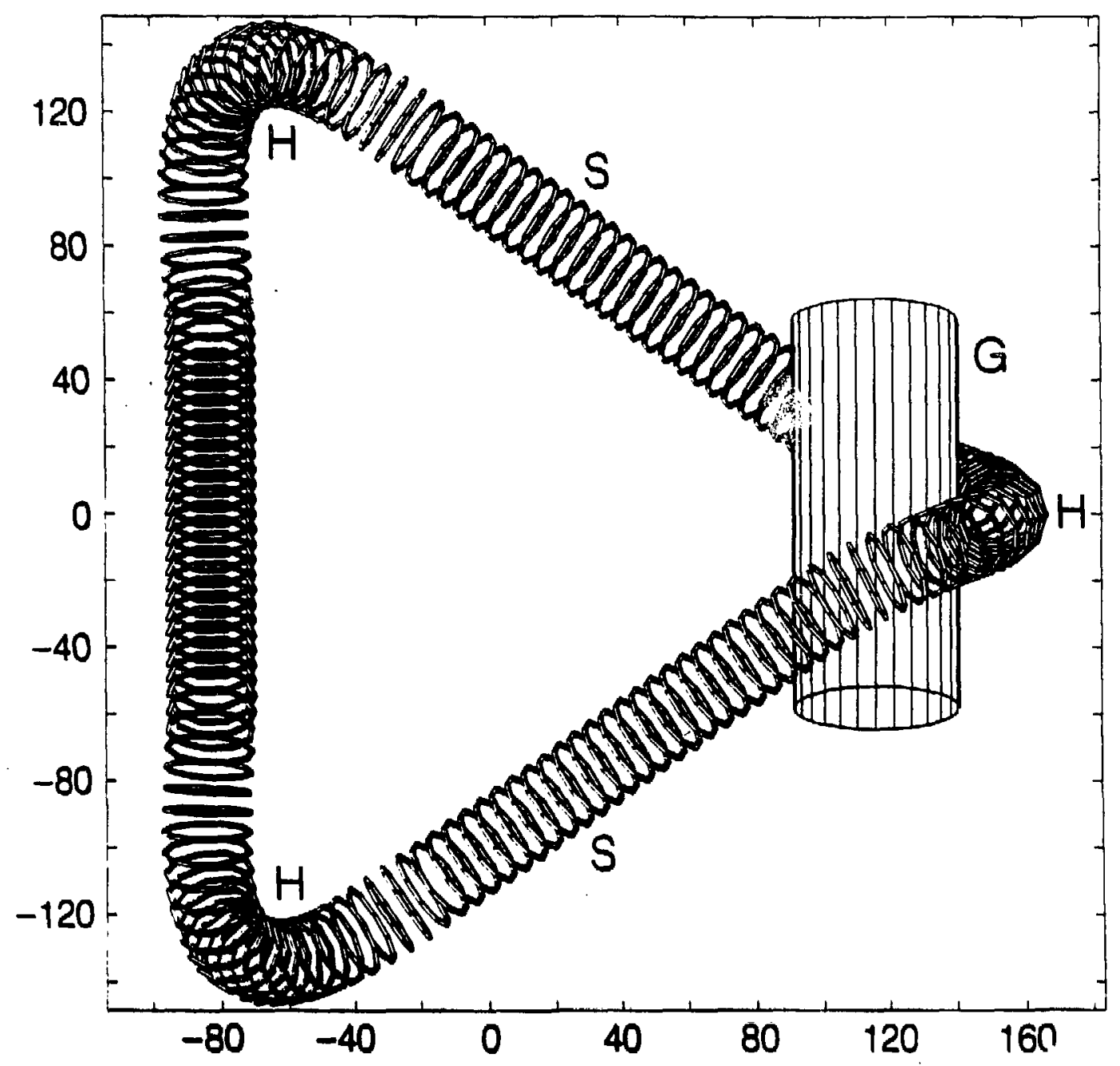

XBL 862.9744

Fig. 3.1 
less than 1x). In most cases, this means keeping the distance between the colls less than half the radius of the colls. In the helical sections the magnetic field does not vary much along the hellx but is, of course, a function of distance along the local radius of curvature. Particles with small velocity ratio $V_{\|} / V_{\perp}$ there will experience relatively large drift excurstons that depend on their position. Thus if the strongest flelds in the system are within, the bends, the trajectories of barely passing and of reflected particles in these reglons will not be axisymmetric and will give rise to neoclassical drift-orbit diffusion of the class of particles that enter the helical sections with very small parallel velocities. We decided to minimize this effect in the present system by the addition of strong axisymmetric mirror "throats" (or "bottlenecks") at the ends of each axisymmetric straight sections. The magnetic fields in the mirror throats $\left(B_{t}\right)$ of the axtsymetric straight sections must all be equal to each other and must be stronger than anywhere else on any given flux surface (see Fig. 3.2). In this way any particle within the loss-cone of any mirror section will pass through all mirrors until it is scattered into a trapped orbit. If a particle is trapped in a straight section it will carry out only axisymetric drift motions and hence its diffusion will not be subject to neoclassical effects until tt is detrapped and becomes a passing particle again. Only particles trapped in the helfcal bends will perform banana orbits and hence contribute to neoclassical diffuston, or worse, f.e., will drift out rapidly in a few bounces. Presumably, the number of particles so affected could be $11 \mathrm{mited}$ to a small fraction of the total number in the device. These considerations were presented by Abt and Kunkel (1983), but a thorough quantitative analysis has not been carried out (see App. B). 


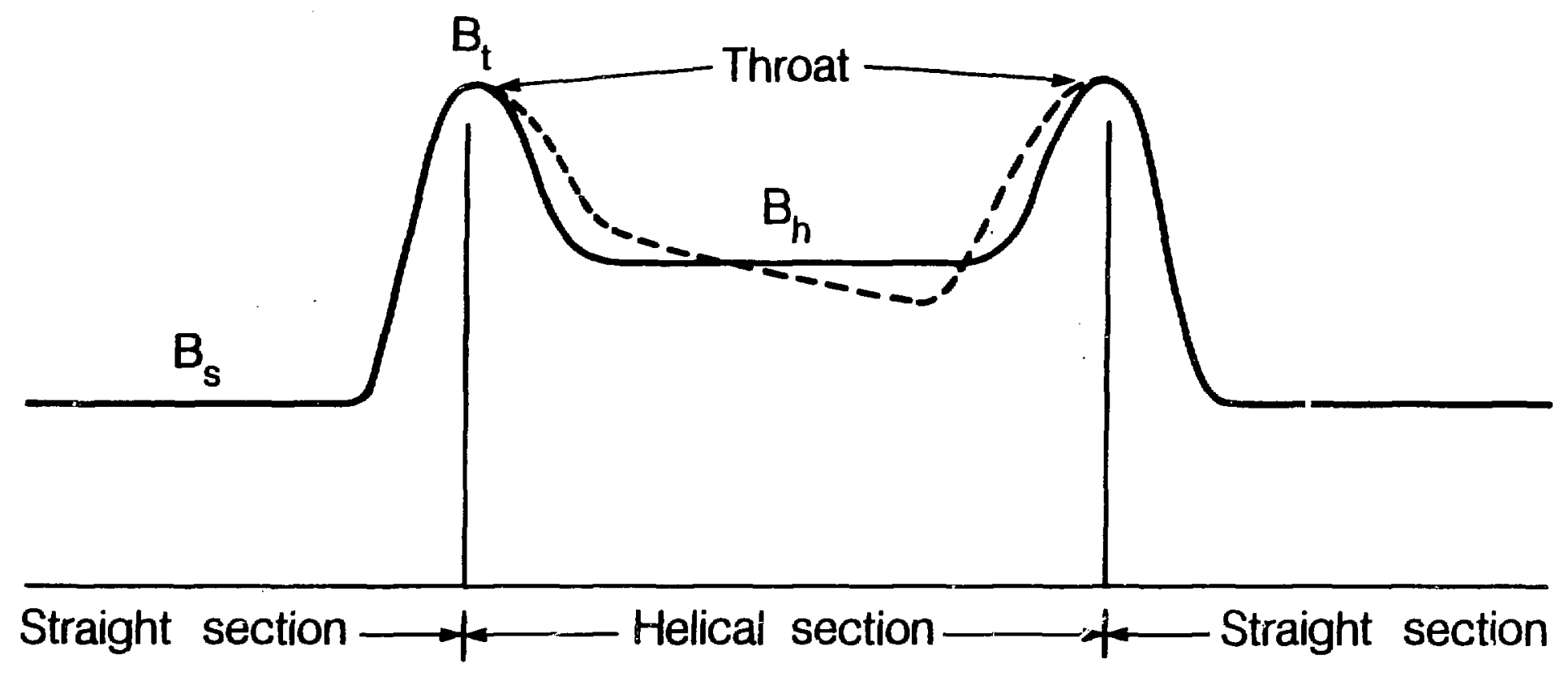

XBL $862-9735$

Fig. 3.2 


\section{Chapter IV}

\section{Theoretical Analysis}

In this chapter we obtain analytically an approximation to the magnetic field and then study single particle motion. For the analytical calculation of magnetic flelds we do not take into account the discontinuity in curvature and torsion at the junction of straight and helical sections. So, we treat the device as a solenofd and assume that the curvature is small. We use the Mercfer coordinate system as described in Appendix $A$. We follow the treatment outlined by Leontovich [Leontovich (1966), p.45] for this calculation. We start with the Laplace equation for a scalar potential $\phi$ associated with the magnetic field, which is given as-

$$
\nabla^{2} \phi=\frac{1}{\rho} \frac{\partial}{\partial \rho}\left(n_{s}^{\rho} \frac{\partial \phi}{\partial \rho}\right)+\frac{1}{\rho^{2}} \cdot \frac{\partial}{\partial \omega}\left(n_{s} \frac{\partial \phi}{\partial \omega}\right)+\frac{\partial}{\partial S}\left(n_{s} \frac{\partial \phi}{\partial s}\right)
$$

Here,

$$
n_{s}=1-k_{p} \cos \theta=1-k(s)_{p} \cos [\omega-a(s)] .
$$

In the zeroeth approximation (1.e. for a straight infinitely long solenotd) the magnetic potential inside and outside the solenotd are given by $\phi_{1}^{0}=B_{0} s, \phi_{\text {ext }}^{0}=0$, respectively. In this calculation we do not consider the mirror fleld in our device and thus we assume that in zeroeth approximation $B_{0}$ is independent of $s$. If we wanted to take into account the mirror fleld, we would modify this and consider $B_{0}$ to be an appropriate mirror magnetic fleld and follow similar steps in our calculation. However for our purposes, in which we are interested only in the nature of magnetic surfaces, we need not consider the mirror magnetic fleld, as this does not modify the magnetic surfaces 
qualitatively. The equation for the first approximation for the potential $\phi=\phi^{0}+\phi^{2}$ for a solenoid with a smooth axis is-

$$
\frac{\partial^{2} \phi^{2}}{\partial \rho^{2}}+\frac{1}{\rho} \cdot \frac{\partial \phi^{2}}{\partial \rho}+\frac{1}{\rho^{2}} \cdot \frac{\partial^{2} \phi^{2}}{\partial \omega^{2}}=-B_{0} \rho \frac{\partial}{\partial S}(k \cos \theta) \text {. }
$$

Where our assumption of small curvature allows us to expand the Laplace equation by keeping the terms which are linear in $\phi^{1}$. The solution of this equation is

$$
\begin{aligned}
& \phi_{1}^{1}=B_{0} s+\left(C_{1} \rho-\frac{B_{0}}{g} p^{3}\right) \frac{\partial}{\partial s}(k \cos \theta) ; \\
& \phi_{\text {ext }}^{2}=\frac{C_{2}}{\rho} \cdot \frac{\partial}{\partial 5}(k \cos \theta) .
\end{aligned}
$$

Assuming the solenoid to be an ideal conductor and using the requirement that the nomal component of the flelds must vanish at the surface of the solenoid $p=a$, we find

$$
c_{2}=0 ; C_{2}=\frac{3}{8} B_{0} \rho_{1}^{2} \text {. }
$$

From this it follows that the potential $\phi^{1}$ corresponds to a function of the magnetic surfaces which satisfies the equation $\nabla \Phi_{j} \cdot \nabla_{\psi}=0$, where

$$
\psi=\rho^{2}-\left(\frac{2 C}{B_{0}}-\frac{3}{4} \rho^{2}\right) k \rho \cos \theta \text {. }
$$

The displacement of the magnetic axts from the geometric axis is

$$
\Delta p=\frac{3}{8} \mathrm{ka}^{2}
$$

In the direction of the princtpal normal.

If we look at the terms carefully in eqn. (6), we expect the magnetic surfaces to have circular cross sections in the middle of the helical section. However the center and the amount of displacement decreases as we go away from the magnetic axis. Thus it may look somewhat as shown in Fig. 4.1. In the middle of the straight section we would expect 
Expected Magnetic Surface

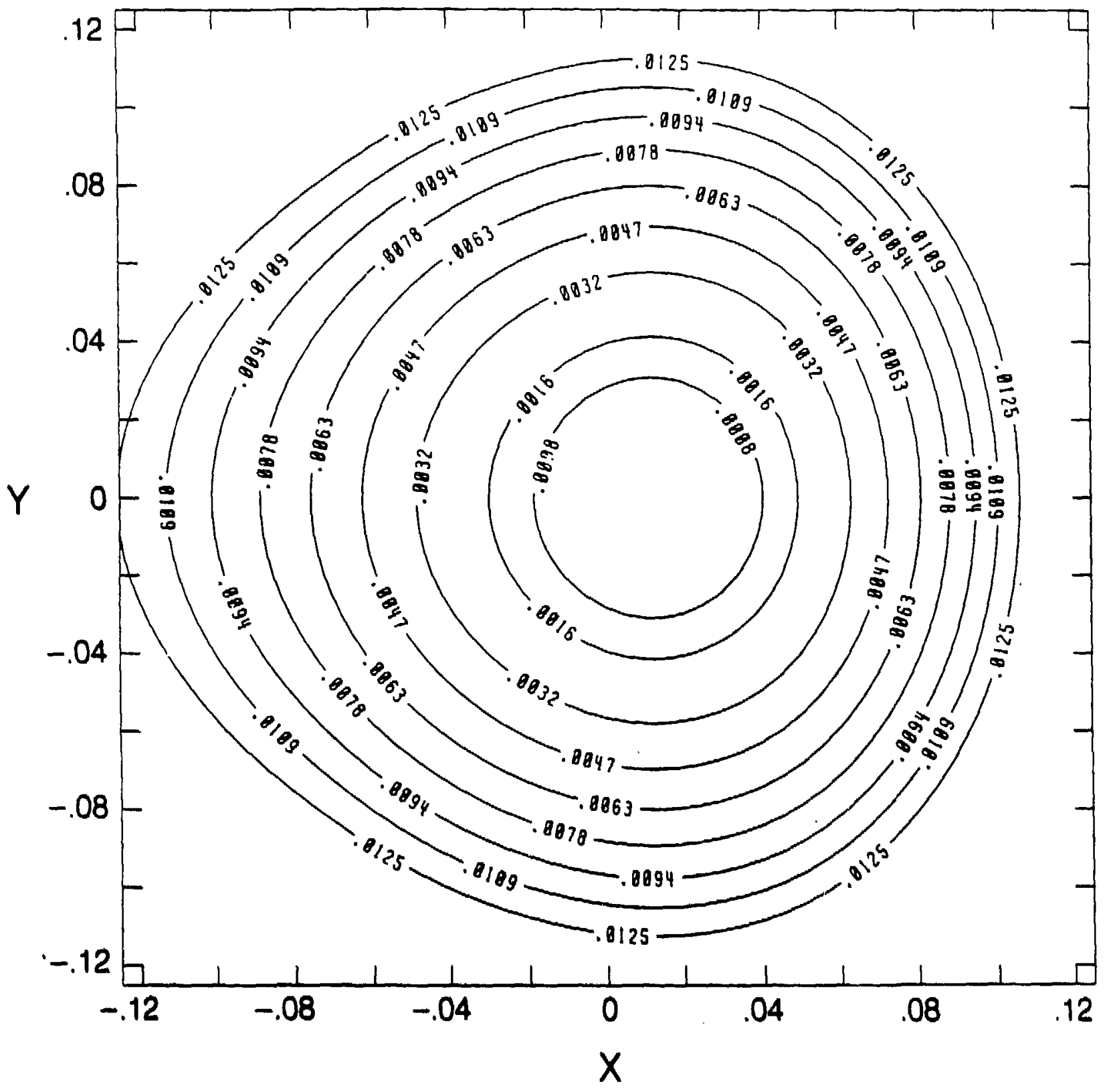

XBL 862.9746

Fig. 4.1 
concentric circular magnetic surfaces on the basis of this model, which may not be the actual case due to the discontinuous transition region. However one expects only a qualitative agreement with this model, which may be sufficient to understand the various numerical calculation results. Before proceeding further we would like to estimate the rotational transform of the field lines on the basis of this model, which is simply the transform provided by the torsion of the helical sections. Thus on the axis

$$
1=\int x d s
$$

Since torsion, $x=(\lambda / 2 \pi) /\left[(d / 2)^{2}+(\lambda / 2 \pi)^{2}\right]=$ const. for a constant pitch straight helix, where $\lambda$ is the pitch length, $d$ is the diameter of the generating cylinder. In our case $\lambda=-d / 2$ and each helical section has $2 / 3$ period, so that we get the total rotational transform as

$$
1=3 \cdot \frac{2}{3} \cdot \frac{2 r \lambda}{r\left(\lambda^{2}+\pi^{2} d^{2}\right)}=\frac{4 r}{\sqrt{5}} \simeq 322^{\circ}
$$

We expect only a small amount of shear to be present in the device with plane circular colls, since in this approximation the transform does not depend on $p$.

After this we would like to consider the drift orbits of single particles in this type of magnetic fleld conflguration on the basis of this model, again following Leontovich [(1966), p.207] and assuming adlabatic motion. The guiding center velocity including the curvature and the grad-B drift is given by 


$$
\begin{aligned}
& \frac{d \underline{r}}{d t}=v_{\|} \frac{B}{B}+\frac{m c\left(2 v_{\|}^{2}+v_{\perp}^{2}\right)}{2 e B^{2} v_{\|}}[\underline{B} \times \nabla B] ; \\
& =\frac{v_{\|}}{B}\left[\underline{B}+r_{g}\left(v_{a v}\right)(\hat{B} \times \nabla B)\right], \\
& v_{\perp}^{2} / B=\text { Const. } \\
& v_{\perp}^{2}+v_{\|}^{2}=v_{0}^{2}=\text { Const. }
\end{aligned}
$$

In the guiding center approximation the drift orbit surfaces, in such a system with magnetic surfaces, form modifted or "pseudo-magnetic surfaces" with the effective magnetic field given by

$$
\left.\underline{B}^{*}=\underline{B}+r_{g}\left(V_{a v}\right) \dot{B} \times \nabla B\right)=\underline{B}+\underline{B}_{1}
$$

This can be shown to be consistent with the drift equation (9) by expressing the latter in the form

$$
\frac{d \underline{r}}{d t}=\frac{V_{\|}}{B} \quad \underline{B}^{\star}=V_{\|} \frac{\underline{B}}{\left|B^{*}\right|} \text { as }\left|\underline{B}_{\mid}\right| \ll|B|
$$

In terms of the velocity ratio we express the effective magnetic fleld, whose fleld lines represent the drift orbits of particle guiding centers, as

$$
\begin{aligned}
\underline{B}^{*}=\underline{B} & +\frac{m c V_{0}}{e B^{2}} \frac{1+1 / 2 Y_{V}^{2}}{\left(1+1 / Y_{v}^{2}\right)}(\underline{B} \times \nabla B) \\
& \approx \underline{B}+\frac{m c V_{0}}{e B^{2}}\left(1+1 / 8 Y_{v}^{4}\right)(\underline{B} \times \nabla B)\left(\text { for } Y_{v} \equiv \frac{v_{1}}{V_{L}} \geq 1.4\right)
\end{aligned}
$$


From this we note that the perturbation term and hence the drift orbit deviation from the magnetic fleld lines-

(a) depends on sign of charge

(b) is insensitive to velocity ratio $\lambda_{v}>1.0$

(c) is proportional to the square root of the particle energy.

To estimate the deviation of the drift orbit surfaces from the magnetic surfaces we again follow Leontovich [(1966), p.207]. As discussed above the ensemble of trajectorles of guiding centers of particles with the same values of the integrals of motion, magnetic moment and energy, form a system of "magnetic" surfaces of the effective magnetic field

$$
\underline{B}^{\star}=\underline{B}+\underline{B}_{1}=\underline{B}+\frac{m c\left(2 V_{\|}^{2}+V_{\perp}^{2}\right)}{2 e B^{2} V_{\|}}[\underline{B} \times \nabla B]
$$

The equation for these surfaces $\psi^{*}=$ Const., which we shall call

drift surfaces, is determined from the condition $\frac{d \underline{r}}{d t} \cdot \nabla \psi^{\star}=0$ or

$$
B^{\star} \cdot \nabla_{\psi}^{\star}=0
$$

The supplementary term $\underline{B}_{2}$ in the expression for the magnetic fleld satisfles the condition

$$
\text { div } \underline{B}_{2}=0 \text {, }
$$

and the ratto of this term to $B$ is approximately the ratio of the gyro-radlus $r_{B}=v / \omega_{B}=m c v / e B$ computed for the total velocity to the radlus of curvature of the line of force $R$ :

$$
\left|B_{2}\right| /|B| \sim r_{B} / R
$$

Since it is assumed that $r_{B} / R \ll 1$, the correction can be regarded as a perturbation and the drift surfaces $\psi^{*}=$ const are subject to the same 
treatment that one uses in the case of perturbation of the magnetic surfaces [Leontovich (1966), p.209].

In the linear approximation in perturbation theory the deviation of the drift surface function $\Psi_{2}$

$$
\psi^{*}=\psi+\psi_{1} \text {, }
$$

from (16), the function satisfies the equation

$$
\underline{B} \cdot \nabla \Psi_{1}=-\underline{B}_{1} \cdot \nabla \psi \text {. }
$$

As in our model we have assumed that the magnetic fleld is constant on the magnetic axis in the zeroth approximation, $B_{0}=$ Const. Then, the basic contribution in the $\nabla B$ is given by the longitudinal field

$$
B_{s}=B_{0}\left[1+k(s)_{p} \cos \theta\right]
$$

The first terms in the expansion of the vector product $[8 \times \nabla B]$ and the velocity components $v_{\|}$and $v_{\perp}$ in serles in power of $p$ are then

$[\underline{B} \times \nabla B]_{\rho} \approx B_{0}^{2} k \sin \theta,[\underline{B} \times \nabla B]_{\omega} \approx B_{0}^{2} k \cos \theta$, $[\mathrm{B} \times \nabla B]_{s} \neq 0$.

$$
v_{1}^{2}=J_{1} B_{0}=\text { Const, } v_{\|}^{2}=v_{0}^{2}-J_{1} B_{0}=\text { Const. }
$$

In this case, the expression for $\underline{B}_{2}$ can be written

$$
\underline{B}_{2}=\frac{m c\left(2 v_{\|}^{2}+v_{\perp}^{2}\right)}{2 e B^{2} V_{\|}}[\underline{B} \times \nabla B]=\nabla_{\phi_{1}} \text {, }
$$

where

$$
\phi_{1}=\frac{m c\left(2 V_{\|}^{2}+V_{1}^{2}\right)}{2 e V_{\|}} k_{\rho} \sin \theta \equiv B_{2 y^{\rho}} \sin \theta .
$$

It is then apparent that the problem of finding drift surfaces reduces to the problem of determining the distortion in the magnetic surfaces caused by the effective perturbation of the transverse field $B_{2 y}$ 
directed along the binormal to the magnetic axis. As shown by Leontovich [Leontovich (1966), p.74)], the transverse field causes a displacement of the torotdal surfaces and some modification of their shape. The displacement of the axis is approximately $x_{0} \sim B_{2 y} L / B_{O_{1}}$. Substituting the value of $B_{1 y}$ from Eq. (24) we estimate the displacement of the drift axis with respect to the magnetic axis:

$$
x_{0}-\frac{V_{\Perp}+v_{\perp}^{2} / 2 V_{\Perp}}{\omega_{B}} \cdot \frac{K L}{1}
$$

Thus in the case when $K L / 1 \sim 1$, we expect the displacement to be of the order of radius of gyration of a particle with speed defind as $v_{a v}$. This condtion is satisfied in our case and hence we would expect that since the field lines are confined in THELMA, the single particle motion would be confined for particles satisfying the earlier pitch angle condition. We study this numerically in a later chapter. 


\section{Chapter $V$}

Estimate of the Maximum Equilibrium B

In this chapter we describe the method used to numerically estimate an upper limit for the equilibrium beta value. The plasma column has a transverse displacement due to the curvature of the toroidal device, which depends on the $B$ value of the plasma. However this transverse displacement is 1 imited by the transverse dimension of the device and thus gives an upper $1 \mathrm{imit}$ on the equilibrium beta value of the plasma in the device.

As earlfer, we use the Mercter coordinate system for this calculation (see Appendix-A). For this calculation we follow the treatment of Leontovich [(1966), p.135], however our system has no longitudinal current and thus we set the w-component of the magnetic field, $B_{\text {wo }}=0$. The starting equations are the equilibrium equations

$$
\begin{aligned}
& \frac{I}{c}[1 \times \underline{B}]=\nabla D ; \\
& \frac{(4 \pi)}{c} \perp=\operatorname{curl} B \\
& \text { div } \underline{B}=0
\end{aligned}
$$

From (1) we have the equivalent equations

$$
\begin{aligned}
& \underline{B} \cdot \nabla_{p}=0 \\
& \perp \cdot \nabla p=0
\end{aligned}
$$


We write the eqn. (1)-(3) in terms of their vector components. However we use the equivalent eqn. (4) \& (5) for writing the $\omega$ \& 5 components of eqn. (1). This gives us

$$
\begin{aligned}
& \frac{\partial p}{\partial p}=\frac{1}{c}\left(J_{\omega} B_{s}-J_{s}{ }_{\omega}\right) \text {; } \\
& B_{\rho} \frac{\partial p}{\partial \rho}+\frac{B_{\omega}}{p} \cdot \frac{\partial p}{\partial \omega}+B_{s} \frac{1}{T-k \rho \cos \theta} \frac{\partial p}{\partial s}=0 ; \\
& J_{\rho} \frac{\partial p}{\partial \rho}+\frac{J_{\omega}}{\rho} \cdot \frac{\partial p}{\partial \omega}+J_{s} \frac{1}{T-k \rho \cos \theta} \frac{\partial p}{\partial s}=0 \text {; } \\
& \frac{4 \pi}{C} J_{\rho}=\frac{1}{\Gamma-\frac{1}{\rho} \cos \theta}\left\{\frac{1}{\rho} \cdot \frac{\partial}{\partial \omega}\left[\left(1-k_{\rho} \cos \theta\right) B_{S}\right]-\frac{\partial B_{\omega}}{\partial S}\right\} ; \\
& \frac{4 \pi}{c} J_{\omega}=\frac{1}{\Gamma-k_{\rho} \cos \theta}\left\{\frac{\partial \theta}{\partial s}-\frac{\partial}{\partial \rho}\left[\left(1-k_{\rho} \cos \theta\right) \theta_{S}\right]\right\} \text {; } \\
& \frac{4 \pi}{c} J_{S}=\frac{1}{\rho} \cdot \frac{\partial}{\partial \rho}\left(\rho B_{\omega}\right)-\frac{1}{\rho} \cdot \frac{\partial B}{\partial \omega} \text {; } \\
& \text { का } \frac{1}{k \rho \cos \theta)}\left\{\frac{\partial}{\partial \rho}\left[\left(\rho-k_{\rho}^{2} \cos \theta\right) B_{\rho}\right]\right. \\
& \left.+\frac{\partial}{\partial \omega}\left[\left(1-k_{\rho} \cos \theta\right) B_{\omega}\right]+\rho \frac{\partial B_{S}}{\partial s}\right\}=0 \text {. }
\end{aligned}
$$

Now we make the assumption that the curvature $k$ is small so that we can make an expansion in $k_{p}$, assuming it to be a small parameter. So we write

$$
\begin{aligned}
& p=p_{0}(\rho)+p_{2}(\rho, \omega, s) \\
& \underline{B}=\underline{B}_{0}(\rho)+\underline{B}_{1}(\rho, \omega, s) \\
& \perp=\mathbb{1}_{0}(\rho)+\underline{I}_{2}(\rho, \omega, s)
\end{aligned}
$$

where

$$
p_{0}(p) \text {; }
$$




$$
\begin{aligned}
& \underline{B}_{0}(p)=B_{S 0}(p) \hat{e}_{s} ; \\
& I_{0}(p)=J_{\omega 0}(p) \hat{e}_{\omega}+J_{S 0}(p) \dot{e}_{S} .
\end{aligned}
$$

is the solution of the equilibrium equation in the zeroth approximation, $k(s)=0$. The zeroth approximation corresponds to a cylinderically symmecric distribution and, as is well known, the distribution is charactetrized by the following pressure balance equation:

$$
8 \pi\left[\left\langle p_{0}(p)\right\rangle-p_{0}(b)\right]+\left\langle B_{S 0}^{2}(p)\right\rangle=B_{S 0}^{2}(b)+B_{\omega 0}^{2}(b)
$$

where

b is the outer radius of plasma column, and

$$
\begin{aligned}
& \langle P(p)\rangle=\frac{1}{\pi b^{2}} \int_{0}^{p} P_{0}(p) 2 \pi p d p ; \\
& \left\langle B_{50}^{2}(p)\right\rangle=\frac{1}{b^{2}} \int_{0}^{p} B_{50}^{2}(p) 2 \pi p d p ;
\end{aligned}
$$

We expand the corrections assoctated with the curvature in a fourter expanstion as follows:

$$
\begin{aligned}
& p_{1}(p, \omega, s)=\operatorname{Re} \sum_{n} p_{n}(p) \exp \left[1\left(\omega-x_{n s}\right)\right] \\
& B_{1}(p, \omega, s)=\operatorname{Re} \sum_{n} \theta_{n}(p) \exp \left[1\left(\omega-x_{n s}\right)\right] \\
& J_{2}(p, \omega, s)=\operatorname{Re} \sum_{n} J_{n}(p) \exp \left[1\left(\omega-x_{n s}\right)\right] \\
& \text { Here, } \\
& x_{n}=1 / \int_{0}^{s} x d s-2 \pi n / L .
\end{aligned}
$$

As discussed earlier, in the first approximation in the expansion in the curvature, the cross sections of the magnetic surfaces remain circles 
40

but with displaced centers. The equation of the magnetic surface with a cross sectional radius $p^{\prime}$, at which the plasma pressure is $p\left(p^{\prime}\right)$, can be written in the following form in the first approximation:

$$
\rho^{\prime}=\rho+\xi(\rho, \omega, s)=\rho+\operatorname{Re} \sum_{n} \xi_{n}(\rho) \exp \left[j\left(\omega-x_{n} s\right)\right]
$$

By definition, the plasma pressure on this surface satisfies

$$
P(\rho+\xi, \omega, s)=P_{0}(\rho)
$$

Expanding the left side of this equation.

$$
P(p+\xi, \omega, s)=p_{0}(p)+p_{1}(p, \omega, s)+\xi(\rho, \omega, s) d p_{0} / d \rho+\ldots
$$

From this we get the relation between the displacement and the pressure correction in the linear approximation:

$$
p_{2}(\rho, \omega, s)=-E(p, \omega, s) d p_{0} / d p
$$

Now linearizing eds. (6)-(12), we obtain a system of eds. for the Fourier components of the pressure, the magnetic field, the current density, and the displacement:

$$
\begin{aligned}
& \frac{d P_{n}}{d p}=\frac{1}{c}\left(J_{\omega 0} B_{s n}-J_{s 0^{8}}{ }_{\omega n}+J_{\omega n} B_{s o}\right): \\
& B_{\rho n}=-1 \frac{E_{n}}{\rho}\left(x_{n} \rho B_{s o}\right): \\
& J_{\rho n}=1^{E} \frac{n}{\rho}\left(J_{w 0}-x_{n} \rho J_{s 0}\right) ; \\
& x_{n} \rho B_{w n}+B_{s n}=k_{n} \rho B_{s o}+\xi_{n}\left(J_{\omega 0}-x_{n} \rho B j_{s 0}\right) 4 \pi / c ; \\
& J_{\omega n}-x_{n} \rho J_{s n}=k_{n} \rho J_{\omega 0}-\frac{d}{d \rho}\left[\xi_{n}\left(J_{\omega O}-x_{n} \rho J_{s 0}\right)\right] ; \\
& \frac{4}{c} J_{s n}=\frac{1}{\rho} \cdot \frac{d\left(\rho B_{\omega n}\right)}{d \rho}-\frac{1}{\rho} B_{\rho n} ;
\end{aligned}
$$




$$
B_{w n}-x_{n} \rho B_{s n}=-\frac{d}{d p}\left[\xi_{n}\left(-x_{n} \rho B_{s o}\right)\right]
$$

In obtaining eqn. (33) we have replaced eq.(10) by the equation div $1=0$

Also, $K_{n}=\frac{1}{L} \int_{0}^{1} K(s) e^{-12 \pi n s / L} d s$.

Using eqs. (29), (32), (33) and (35) we can establish a relation between the magnetic field components $B_{w n}$ and $B_{\text {sn }}$ and the current density component $j_{\omega n}$ and $j_{s n}$ and the displacement $\xi_{n}$, and the derivative of the displacement $d \xi_{n} / d p$. Replacing these relation in (34) we obtain the following differential eqn. for the displacement:

$$
\begin{aligned}
& \frac{1}{\rho} \cdot \frac{d}{d \rho}\left\{\rho \frac{\left(x_{n \rho}{ }^{8} s o\right)^{2}}{1+x_{n}^{2} \rho^{2}} \cdot \frac{d \xi_{n}}{d \rho}\right\} \\
& -\frac{x_{n}^{2} \xi_{n}-k_{n}}{1+x_{n}^{2} \rho^{2}}\left\{2 \frac{x_{n}^{2} \rho^{2} B_{s 0}^{2}}{1+x_{n}^{2} \rho^{2}}+\left(x_{n} \rho B_{s 0}\right)^{2}+8 \tau p \frac{d p_{0}}{d \rho}\right\}=0 .
\end{aligned}
$$

As is clear the particular solution of the inhomogeneous equation is extremely simple: $\xi_{n}=k_{n} / x_{n}^{2}$.

If the curvature and the turning angle $a(s)=\int_{0}^{5} x d s$ are smooth

functions of the arc length $s$, the coefficlents in the expansion of the relative curvature $k_{n}$ fall off rapldly with index so that in the Fourier expansion, of al1 the toroldal corrections, the basic contribution comes from terms characterized by low values of $n$. For our case the curvature and torsion are discontinuous at the transition section. However in a real device this would not be the case. Thus we approximate the curvature and the torsion by the sus of a Fourter expanstion in which we 
keep terms only up to $n=7$. As can be seen in Fig. 5.1 (where the solid curve is the actual case and dashed curve is the approximation), we find that this approximation is quite close and under this approximation the following condition is satisfled:

$$
\left|x_{n} \rho\right| \ll 1 \text {. }
$$

Under these conditions we can omit the term $x_{n} F_{n}$ in eq. (37): then, integrating this equation once, we have

$$
\begin{aligned}
& \frac{d \xi_{n}}{d \rho}=k_{n} \rho \frac{G}{D}: \\
& G=8 \pi\left[<\rho_{0}>\rho-p_{0}(\rho)\right]+\frac{1}{2}\left[-3\left\langle x_{n}^{2} \rho^{2} B_{s 0}^{2}>\rho\right] ;\right. \\
& D=\left[x_{n} \rho B_{s o}(\rho)\right]^{2} .
\end{aligned}
$$

The Fourier components of the ralative displacement of the magnetic surfaces with cross-sectional radif $b$ and $b^{\prime}$ are then given by

$$
E_{n}=k_{n} \int_{b}^{b^{\prime}} \rho \frac{G}{D} d \rho
$$

In these formulas, $\left\langle p_{0}\right\rangle_{p}$ etc, are mean values taken over the cross-sectional radtus $\rho$

$$
\left\langle P_{0}>p=\frac{1}{\pi \rho^{2}} \int_{0}^{\rho} P_{0}(\rho) 2 \pi \rho d \rho=\frac{1}{\pi \rho^{2}} \int_{0}^{2 \pi} d \omega \int_{0}^{\rho} p(\rho, \omega, s) \rho d \rho\right.
$$

etc.

We can use eq. (40) to compute the displacement of the magnetic surface of radius $p$ with respect to the plasma of radius $b$. By the plasma radius a we are to understand the radius of the cross section of some outermost magnetic surface beyond which the current density is 
Approximation to Curvature

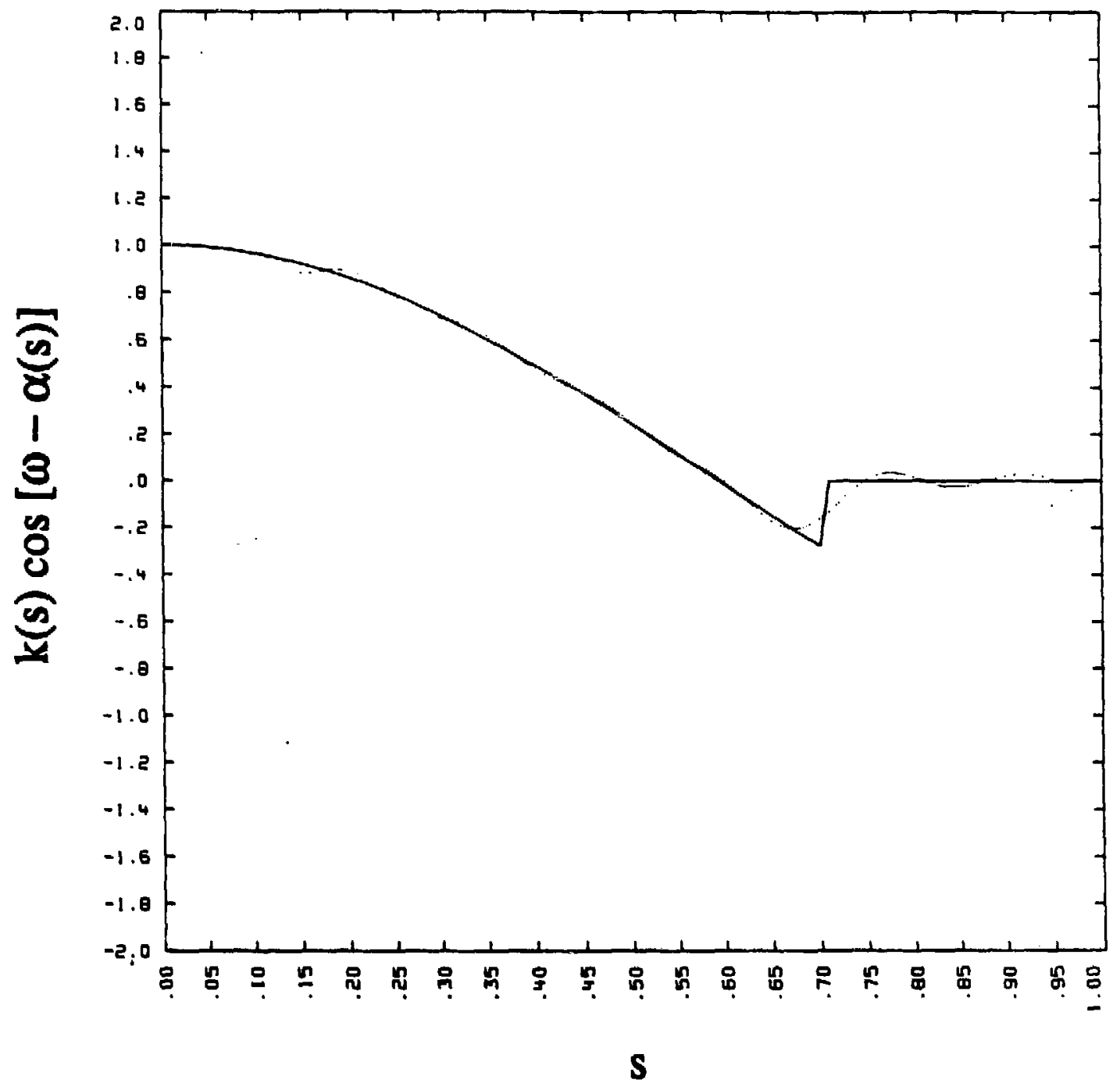

XBL $8610-3712$

Fig. 5.1 
neglegibly small. Then outside the plasma $(\rho \geq b)$ the mean values that appear in (40) can be expressed in the form

$$
\begin{aligned}
&\left\langle p_{0}\right\rangle_{p}=\left\langle p_{0}\right\rangle_{b} b^{2} / \rho^{2} ; \\
&\left\langle x_{n}^{2} \rho^{2} B_{S O}^{2}\right\rangle p= x_{n}^{2} B_{S O}^{2}(b) \rho^{2} / 2+ \\
&\left\{\left\langle x_{n}^{2} \rho^{2} B_{S O}^{2}\right\rangle_{b}-x_{n}^{2} B_{S O}^{2}(b) b^{2} / 2\right\} b^{2} / \rho^{2} .
\end{aligned}
$$

The last term is of the order of $x_{n}^{2} \rho^{2}\left(B_{\omega}^{2} / 2+4 \pi p\right)$, and can be seen using Eq. (19). We neglect this term. Carrying out the integration we obtain that

$$
\xi_{n}=-\frac{3}{B} k_{n} \rho^{2}\left(1-\frac{b^{2}}{\rho^{2}}\right)+\frac{k_{n} \beta}{2 x_{n}^{2}}\left(1-\frac{b^{2}}{\rho^{2}}\right) .
$$

The first term here determines the displacement of the vacuum magnetic surfaces, the second term determines the displacement of the magnetic surfaces associated with the plasma pressure. In the device under consideration,

$$
\begin{array}{rlrl}
k(s) & =k_{0} & & \text { for } 0 \leq s \leq 1_{c} \\
& =0 & & \text { for } 1_{c}<s \leq 1_{c}+1_{s}=L \\
x(s) & =x_{c} & & \text { for } 0 \leq s \leq 1_{c} \\
& =0 & \text { for } I_{c}<s \leq 1_{c}+1_{s}:
\end{array}
$$

So that, $x_{n}=x_{c}{ }^{1} c / L-2 \pi n / L \quad$ (46)

$$
\text { and } \begin{aligned}
k_{n} & =\frac{k}{L} \int e^{t\left[x_{0} s-x_{c} s-2 \pi n s / L\right]} d s \\
& =\frac{k}{L} \int e^{t\left[x_{n}-x_{c}\right] s} d s
\end{aligned}
$$


Hence, Re $\left.k_{n}=k \sin \left\{\left(x_{n}-x_{c}\right)\right]_{c}\right\} /\left\{L\left(x_{n}-x_{c}\right)\right\}$.

and, $\left.\operatorname{Im} k_{n}=2 k\left[\sin \left\{\left(x_{n}-x_{c}\right)\right]_{c} / 2\right\}\right]^{2} /\left\{L\left(x_{n}-x_{c}\right)\right\}$

Now, since by defintition,

$$
\begin{aligned}
& \xi\left(p^{\prime}, \omega, s\right)=\operatorname{Re}\left[\xi_{n}\left(p^{\prime}\right) \exp \left[i\left(\omega-x_{n} s\right)\right],\right. \\
& =\varepsilon\left[\left(\operatorname{Re} \xi_{n}\right) \operatorname{Cos}\left(\omega-x_{n} s\right)-\left(\operatorname{Im} \xi_{n}\right) \sin \left(\omega-x_{n} s\right)\right]
\end{aligned}
$$

From(43).

$$
\operatorname{Re} \xi_{n}=\operatorname{Re} k_{n}\left[-(3 / 8) \rho^{2}\left(1-b^{2} / \rho^{2}\right)+B\left(1-b^{2} / \rho^{2}\right) / 2 x_{n}^{2}\right] \text {. }
$$

and $\operatorname{Im} \xi_{n}=\operatorname{Im} k_{n}\left[-(3 / 8) \rho^{2}\left(1-b^{2} / \rho^{2}\right)+B\left(1-b^{2} / \rho^{2}\right) / 2 x_{n}^{2}\right]$,

So that,

$$
\begin{gathered}
\xi\left(\rho^{1}, \omega, s\right)=\left[-(3 / 8) \rho^{2}\left(1-b^{2} / \rho^{2}\right)+B\left(1-b^{2} / \rho^{2}\right) / 2 x_{n}^{2}\right] \times \\
\times \quad \sum\left[\left(\operatorname{Re} k_{n}\right) \operatorname{Cos}\left(\omega-x_{n} s\right)-\left(I m k_{n}\right) \sin \left(\omega-x_{n} s\right)\right\}
\end{gathered}
$$

Thus we see that the displacement $\xi$ depends linearly on $B$. To get an idea of what limit this imposes on the maximum value of a obtainable, we consider a case in which the plasma radius is half the radius of the solenoid. We show in Fig. 5.2 a plot of the maximum displacement of the plasma boundary as a function of the $B$ value. We have shown three different cases of solenold radius and find that a larger radius leads to a larger value of maximum equilibrium $B$. For the case of $R=12.5 \mathrm{~cm}$., we find the maximum $B$ to be $-5.5 \times$ as shown by the dashed line. We cannot use this analysis for calculating a maximum $B$ value for much larger values of $R$ with the same value of $k$ without violating the approximation under which the calculation was done. However these numbers are only estimates and should be used for order of magnitude calculations only. In the theoretical treatment no mirror magnetic fleld in the straight 
section is assumed. But, we may stipulate that with a mirror ratio of 4 , the maximum $\beta$ value in the straight section could be $\sim 20 x$.

Thus we find that even with unoptimized parameters, the device is capable of moderately high $\beta$ values. This should be extremely useful for economic purposes. 


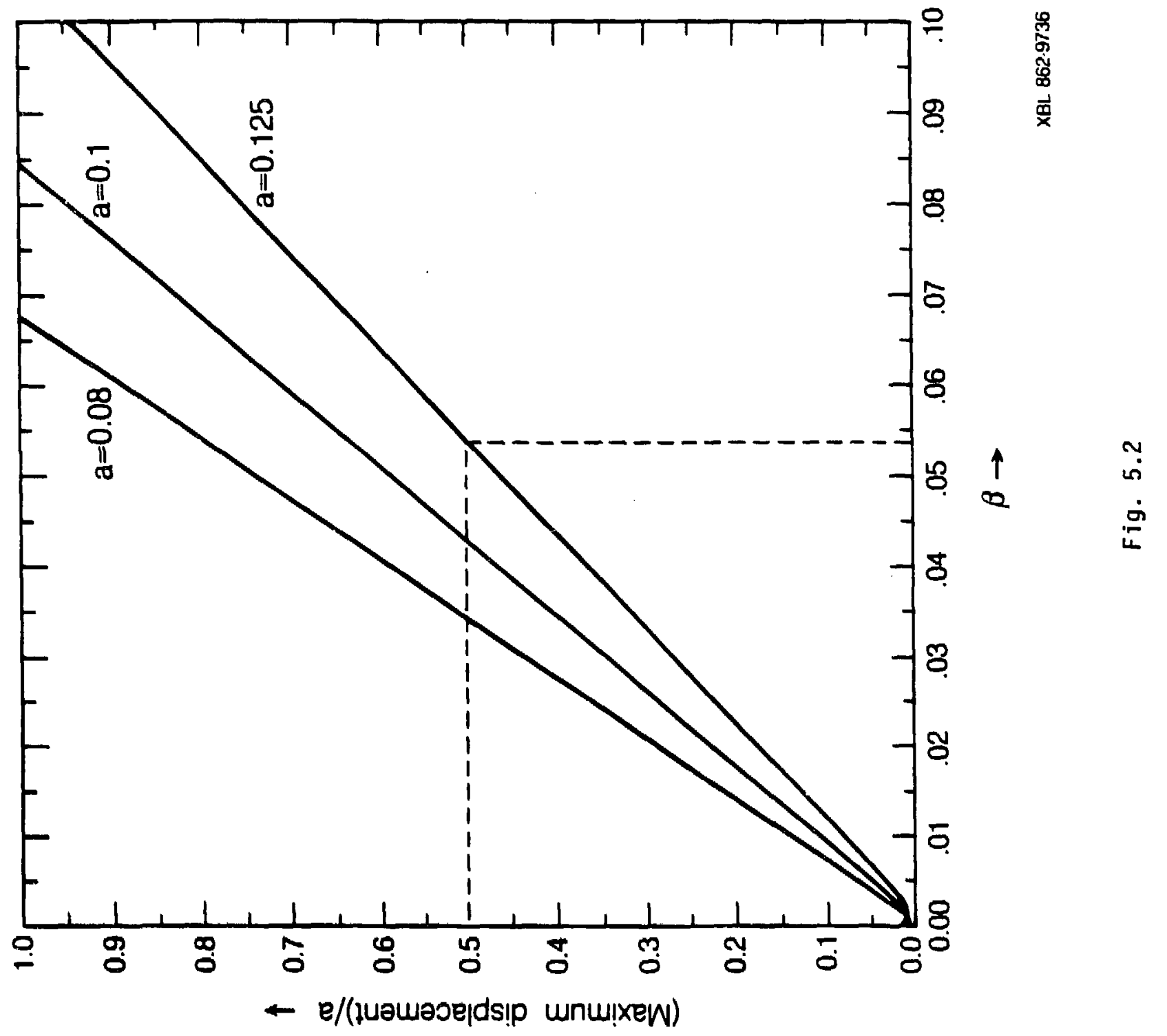




\section{Chapter VI}

\section{Charge Neutralization Current}

In devices, such as ours, which have rectilinear segments connected by curved sections, a current (we shall refer to it as charge neutralization current, although it is referred to by various other names in the literature, such as, charge-separation current or pfirsch-Schluter current) flows through the straight section because of separation of charges in the curved section. This current is mainly longitudinal because of the high mobility of electrons along the magnetic field lines. Unfortunately, this current flowing through the straight section disturbs the axisymetry and causes a Shafranov shift. This shift lowers the equilibrium $\beta$ and thus should be avoided as much as possible. We describe here a scheme of rotating nonctrcular colls to achieve a magnetic field configuration which minimizes this flow of chargeneutralization current.

The condition for vanishing of charge neutralization currents is an equality of $\int d \& / B$ for all field lines on a given magnetic surface [Glagolev et al. (1981)]. Plasma equilibria with zero charge-neutralization currents have also been investigated by $D$. Palumbo(1968), who has shown that in this spectal case the drift surfaces of all particles including trapped particles and magnetic surfaces colncide. The condition for vanishing of charge-neutralization currents reduces to the following requirement in the paraxial approximation:

$$
\int_{-l}^{l}\left[\cosh \frac{n}{2} \cos (\delta-v)-\sinh \frac{n}{2} \cos (\delta+v)\right] k B^{-3 / 2} d s=0
$$

Here $2 l$ is the total length of one curved section, $s$ is the axis arc 
length measured from the middle of the curved section, $B(s)$ is the axial magnetic field strength, and

$$
v(s)=\int_{0} s^{\prime}\left[\left(\delta^{\prime}(s)-x\right) / \cosh n\right] d s \text {; }
$$

$k(s), x(s)$ are the curvature and torsion of the axis; $\exp (n(s))$ is the ratio of magnetic surface cross- section semiaxes, $\delta(s)$ is an angle between the principal normal and the minor semiaxis.

Because of the straight helical nature of our links, the torsion and curvature are constant in the curved section and zero elsewhere. For analytical simplicity if we assume that the ellipticity of the magnetic surfaces is constant along the curved section then, $n(s)=$ constant. (However, in an actual device all the parameters of the curved section's magnetic surfaces would change gradually to that in the straight section, which should be nearly circular. This effect can be taken into account only in a computational analysis.)

Now our scheme of using noncircular colls to achieve a vanishing charge neutralization current is as follows. In the curved section we rotate the noncircular colis in a way that for the resulting magnetic surface, the angle between its minor semtaxis and principal normal to the axts, $\delta(s)$, has the following dependence on $s$,

$$
\delta(s)=a+b s+c s^{2}
$$

Here $a, b$ and $c$ are parameters, which are assumed to be constant in this analysis but could be varied in the actual design.

$$
\text { Then as defined, } \begin{aligned}
v(s) & =\int_{0}^{s}\left[\left(s^{\prime}(s)-x\right) / \cosh n\right] d s \\
& =\frac{b-x}{\cosh n} s+\frac{c}{\cosh n} s^{2} \\
& \equiv b^{\prime} s+c^{\prime} s^{2}
\end{aligned}
$$


50

Then,

where, $b^{\prime} \equiv \frac{b-x}{\cosh n}$ and $c^{\prime} \equiv \frac{c}{\cosh n}$

$$
\begin{aligned}
I= & \cosh \left(\frac{n}{2}\right) \int_{-2}^{2} \cos \left[\left(c^{\prime}-c\right) s^{2}+\left(b^{\prime}-b\right) s-a\right] d s \\
& -\sinh \left(\frac{n}{2}\right) s_{-2}^{2} \cos \left[\left(c^{\prime}+c\right) s^{2}+\left(b^{\prime}+b\right) s+a\right] d s
\end{aligned}
$$

Now using the relation, [Gradshteyn and Ryzhik (1975)]

$\int \cos \left(a x^{2}+2 b x+c\right) d x=\sqrt{\frac{\pi}{2 a}}\left\{\cos \frac{a c-b^{2}}{a} c\left(\frac{a x+b}{a}\right)-\sin \frac{a c-b}{a} s\left(\frac{a x+b^{2}}{a}\right)\right\}$

where $s(x)=\sqrt{\frac{2}{x}} \int \sin x^{2} d x, c(x)=\sqrt{\frac{2}{\pi}} \int \cos x^{2} d x$;

we obtain,

$$
\begin{aligned}
& I=\left\{\sqrt { \frac { r } { 2 ( c ^ { \prime } - c ) } } \left[\cos \left(p_{-}\right)\left(c\left(Q_{-}(l)\right)-c\left(Q_{-}(-l)\right)\right\}\right.\right. \\
& \left.-\sin \left(P_{-}\right)\left[S\left(Q_{-}(q)\right)-S\left(Q_{-}(-q)\right)\right)\right] \cosh n / 2 \\
& -\sqrt{\frac{\pi}{2\left(c+c^{\prime}\right)}}\left[\cos \left(P_{+}\right)\left(c\left(Q_{+}(2)\right)-c\left(Q_{+}(-2)\right)\right]\right. \\
& \left.\left.-\sin \left(P_{+}\right)\left(S\left(Q_{+}(2)\right)-S\left(Q_{+}(-2)\right)\right)\right] \sinh n / 2\right\} \text {; }
\end{aligned}
$$

$$
\text { where } \begin{aligned}
p_{ \pm} & =\frac{\left(c^{\prime} \pm c\right)( \pm a)-\left(b^{\prime} \pm b\right)^{2} / 4}{\left(c^{\prime} \pm c\right)} . \\
q_{ \pm}(s) & =\frac{\left(c^{\prime} \pm c\right) s+\left(b^{\prime} \pm b\right) / 2}{\sqrt{c^{\prime} \pm c}} ;
\end{aligned}
$$

Defining, $C_{ \pm} \equiv\left(C\left(Q_{ \pm}(\ell)\right)-C\left(Q_{ \pm}(-2)\right)\right\}$

$$
\begin{aligned}
& S_{ \pm} \equiv\left\{S\left(Q_{ \pm}(2)\right)-S\left(Q_{ \pm}(-2)\right)\right\} \\
& R_{ \pm}^{2} \equiv c_{ \pm}^{2}+S_{ \pm}^{2}
\end{aligned}
$$

$$
\tan \theta_{ \pm} \equiv S_{ \pm} / C_{ \pm}
$$




$$
A_{ \pm} \equiv \sqrt{\frac{\pi}{2\left(c^{\prime} \pm c\right)}}
$$

we get,

$I=\left[A_{-} R_{-} \cos \left(P_{-}+\theta_{-}\right) \cosh \frac{n}{2}-A_{+} R_{+} \cos \left(P_{+}+\theta_{+}\right) \sinh \frac{n}{2}\right]$

A careful analysis shows that only $P_{ \pm}$depend upon the parameter 'a'. Since I is expressed as the difference of two cosine terms, it also behaves like cosine ttself. Thus for a given value of $b$ and $c$, we should be able to choose parameter ' $a$ ' so that the integral vanishes. In this way we should be able to achieve a significant reduction of the chargeneutralization currents. Of course, a numerical study is needed to ascertain this. Unfortunately, this rotation may lead to an enhanced loss of particles from the bend sections. In this thesis, we have not studied this scheme numerically. 
Chapter VII

Numerical Analys is and Results

Vacuum Magnetic Field Properties

We studied two aspects of THELMA numerically. One was the study of vacuum magnetic fleld properties and another was the single particle orbits. For the study of vacuum magnetic field properties, we used a modifted version of the 'HELIAC' [Ehrhardt (1985)] computer code. This code calculates analytically the magnetic field at any point and can estimate the magnetic axts for a given configuration. It can also follow any field line and make puncture plots, in addition to calculating various properties of magnetic surfaces. The code was originally written for the purpose of studying the Hellac type device, which was assumed to have a circular axis lying in a plane. With this assumption, the plane on which puncture plots were specified was only an azimuthal plane. However for THELMA this assumption is not valid. Thus we had to take into account the 3-dimensfonal nature of the geometrical axts of the device and modify the code suttably. In particular the following modiftcations were done:

(1) The algorithm, locating the magnetic axts, was modifled by adding the posstbitity of having the z-coordinate nonzero. (Here we are making the impitcit assumption that the mid-points of all THELMA sections lie in the $x-y$ plane, see Append $(x-c)$.

(2) The magnetic field calculation subroutines were replaced by a new set of subroutines taken from the COIL3 program [Karney (1985)], which uses dynamic dimensioning and thus the number of colls was not 
limited at the time of complling. The subroutines are also Vectorized for faster calculation of magnetic fields.

(3) Buffered input for magnetic field coll elements was added to ensure accuracy of data to very high order.

(4) Furthermore, we added the option to specify the plane on which a puncture plot is created, by giving the direction cosines and three points on the plane. A related modification was made in subroutines which calculated flux and area assoclated with a set of puncture points created by a field line, lying on a closed curve. Also, the calculation of magnetic well depth was modifled taking into account the 3-dimensional axis.

Here we show some vacuum magnetic field properties of THELMA with circular colls using the above-mentioned code. The spacing of the coll was chosen such that the magnetic field ripple was less than $1 x$ on the magnetic axis (as shown in Fig. 7.1, with no mirrors in the straight section). The three-fold symmetry, as is to be expected from the design of the device, is reflected in the variation of the magnetic field on the magnetic axis with azimuthal angle. Sitght variations are due to the Inaccuracy in locating the magnetic axis. The zero of the aztmuthal angle represents the middle of a helfcal section. Figure 7.2 shows the variation of the magnetic field magnitude $B$ on the magnetic axis, when a mirror fleig is introduced in the straight section by changing the current in the cotls.

Fig. 7.3 and 7.4 show the puncture plot of the field lines in the middle of the stralght and helica: sections respectively. As is clear from the figures, the field lines seem to form well-defined nested 
Magnitude of $\mathrm{B}$ along the magnetic axis

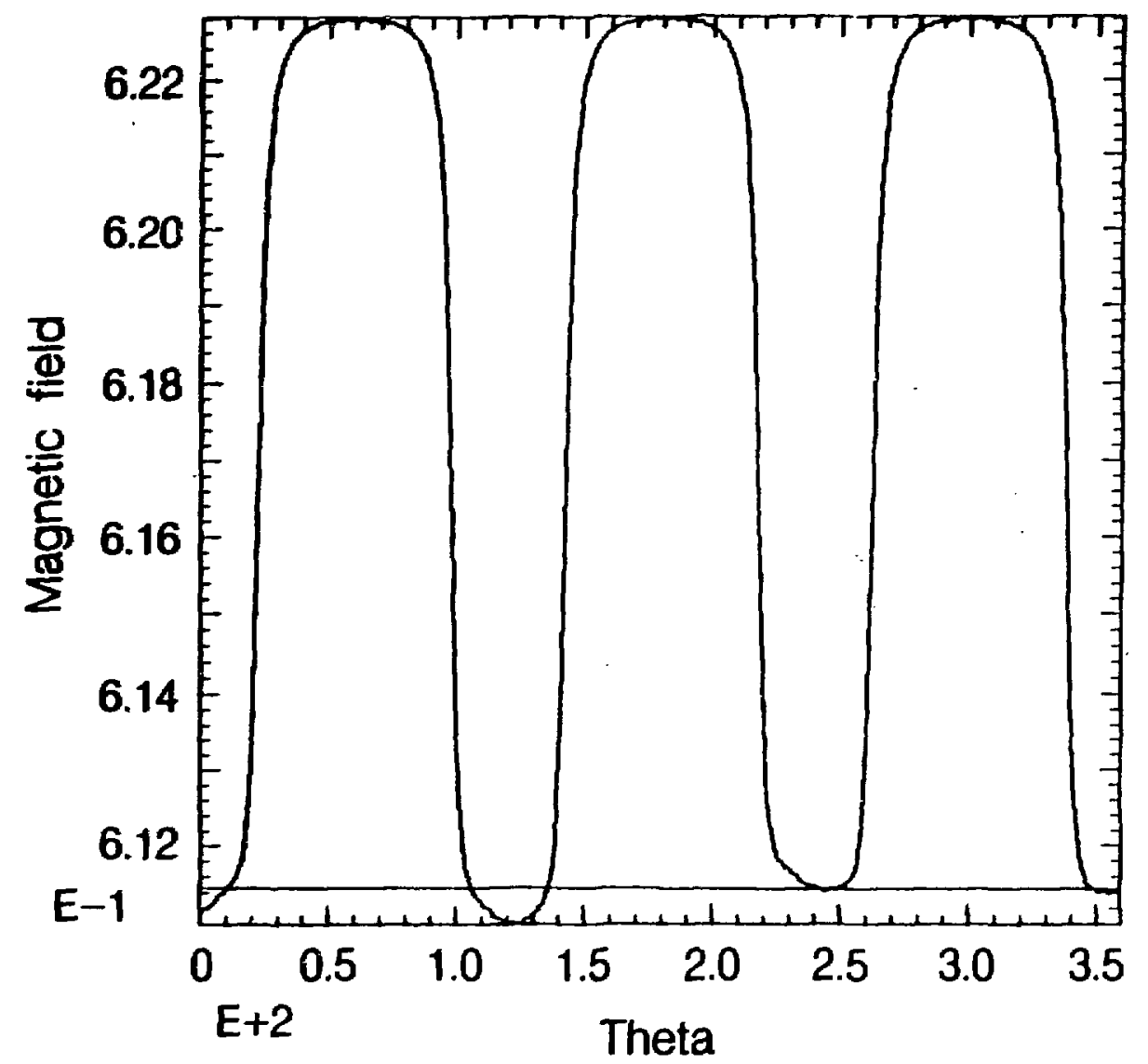

XBL B62-9737

Fig. 7.1 
Magnitude of $B$ along the magnetic axis

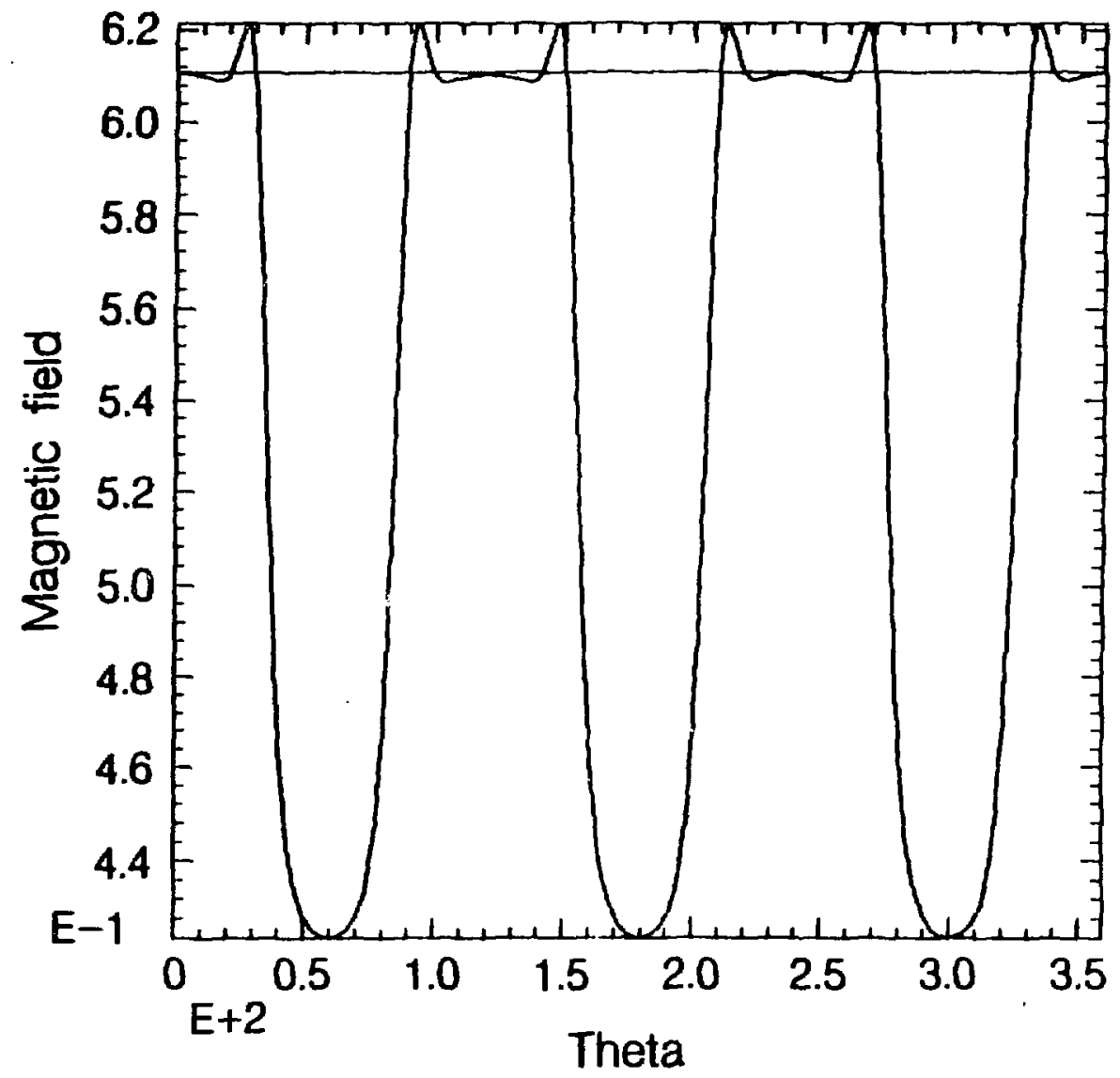

XBL 862-9738

Fig. 7.2 


\section{Puncture Plot: Mid-Helix}

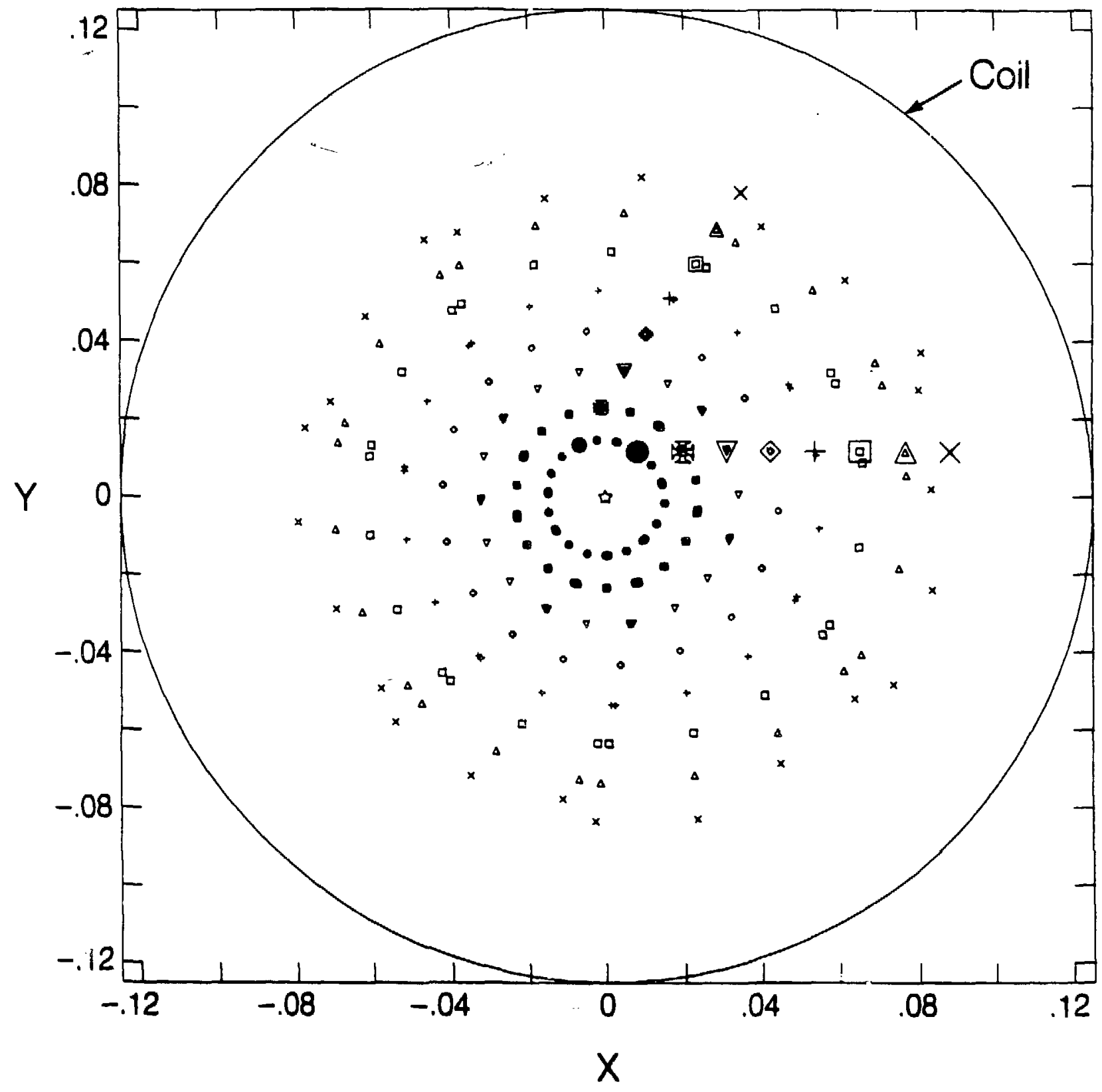

XBL 862.9745

Fig. 7.3 
Puncture Plot: Mid-Straight

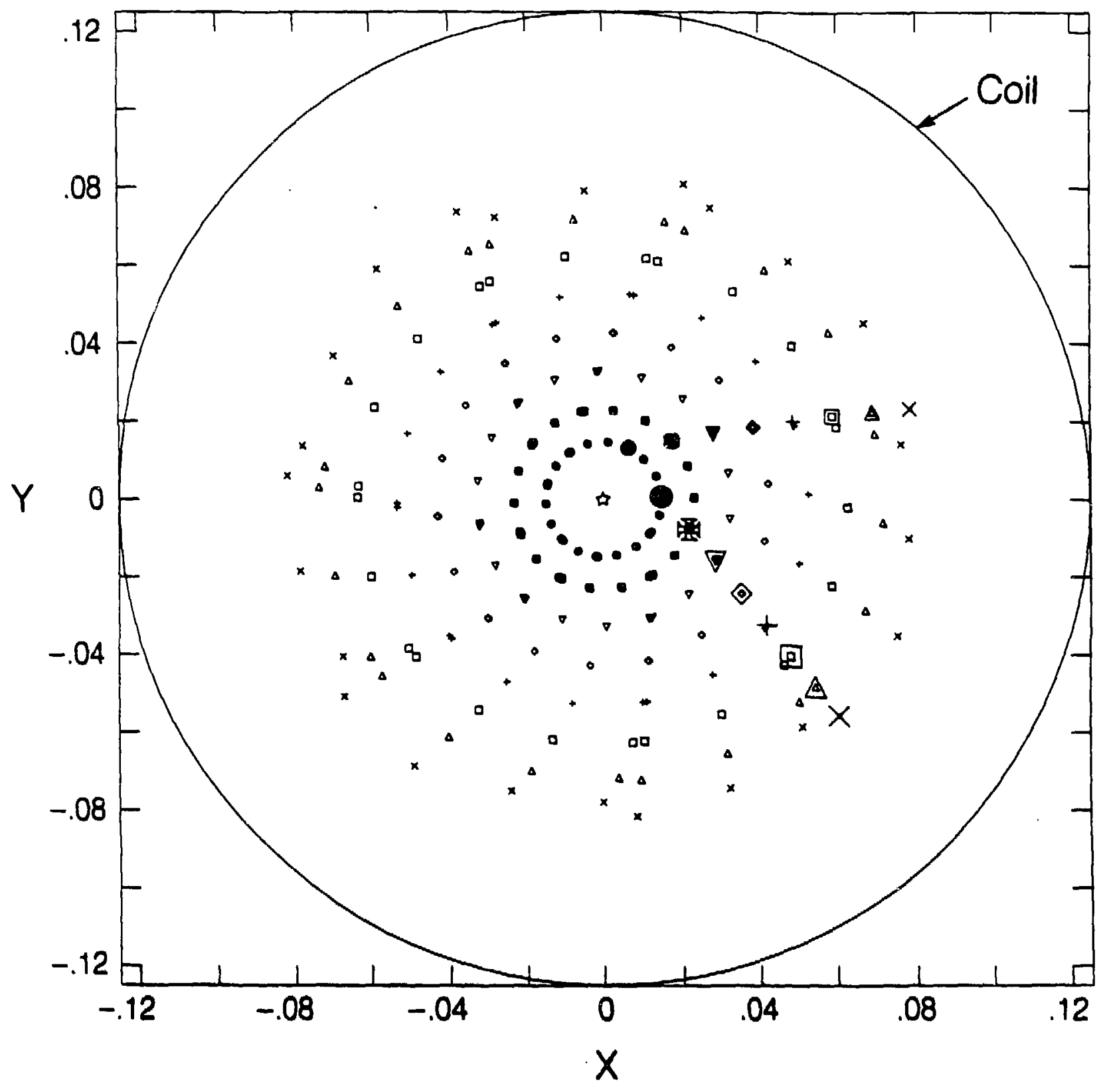

XBL $862 \cdot 9747$

Fig. 7.4 
magnetic surfaces. Different symbols represent different starting points for the field lines. If one looks carefully, the outermost magnetic surfaces start showing some osctllatory behavior and break apart as we go further away from the magnetic axis. The radial distance at which this occurs is sensitfve to the strength of the ripples in the magnetic fleld. Less ripple in the magnetic fleld configuration increases the area in which fleld lines form closed magnettc surfaces. This has a direct effect on the behavior of single particle motion, as expected theoretically.

The magnitude of the rotational transform is seen to first increase and then decrease with increasing distance from the magnetic axis as shown in Fig. 7.5, however the net variation in the rotational transform is very small. Thus shear is also small. Agreement with the approximate calculation of the rotational transform on the axis is good. Also one should note that the rotational transform is not passing through any region of low-number rational multiple of $2 \pi$.

\section{Single Particle Motion}

After highlighting the important aspects of the vacuum magnetic field properties, we now give the results of the computational study of single particle drift orbits. For this part of the study the computer code "TIBRO-X" [Foote (1976)] was used. However the following changes were incorporated:

(1) The magnetic field calculation was completely vectorlzed.

(2) The output was put into a totally different form, which was more sultable for understanding the complex 3-dimensional geometry of THELMA. The particle guiding center position was determined with 


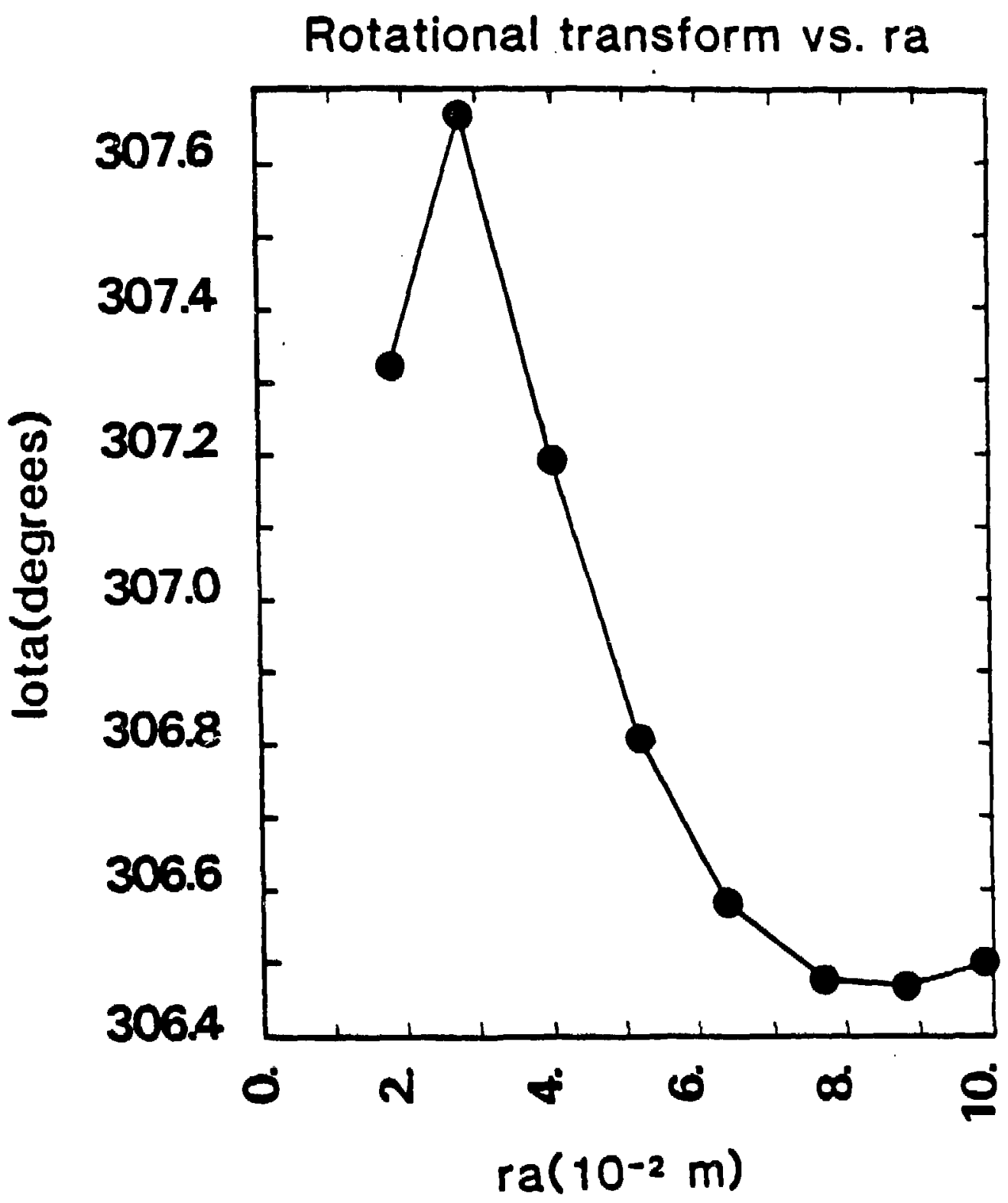

XBL 862.9739

Fig. 7.5 
respect to the geometric axis and the length along the geometric axis from some reference point (i.e., a coordinate system similar to the Mercier Coordinate system was used). Provision was made so that the reflected particles could be shown as such.

(3) An option was added whereby puncture plots of drift orblts could be shown in the middle of the hellcal and stralght sections.

(4) A post-processor was developed so that data from various runs could be combined in a meaningful form.

To compare with our approximate theoretical model we show here in Fig. 7.6, a typical output for the case when there are no mirrors in the stralght sections $(R=1)$, and in which:

radius of cot1, $a=0.125 \mathrm{~m}$

total axis length $=9.6 \mathrm{~m}$

Curvature, $k=2.2 \mathrm{~m}^{-1}$

Torston, $x=1.1 \mathrm{~m}^{-1}$

Particle Energy, $E=0.2 \mathrm{KeV}$

Magnetic Fleld $=0.3 \mathrm{~T}$ (In the middle of straight section) Velocity ratio $V_{\|} / V_{\perp}=2.0$

Fig. 7.6 shows the variation of the radial distance of the guiding center from the geometric axis with respect to the distance along the axts. The radial distance remains constant in the straight section but changes in the helical section. Puncture plots in the middle of the helical section show that the drift orbits lie on closed curves, hence the orbits are well confined. The center of the orbits is displaced with respect to the geometrical center by about $1.0 \mathrm{~cm}$, which compares well with theoretical value of $1.3 \mathrm{~cm}$. Fig. 7.7 shows the variation of drift 


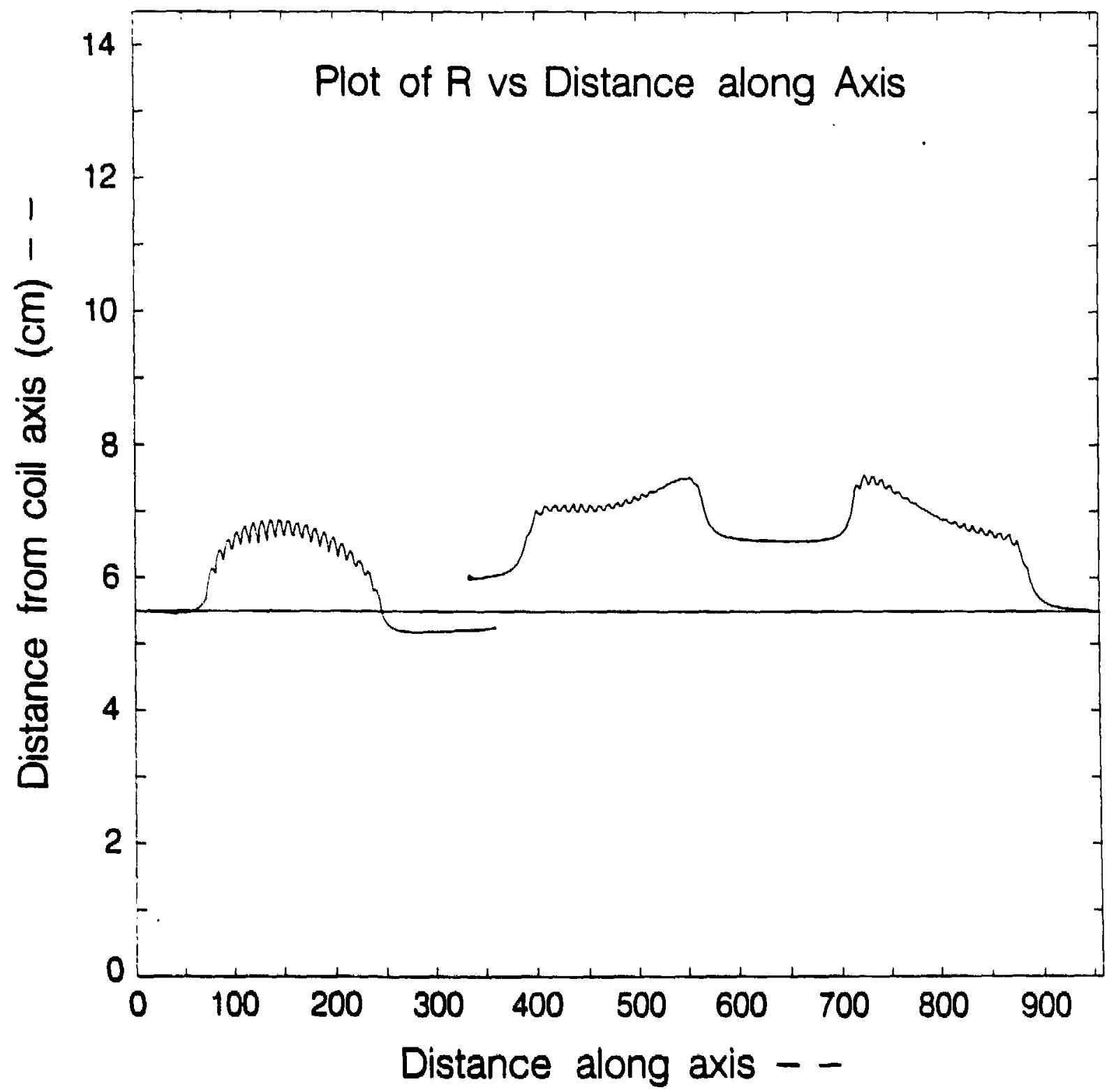

XBL 862.9740

Fig. 7.6 


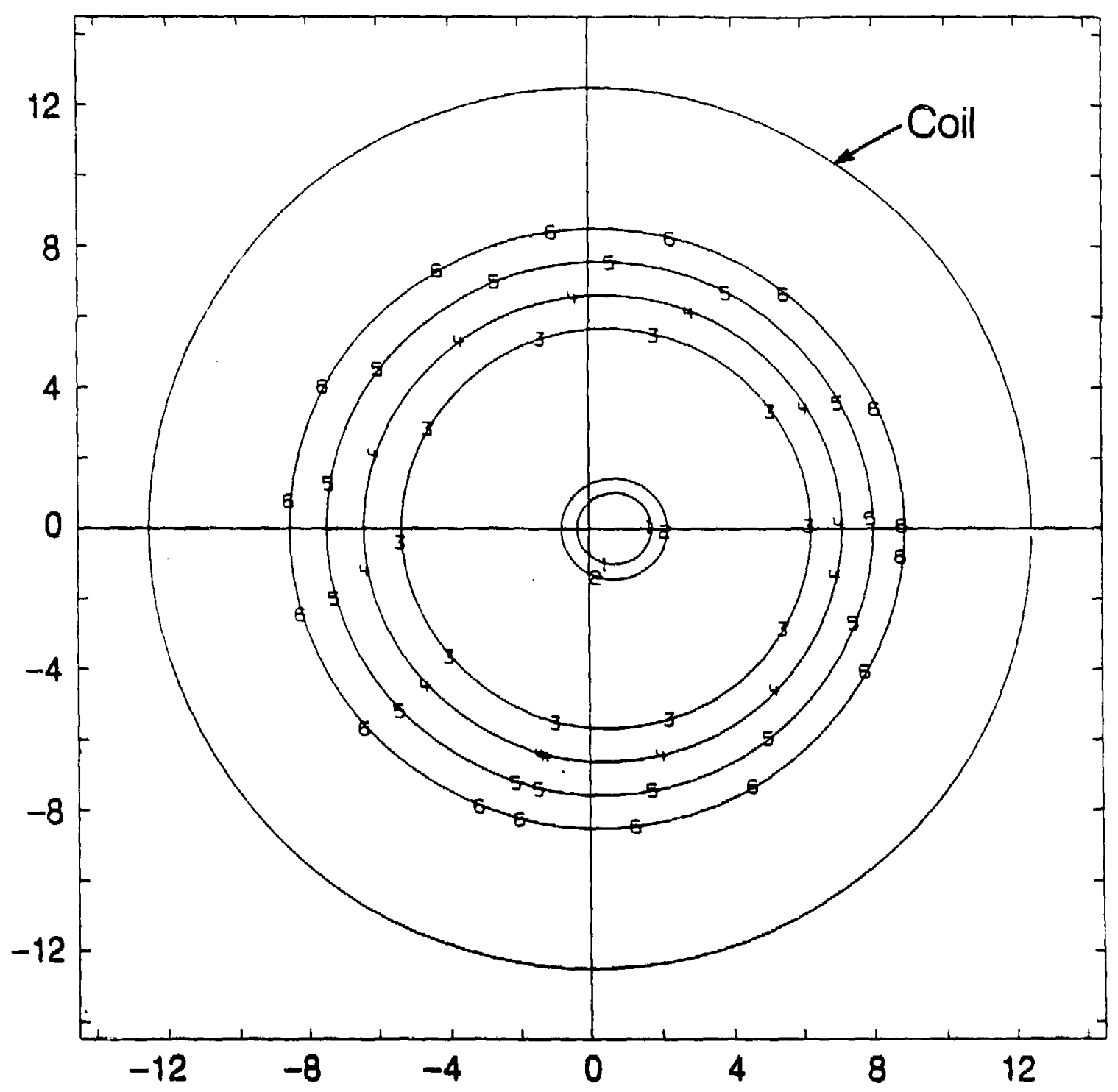

XBL 862-9741

Fig. 7.7 


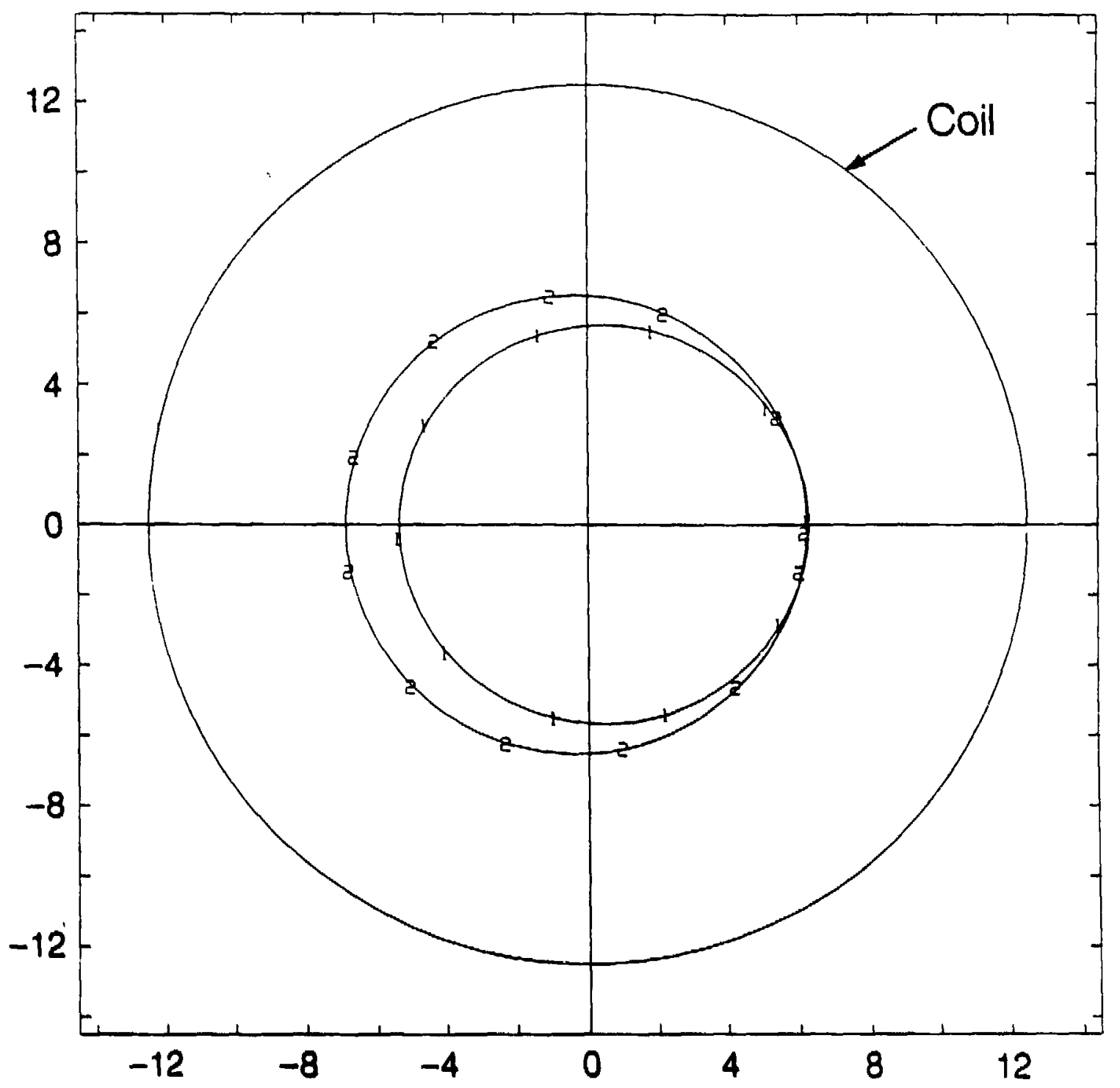

XBL $862-9742$

Fig. 7.8 


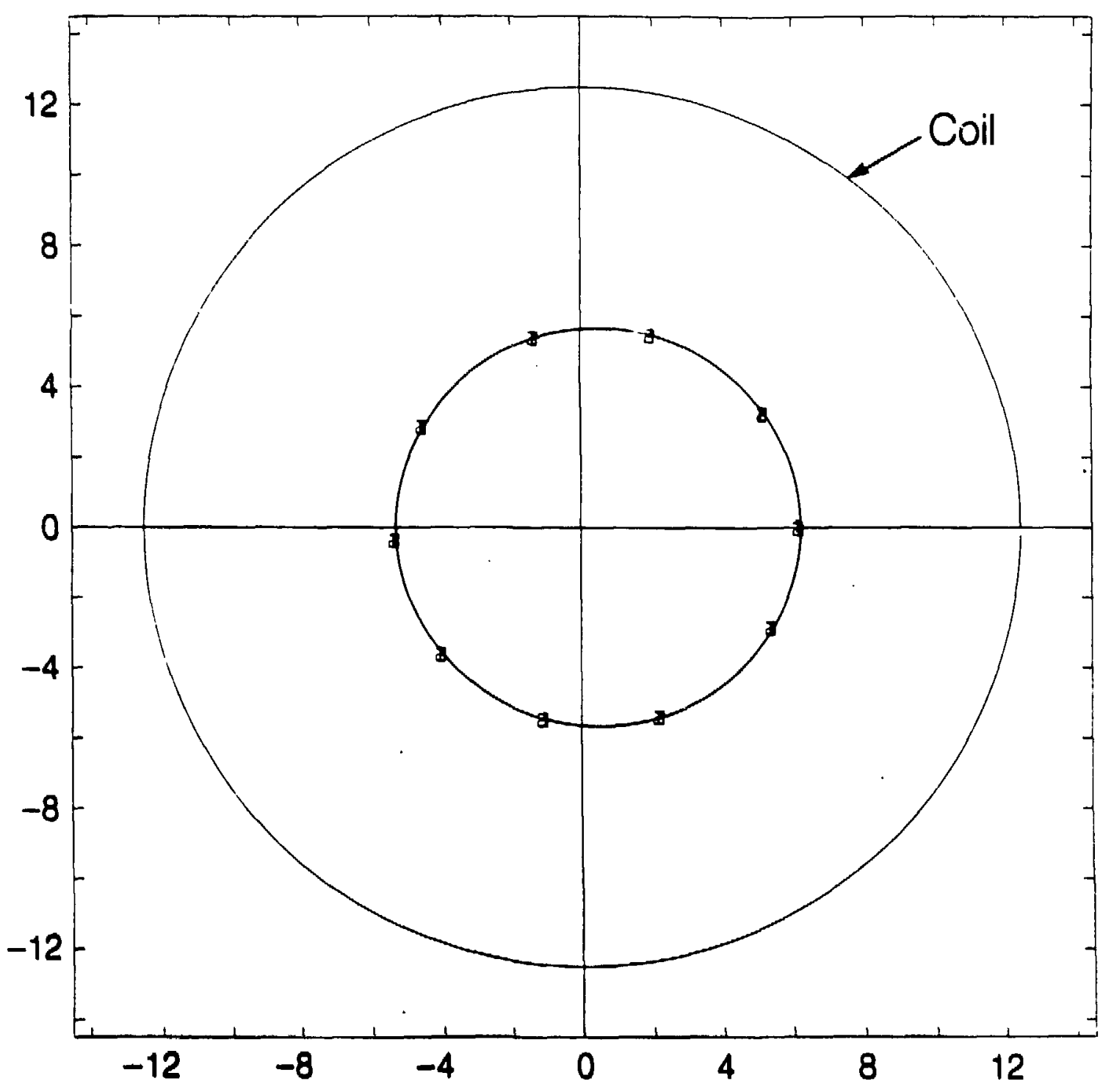

XBL 862.9743

Fig. 7.9 
orbit Puncture plots with radial distance in the middle of a helical section. The drift orbits do not remain closed beyond a certain maximum radial distance. The regions of closed orbits and open orbits overlap a little bit at the boundary. Whether an orbit is closed or open in this transition region depends on the pitch angle. Figs. 7.8 and 7.9 show the dependence of drift orbits on sign of charge and pitch angle. We find that orbits are displaced for different sign of charge. We do not see much change in orbit with pitch angle as we have shown in Fig. 7.9 for the orbits of particles having velocity ratios of 2,5 and 50 . These are in good qualitative agreement with theoretical expectation.

Noncircular Cofls

After studying the system with circular colls in the helical section we decided to investigate whether incorporating noncircular colls would lead to the improvements we expect theoretically, namely, providing stabilization of plasma by creating an average magnetic well and producing vanishing charge neutralization current in the straight section. However. we have only been able to investigate one part of this problem 1.e. Whether it creates an average magnetic well or not. For this purpose we used a system of colls in which the coll in the center of the helical section was bean-shaped. However, the parameters of the colls were gradually changed so that the shape of the coll changed gradually from bean-shaped to circular near the transition from helical to stralght section. The parameters to represent the colls are Monticello Bean Parameters, which have been discussed in Appendix-C. Internally the colls are represented by 25 linear segments. The linear 
segments are chosen such that the area enclosed by them is equal to the area enclosed by the actual curve. This ensures a more accurate approximation to the magnetic field.

The results of this partial study of noncircular colls in the helical sections are as follows. We must however note that no optimization of parameters has been attempted at this stage. Thus some results may be Improved upon compared to those obtained here. The puncture plot of the magnetic field lines for the middle of the helical and the straight sections are shown in Fig. 7.10 and Fig. 7.11. As one notices, the magnetic fleld lines seem to form closed curves on the planes. Also, the magnetic surface cross-sections in the mid-helical plane form oval or D-shaped curves, but they remain circular in the middle ôf the straight section. This result is important, as it showis that it is possible to create a noncircular magnetic surface in the curved section and at the same time have circular cross-sections in the midole of the straight section. Thus the axisymmetry of the straight section is preserved to a good extent. We also notice that the fleld lines remain confined in the central portion of the bean-shaped coll. However the field lines lead out of the device in the lobes of the bean, these are not shown in the figure. Thus the net effective volume in which plasma can be confined decreases. We also show the effect on the rotational transform in Fig. 7.12. The rotational transfrom decreases as expected, but not drastically. This means that single particles in the region in which field lines are confined, should also be confined as long as the parallel to perpendicular velocity ratio is high enough (as discussed earlier in Chapter IV). However we have not tested this aspect computationally. 
Puncture Plot: Mid-Helix

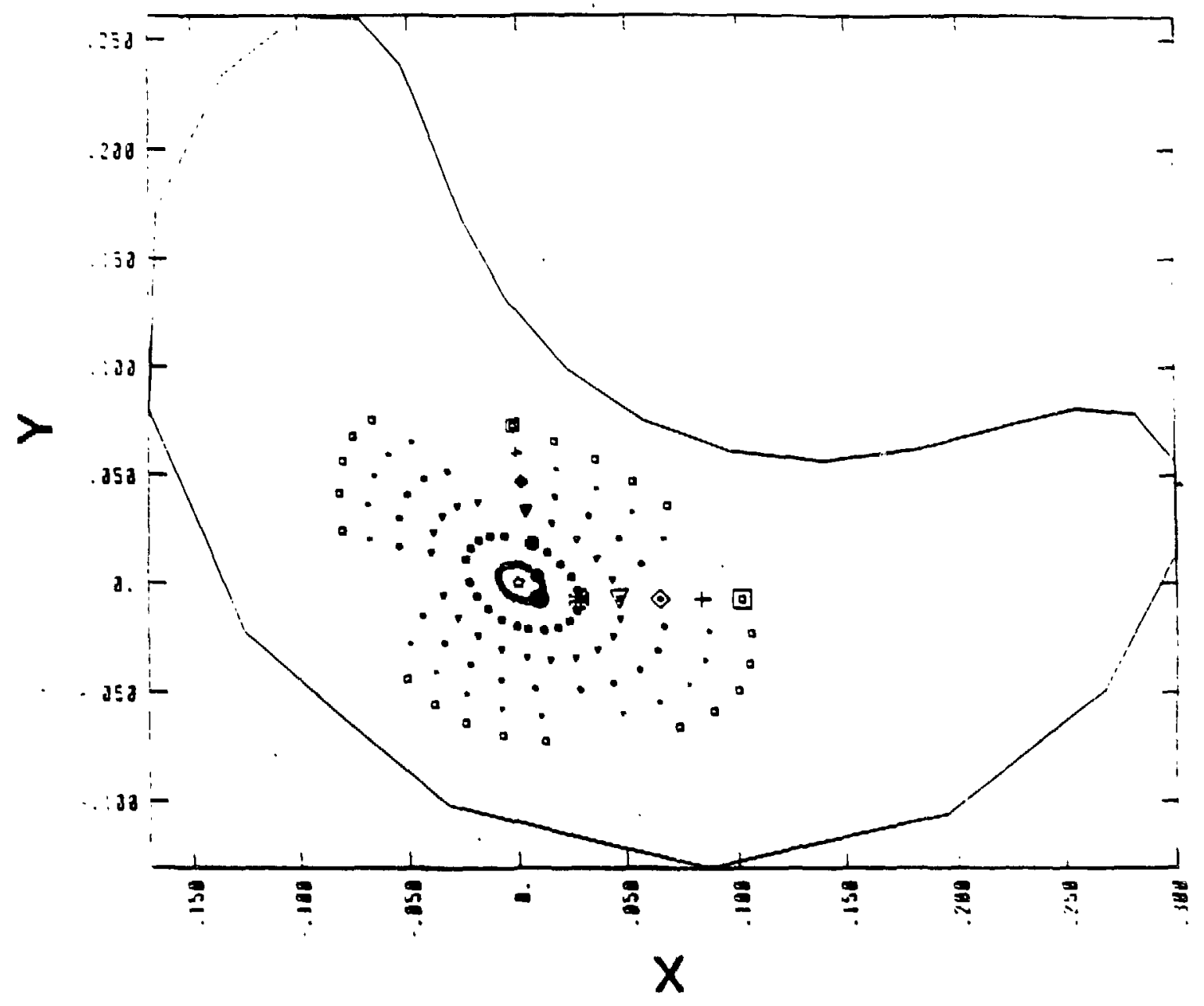

XBL 8610-3713

Fig. 7.10 
Puncture Plot: Mid-Straight

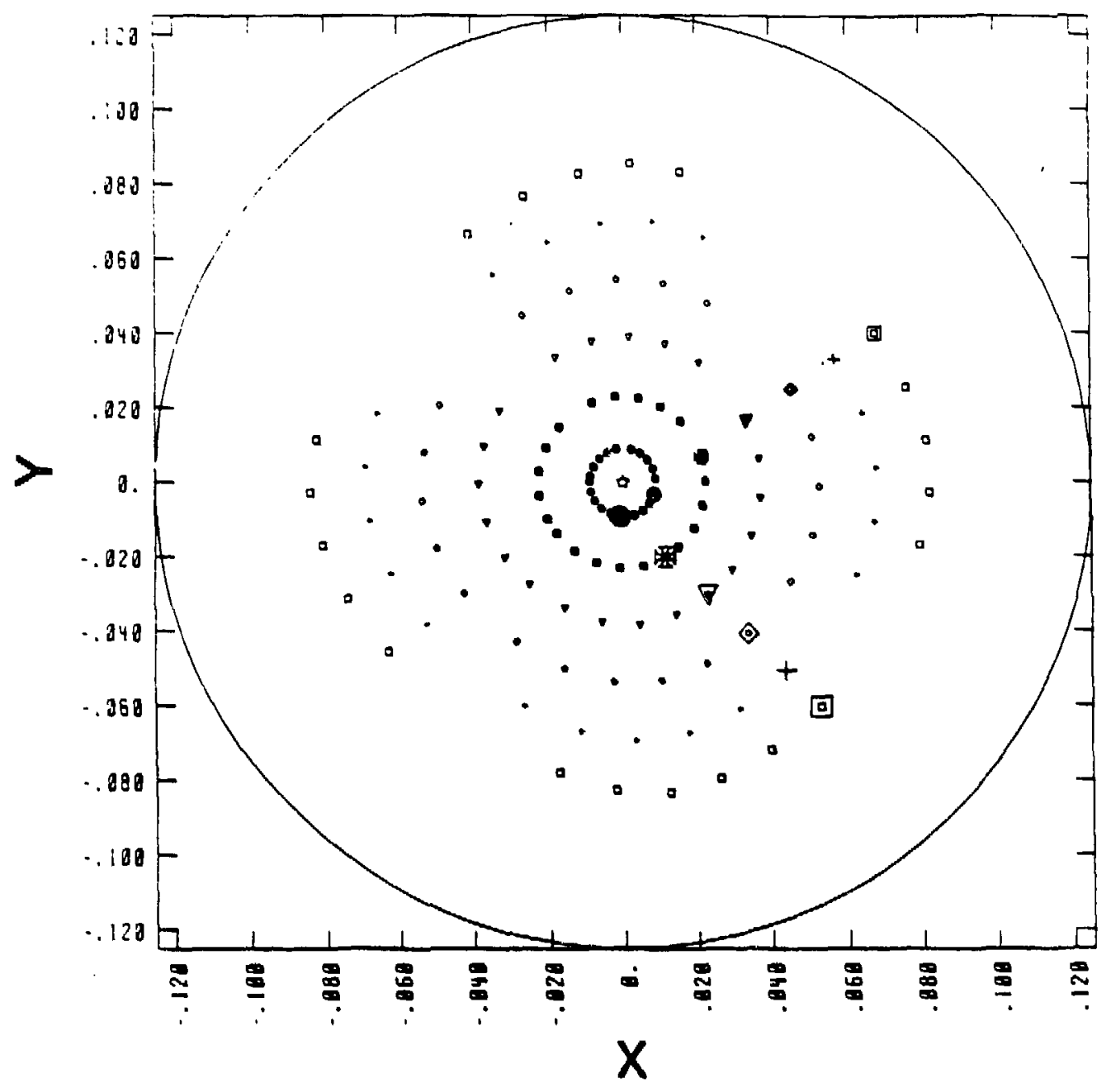

XBL $8610-3714$

Fig. 7.11 


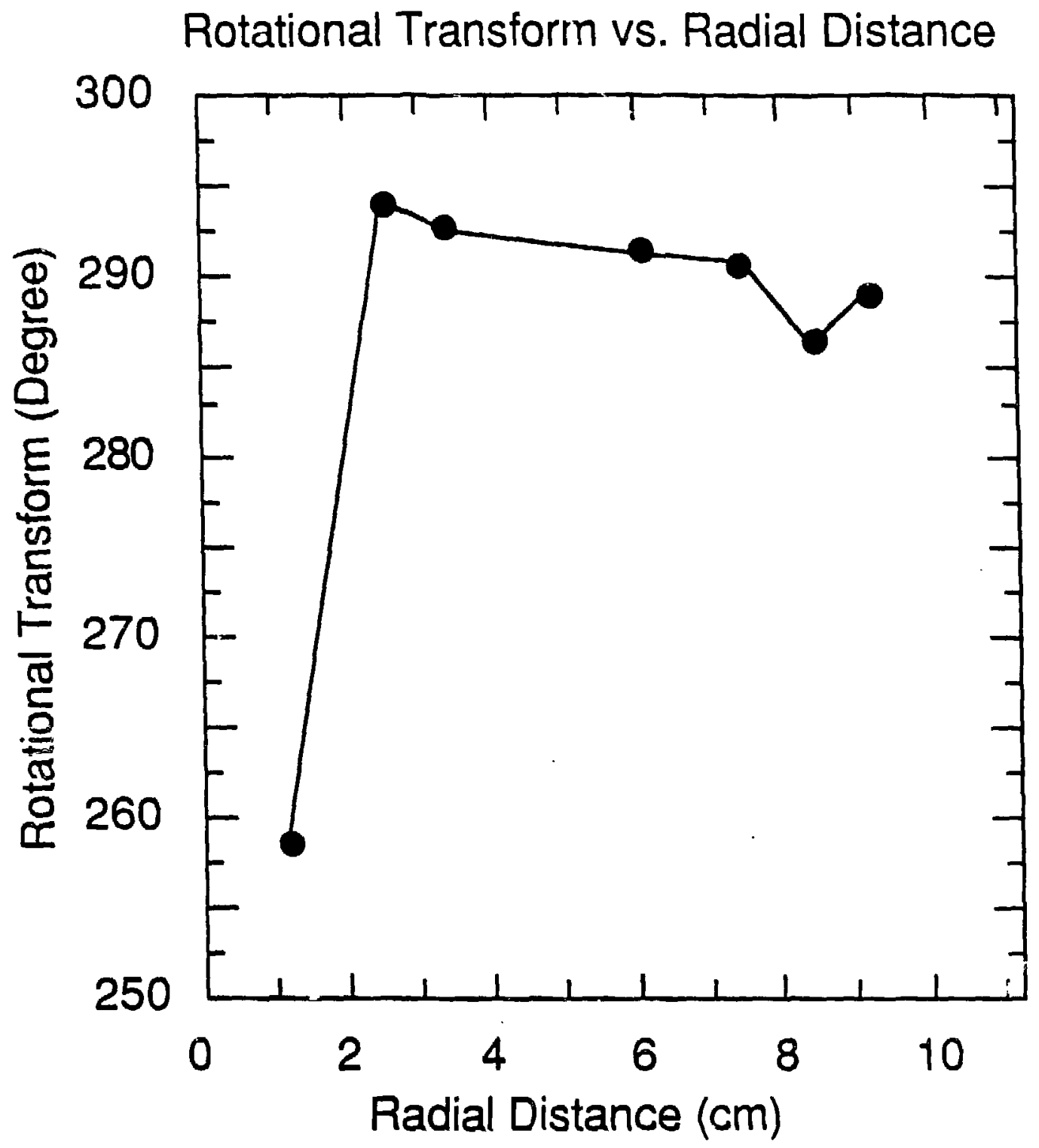

XBL $8610-3715$

Fig. 7.12 
The variation of the rotational transform with transverse distance shows that the amount of shear increases by a large amount, which results in stabilization of certain modes of plasma instability. However, the numbers calculated for the stability beta limit (shown in Fig. 7.13) takes into account only the rotational transform and its variation. There may be other aspects, such as ballooning modes or transverse shift of the plasma column, which would limit the stability beta to lower values. However, the most important aspect is the creation of an average magnetic well. We show in Fig. 7.14 the variation of magnetic well depth with transyerse distance. As we notice the magnetic well depth is positive for lower values of radial distance but negative for higher values. This means that the noncircular colls we used in our investigation were not sufficient by themselves to create an overall average magnetic well. Elther a change in the parameters of the colls or use of a different method (some of which we suggest at the end). may be able to do this. 


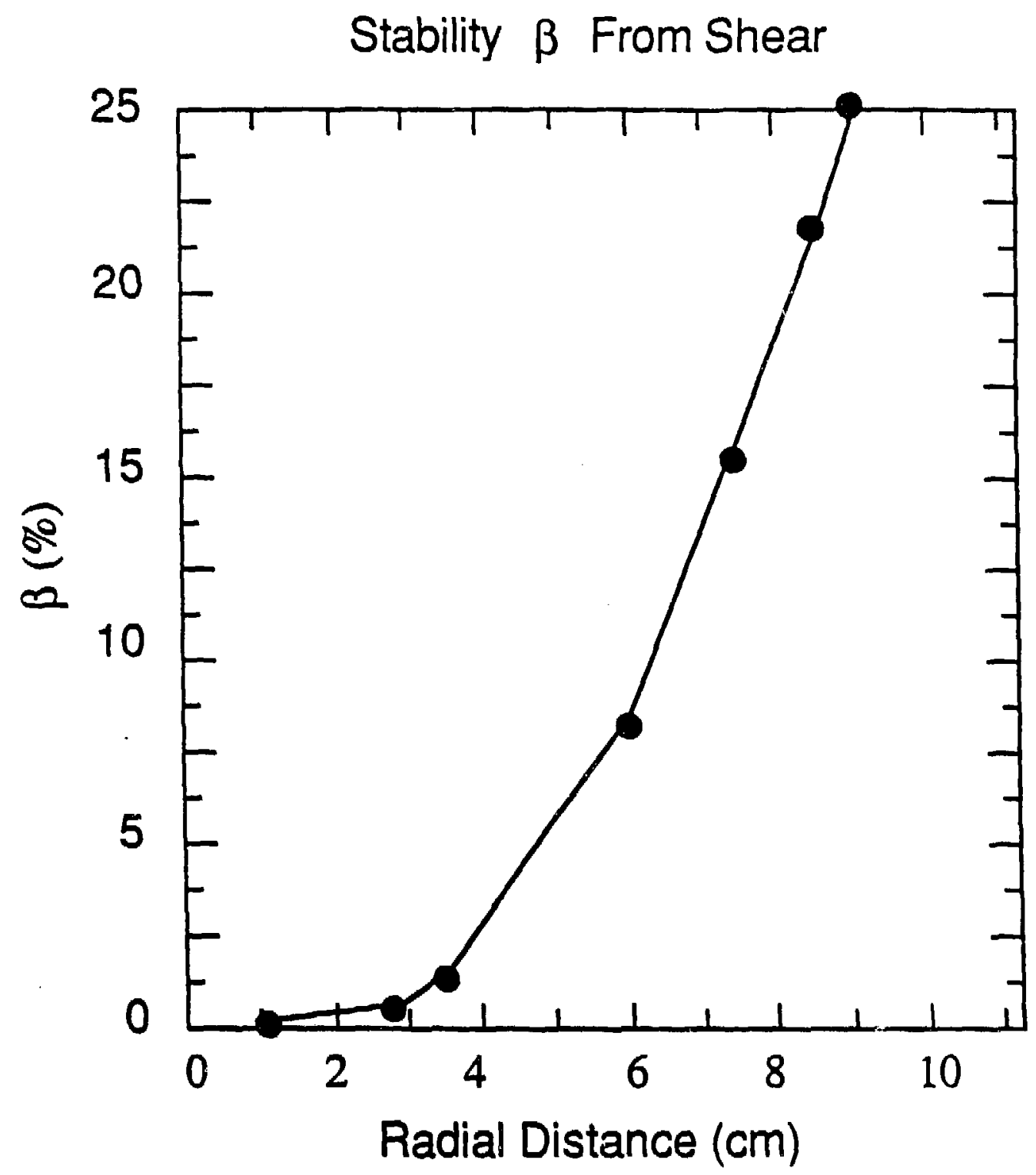

XBL $8610-3716$

fig. 7.13 


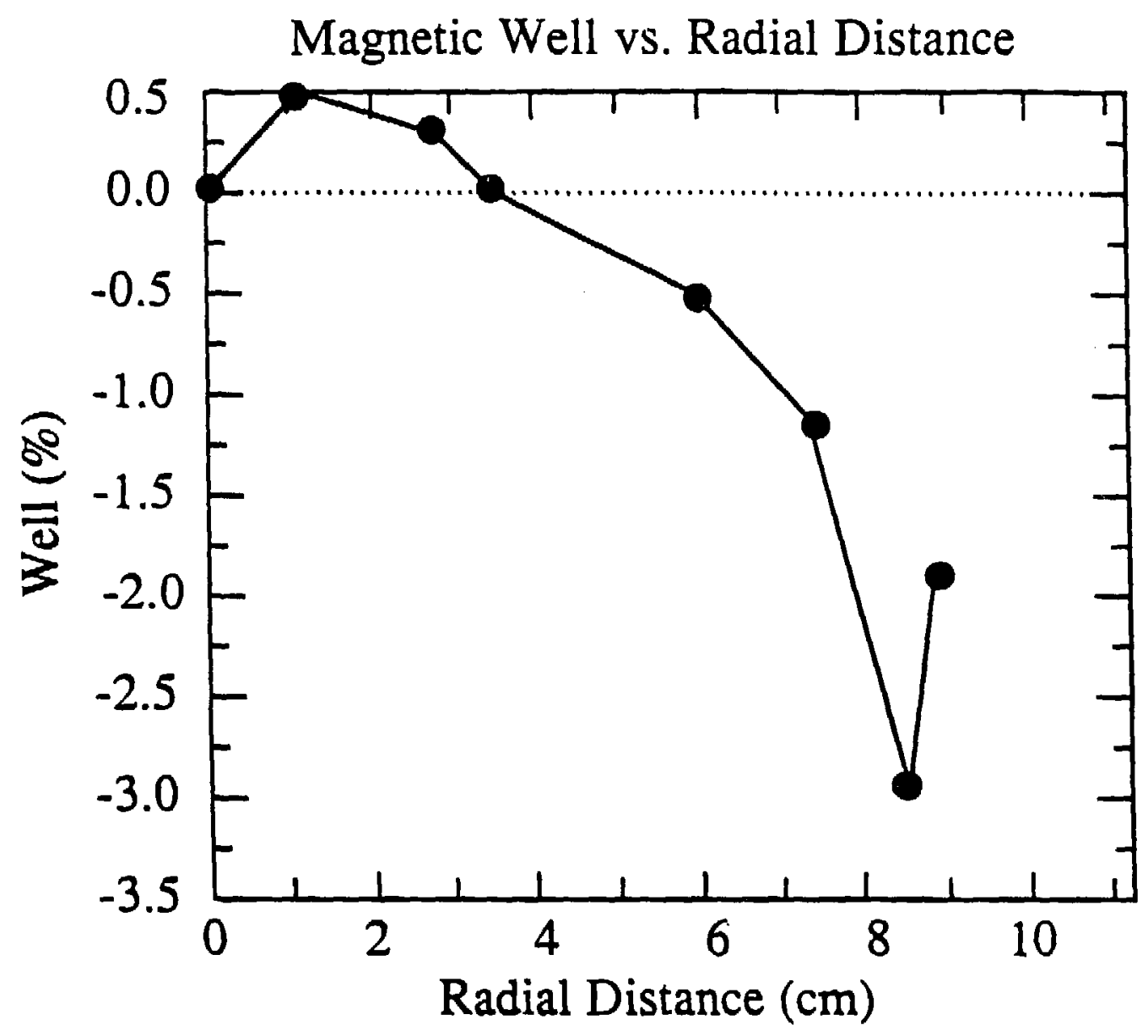

XBL 8610-3717

Fig. 7.14 
Chapter VIII

\section{Stablilty of Linked Mirrors}

For any magnetic confinement scheme to work successfully MHO stability of the device needs to be ascertained. In any mirror device a source of flute instablifty arises from the curvature of the field lines near the mirror throats. In our linked mirror scheme one more source of such instability is the curvature of the field lines in the links themselves. Of course the question of stability arises only when the confinement device has an equilibrium state. Many devices have poor containement of plasma because they do not have an equilibrium state [Grad (1967)]. One level at which we can examine confinement is the study of individual particle orbits in a given steady field. Containment of individual orbits is necessary but not suffictent for containment of plasma. In the case of THELMA we made a large number of individual runs following orbits of particles, the results of which were summarized earlter in Chapter VII. We also followed the field lines and we can assert with a reasonable degree of conftdence that well defined nested magnetic surfaces are formed in the device, which also exist in the case where noncircular magnette surfaces are created in the links. The existence of nested magnetic surfaces with high rotational transform Impltes extstence of plasma equilibrium. Thus in the next stage, we need to concern ourselves with the stablitty of the equilibrium with respect to various perturbations that may arise.

One type of magnetic field arrangement, which is stable to all MHO perturbations is the Min-B configuration in which the magnetic field 
strength increases outward at all points. In the case of open fieldline systems many examples have been designed and studied extensively. However, according to a theorem proved by Jukes (1964) it is not possible for an absolute minimum of vacuum magnetic field strength to exist in any system consisting of closed flux surfaces without having internal conductors. More accurately, the theorem states: "Given a force-free, or vacuum field in which fleld lines envelope a closed flux surface $F$ enclosing finite volume. Then there must exist some region on $F$ where the fleld strength increases inwards across F."

Thus the toroldal devices, without internal conductors, cannot achleve absolute min-B stability. Thus the attempt in most of the toroidal devices is to generate an average positive magnetic well configuration, which is an integral property of the device. From the practical standpoint, this may be sufficient for the purpose of fusion reactors. As mentioned earlier, a toroldal device is defined to have an average magnetic well if $V^{\prime \prime}(\$)<0$; where $V$ is the volume enclosed by a magnetic surface and is the longitudinal magnetic flux enclosed by 1t. A good summary of various theoretical considerations that are required for designing a magnetic confinement device, where the magnetic fleid is generated by currents in external colls, is given by Shafranov (1983).

We have not yet studied the stability aspects of THELMA in any detall. However, we would like to mention some thoughts based on the studies done for similar devices. Some ways of providing stability to the Dracon Confinement device was presented by Arsenin et a 1. (1983). They have proposed three methods of stabilizing flute perturbations: 
a) by creating magnetic wells in the curved linkings using auxiliary helical windings;

b) by means of an anisotropic high- $\beta$ plasma confined in an additional minimum-B trap, a so-called "anchor";

c) by relativistic electron rings (bumpy-torus-type stabilization).

The creation of magnetic wells in tire curved linkings can be done by producing noncircular " 0 " or "bean-shaped" surfaces. This can be achleved by using efther a set of helfcal colls or using discrete modular coils. With the helical linkings having only ctrcular magnetic surfaces, the B limited by stability is negligible and thus such a device does not have any practical use. It is known from calculations for a helical system that for constant curvature and torsion stability can be achieved for $B=15-20 \%$ [Shafranov (1968)] so that even in the presence of unfavorable sections which are unavoldable with variable curvature and torsion, stability can be expected for moderate $B$. However, we have to a) ways incorporate some form of circularizer to convert noncircular magnetic surfaces to circular magnetic surfaces. In the simple device we studted with nonctrcular colls we did this by changing the parameters of the noncircular colls in a way that the bean shaped colls changed to circular ones in the straight section (as shown in Fig. C.3). However, this method is not very effictent for creating magnetic wells as this transition takes an axis length of a few coll radil. Thus, as mentioned by Arsenin et al. (1983), one may use a circularizer conststing of $m=1$, 2. 3 multipole windings with corresponding currents, which makes this conversion over a few plasma radil.

We also note here that if the lengths of the straight section, transition section and curved section are properly chosen then the device 
may be stable to the flute instability all by itself, by virtue of its rotational transform, 1.e., without anything else done to create a magnetic well. As shown also by Arsenin et al. (1983), when the magnetic fleld in the straight section is weaker than that in the CREL, the transition section with convex field lines has a destabilizing effect. For low $B$ the contribution made by this region to the potential energy of a flute perturbation is proportional to $B L / L_{m}^{2}$, where $L$ is the plasma scale-length, and $L_{m}$ the characteristic length of the varlation of the magnetic fleld in the mirror. For a mirror ratio of $\sim 2, L_{m}$ is of the order of the mirror length, $L_{t^{-}}$. Thus the system is stable if the mirror is suffictently long. Physically, this means that the growth rate of the flute instability has a longer time-scale than the time taken for an Alven wave to propagate from one mirror section to the next one. Finfte larmor radius effects typically tend to stablitze most of the higher order instability.

Stabilization by using min-B mirror or quadrupole coll is essentially an attempt to link "anchored" tandem mirrors. However it is mentioned that the parameters requitred for the quadrupole coll is much less stringent than a stand-alone Tandem mirror. Some preliminary Fokker-planck calculations are mentioned, that show that the sustainenance of a hot-ion component using neutral infection requires an injection energy of $-300 \mathrm{KeV}$, and that the required energy consumption should not exceed 10x of the thermonuclear power released in the reactor. In addition, no thermal barrlers are required for this purpose. On the other hand, stabilization using quadrupole colls means that the device as a whole becomes very complex and might not be attractive enough. 
Another method suggested is the use of hot-electron rings to create an average magnetic well as is done in Bumpy Torus [Catto et al. (1981), Uckan et al. (1981)]. This method has the advantage of preserving axial symmetry of the straight sections. In this method rings of anisotropic electrons are sustained in the transition section by electron cyclotron resonance heating. The electron rings, due to their diamagnetic nature, create magnetic wells and thus provide the required stability. However, the interaction between electron rings and the bulk of plasma causes problems which make it difficult to maintain the rings. Thus a simflar method of stabilization avolding this problem is proposed by Strauss et al. (1985). In this it is proposed to stabilize the torotdally linked mirrors by the ponderomotive force of electromagnetic waves at the lon-cyclotron frequency.

Thus it seems plausible that one may be able to find one of the above mentioned methods or a combination of these to stabilize also the THELMA device. However only a detalled analysis would be able to say anything definitive in this respect. 


\section{Chapter IX}

\section{Summary and Concluston}

Coming to the end, we would like to first summarize the previous chapters and then end the thesis with concluding remarks. We have presented here a scheme for linking straight magnetic mirror sections using simple heltcal links which may be able to preserve the axtal symmetry of the mirrors to a large extent. Axtal symmetry keeps the radial loss small. We have first studied theoretically the magnetic fleld and single particle motion. We expect on the basis of a theoretical analysis that the device would have magnetic fleld lines which form well defined nested magnetic surfaces and that most of the passing particles would remain confined. We also estimate a high rotational transform value for the device, which is not near a low rattonai number multiple of $2 \pi$.

Computationally we studied these aspects using various computer codes. We find that magnetic field lines do form magnetic surfaces and the passing particles are confined within the device to a large extent as expected. We also find that the particles which are trapped in the helical section get lost from the device rapldily. The effect of this on the confinement time as a whole and the density of plasma is given in Appendix-8. The rotational transform is found to be in good agreement wtth the theoretical estimate. We also study the use of noncircular colls in the helical links. It is found that the magnetic surfaces created by noncircular colls have a 0 -shape in the helfcal links, but change to a near ctrcular one in the stratght section. However the 
volume occupled by the outermost magnetic surface decreases. The magnetic well created by the coll system. Which was not optimized in any way, was not sufficient to provide an overall positive average magnetic well. But this can be done by other means as suggested in the prevfous chapter.

We also estimated the maximum $B$ value on the basis of the transverse shift of the plasma column. For a THELMA configuration in which nu magnetic mirrors are present we estimate that a maximum average $B$ value obtainable is $\sim 5.5 \%$, which may mean that with mirrors we would expect $\theta$ values of $15-20 \%$ in the straight section.

We concluded by giving a survey of varlous stability schemes proposed for other linked mirror schemes and show that there is a possibility that one or more of these methods may be sufficlent to provide the stability to THELMA.

Concluding, we would like to say that the possibilty of linking magnetic mirror sections by helical sections is promising. In particular we have shown good confinement of passing particles, which means that helical ilinks do a good job of transporting particles from one straight section to another. We have also shown that moderately high $p$ values can be achleved. There is a definite posstbllity of stabliting the devtce by some means. However, we should try to reduce the loss of trapped particles from the Iinks. Thus at the outset, we need not confine ourselves to only such links. With the level of theoretical understanding we have, we may be able to find linking structures which may have deeper magnetlc wells, reduced charge-neutralization currents and more effictent volume utilization. Such an attempt has been made in the case of the Advanced Stellarator Wendelstein VII-AS [Kissilnger (1981)]. 
which also uses modular colls and has a planar axis with pentagonal shape. We may be able to use modular coils in linking mirrors as well, but they must be optimized very well.

I would personally suggest, a device with simple links having rotational transform (such as helical) and use of the ponderomotive force in the straight section with noncircular or modular colls in the helical section to provide for stablitity. In designing modular colis, attempts should be made to keep the axisymetricity of the straight section as much as possible. 
Appendix A

Mercter Coordinate System

Before we describe Mercter Coordinates we would like to give some commonly used mathematical terms in reference to a 3-dimenstonal curve. For a spatial (3-dimensional) curve, we define (as shown in Fig.

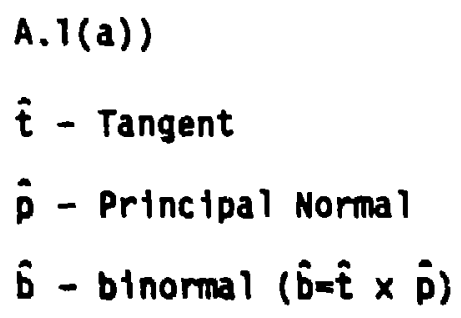

Where $k \equiv$ Curvature

$x \equiv$ Torston

and the dot represents $\frac{\partial}{\partial s}$ (1.e. rate of change along the curve). We define the osculating Plane as the plane containing $\hat{\mathrm{t}}$ and $\dot{\mathrm{p}}$. Then we can physically think the curvature to be the rate at which a curve tends to depart from tts tangent and Iorston to be the rate at which the curve tends to depart from its Osculating plane.

Now we Introduce the Mercter Coordinate system which is a generalization of the cylindrical coordinate system, with an axis that is a spatial (3-dimenstonal) curve. We choose a spatial curve

$$
r=r_{0}(s)
$$

to be the axts of the coordinate system. 


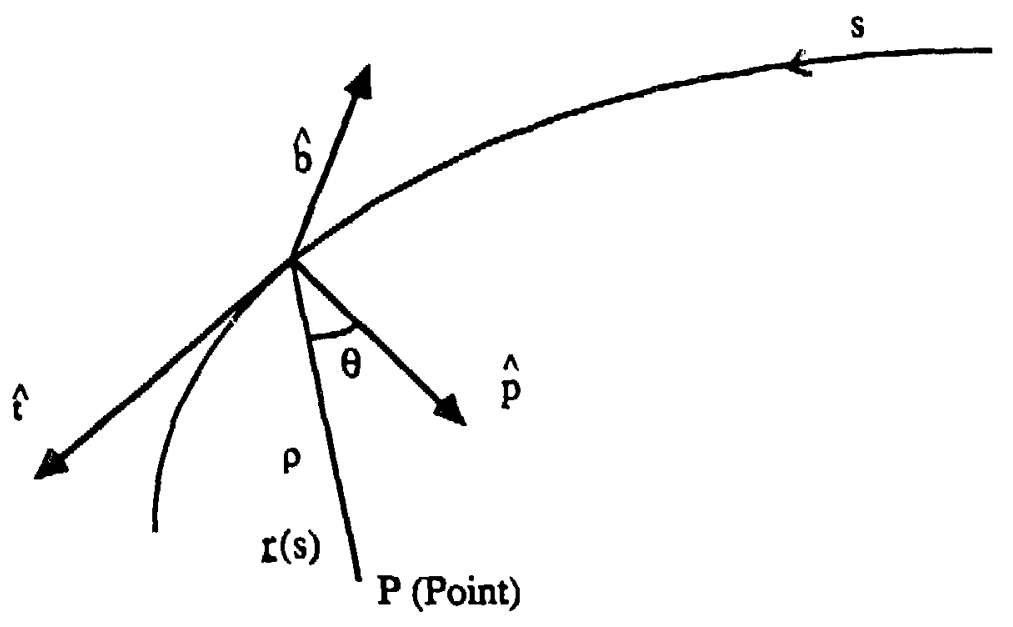

Fig. A.1 (a)

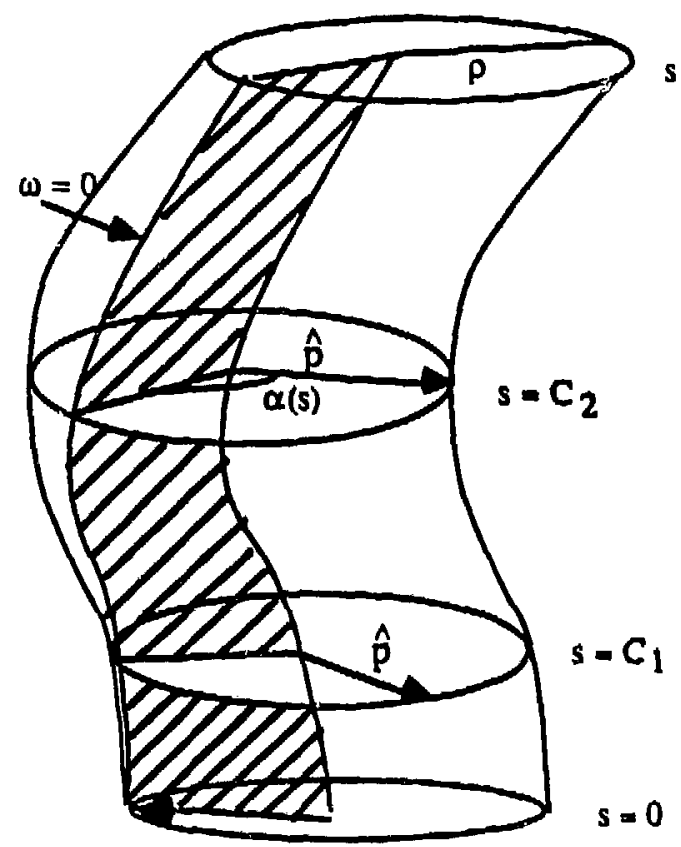

$s=C_{3}$

$\hat{\mathbf{p}}$

Fig. A.1(b)

Fig. A.1(c) 
Let $\rho$ be the distance from the axis; $\theta$ the angle computed from the principal normal, and s the length of arc along the axis computed from some fixed point $s=0$, as shown in Fig. A.1.

In this system the distance increment vector is given as

$$
d r=\hat{e}_{\rho} d \rho+\hat{e}_{\theta \rho}(d \theta+x d s)+\hat{e}_{s}\left(1-k_{\rho} \cos \theta\right) d s
$$

Here $\hat{e}_{\rho}, \hat{e}_{\theta}$ and $\hat{e}_{s}$ denote a set of unit vectors aligned with the three indicated directions. As can be seen the coordinate system in this form is not orthogonal (because of the cross-terms) and thus we define a new angular coordinate $\omega$ as

$$
\begin{aligned}
& \omega=\theta+\alpha(s) \\
& \alpha(s)=\int_{0}^{s} x d s
\end{aligned}
$$

With this definition we have the distance element squared as

$$
d l^{2}=d \rho^{2}+\rho^{2} d \omega^{2}+(1-k \rho \cos \theta)^{2} d s^{2}
$$

For physical quantities we require that they be functions of $\theta$, as $\omega=$ const. plane does not necessarlly close upon ttself after one complete turn around the system, because of rotation of principal normal caused by torsion.

The Laplacian operator in this coordinate system takes the form

$$
\nabla^{2} \phi=\frac{1}{\rho} \frac{\partial}{\partial \rho}\left(h_{s} p \frac{\partial \phi}{\partial \rho}\right)+\frac{1}{\rho^{2}} \cdot \frac{\partial}{\partial \omega}\left(h_{S} \frac{\partial \phi}{\partial \omega}\right)+\frac{\partial}{\partial s}\left(\frac{1}{h_{S}} \cdot \frac{\partial \phi}{\partial s}\right)
$$

Where,

$$
h_{s} \equiv 1-k_{\rho} \cos \theta=1-k(s) \rho \cos [\omega-\alpha(s)]
$$

This coordinate system is very useful for toroidal devices with small curvature. 
Append $1 \mathrm{x}-\mathrm{B}$

Discussion of particles trapred in helical sections

In the simple helical bends investigated here, we find that the particles trapped in the bends seem to get lost fast as shown in Fig. 8.1, where the radial distance of a particle trapped in a heifical section is shown. We feel that this takes place because of the following scenarto. Particles trappped in the helicai bend bourice back and forth in the section. As discussed earlier the particles tend to follow the field lines and the deviation from the fleld lines is due to the curvature and gradient-B drifts. The typical effect of curvature and gradient drift is to move the particles in the direction of the principal normal and this movement does not get affected by the direction of longitudinal motion. Thus a particle feels the drift in the säme direction, regardless of whether it is going back or forth. On the other hand the rotational transform depends upon the direction of transit. Thus a particle going back and forth has the effect of the rotational transform cancelled. Hence the net effect is that trapped particles keap moving outward and then they get ripple trapped and eventualiy get lost. lowever, there are two processes which decrease this loss. One is colltsions, which may detrap the particle and the other is the ambipolar field, which provides sone polotdal drift and reduces the loss to some extent [Hitchon (t) al. (1985)]. Even then most of the particle which get trapped are 10: to the device. However this loss is much lower than the ordinary mirror loss, as the density in the helical section would be considerably lower than the straight sections. The effect of high loss 


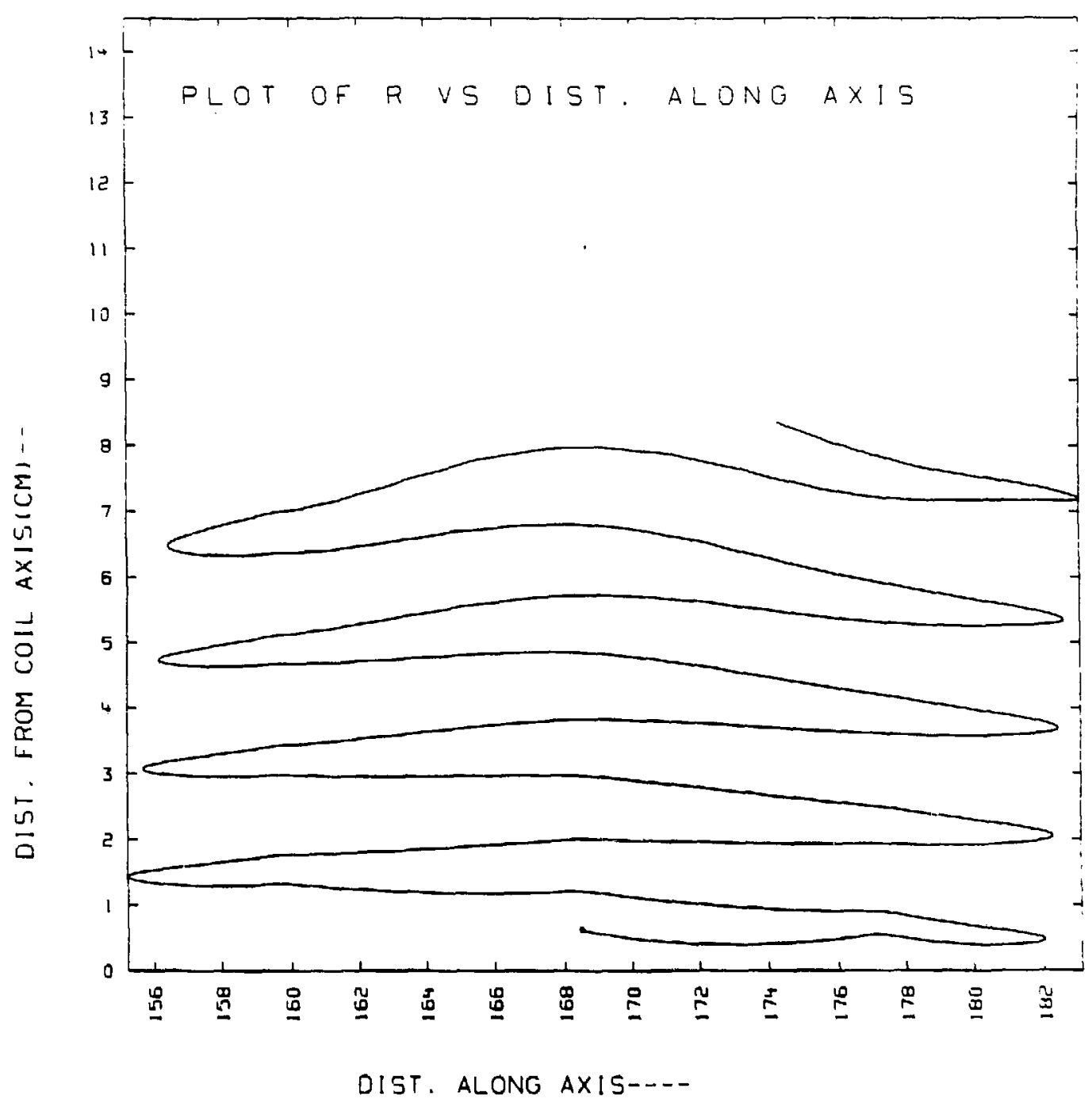

Fig. B. 1 
of particles would always keep the density in the helical section low and thus the trapping rate due to collision would be less also.

A very crude comparison of the ordinary mirror loss and the loss in our saheme can be made as follows. The loss depends on the density of particles in the helical section, the volume of the helical section and the fraction of passing particles subject to loss. We assume that the scattering rate in the long straight mirror sections is fast enough to keep the velocity distrfbution isotroptc, 1.e., to keep the loss-cones fllled. The density $n_{s}$ in these regions is therefore expected to be uniform in space, all the way to the mirror throats. In the helical sections, on the other hand, the densities $n_{h}$ drop to much lower levels because only few trapped particles remain there at any given time, $1 . e$. any mirror ratio $R_{h} \equiv B_{t} / B_{h_{t}}>1$ makes the velocity in the helfcal region iritsotopic, and only the loss-cones are nearly filled. In a first approximation we can estimate that $n_{h}<n_{s} B_{h} / B_{t} \equiv n_{s} / R_{h}$. which is based on the assumption that the passing particles remain on their flux tubes.

If we denote the rate of scattering of a particle near the loss-cone boundary into (or out of) the loss-cone in the straight section by $v_{s}^{l c} ; 1 . e$.

$$
\left.\left.v_{s}^{l c} \equiv n_{s}<0 \quad v\right\rangle_{s}\right] c
$$

Then the rate of trapping of such a particle in the helical sections can be estimated by the following expression:

$$
\begin{aligned}
v_{h}^{t r}=v_{h}^{l c}=n_{h}\langle\sigma v\rangle_{h}^{l c}\left\langle n_{s}\langle\sigma v\rangle_{h}^{l c} B_{h} / B_{t}\right. & \approx n_{s}\left\langle\sigma v v_{s}^{l c} B_{h}^{2} / B_{s} B_{t}\right. \\
& =v_{s}^{l c} B_{h}^{2} / B_{s} R_{h}
\end{aligned}
$$

The latter relations are based on the observation that the distributions 
of particle speeds in the two regions are simflar and that the areas in velocity space of the loss-cone boundaries are inversely proportional to the mirror ratios, so that $\langle\sigma v\rangle_{h}^{l C} / B_{h} \approx\langle 0 v\rangle_{s}^{l c} / B_{s}$. The relation is only approximate because the anisotropy in the helical sections, and particularly the counterstreaming character in velocity space must be expected to introduce an additional deviation.

The total rate of trapping in the helical sections, and hence the upper bound of net particle loss through the mirror throats of the straight sections can now be related directly to the ordinary mirror loss: we define the confinement gain factor

$$
G \equiv \frac{n_{s} V_{s} v_{s}^{l c}}{n_{h} V_{h} V_{h}^{T C}}>R_{h} \frac{L_{s}}{L_{h}} \cdot \frac{B_{h}}{B_{s}} \cdot \frac{v_{s}^{I c}}{v_{h}^{T c}} \approx R_{h}^{2} L_{s} / L_{h}
$$

where the magnetic flux in the straight and helical sections is assumed equal and related to the respective volumes $V_{s}$ and $V_{h}$, and lengths $L_{s}$ and $L_{h}$ by the equality $B_{s} V_{s} / L_{s}=B_{h} V_{h} / L_{h}$, and relation (2) is used to eliminate the scattering rates. We see that with $R_{h}=2$ and $L_{s}>5 L_{h}$ a sizable gain in confinement time is expected.

Here, we also show an approach to finding the effect of how the loss of particles from the hellcal bends affects the density in various sections. However some parameters here, and in particular the various rates, have been left unknown, which means that they must be determined by other means. A proper calculation in this direction would use the Fokker-planck equation. We first define the notations as follows:

$$
\begin{aligned}
& p \rightarrow \text { Passing particle, } \\
& t \rightarrow \text { Trapped particle, }
\end{aligned}
$$


c $\rightarrow$ Curved section,

$s \rightarrow$ Straight section,

$k_{a b}^{c} \rightarrow$ Reaction rate constant between ' $a$ ' and ' $b$ ' leading to creation of type ' $c$ ' particle.

$S \rightarrow$ Source rate,

$R_{t}^{l} \rightarrow$ Loss rate of trapped particle,

$v \rightarrow$ valume.

Then we make the following assumptions:

(1) Assume that the electron distribution is Maxwellian.

(2) Assume that the ion distribution is Maxwellian in the straight section, then passing particles as fraction of total population in the straight section as function of mirror ratio $M$, is given as

$$
f(H)=1-r\left(1-1 / M^{2}\right)
$$

(3) Assume there is no loss from the stralght section. All the loss that takes place is through the curved section. which may be due to toroidal effect or ripple effect etc.

Under these conditions the rate of change of densities in the steady state in the straight section and curved section, gives us the following two equations:

$S-n_{p}^{2} v_{c} k_{p p}^{t}+n_{p} n_{t} v_{c}\left(k_{p t}^{p}-k_{p t}^{t}\right)+n_{t}^{2} v_{c} k_{t t}^{p}=0$

$n_{p}^{2} v_{c} k_{p p}^{t}-n_{p} n_{t} v_{c}\left(k_{p t}^{p}-k_{p t}^{t}\right)-n_{t}^{2} v_{c} k_{t t}^{p}-R_{t}^{2} v_{c} n_{t}=0$;

Solving these two we get the number density of particles trapped in the curved section as, 
$n_{t}=\frac{S}{R_{t}^{2} V_{c}}$

and we get a quadratic equation for $n_{p}$ as,

$n_{p}^{2} v_{c} k_{p p}^{t}-\frac{n_{p} S\left(k_{p t}^{p}-k_{p t}^{t}\right)}{R_{t}^{l}}-S\left[1+\frac{S k_{t t}^{p}}{\left(R_{t}^{2}\right)^{2} v_{c}}\right]=0$

Thts quadratic equation can always be solved for $n_{p}$, but if we make the assumption that (which is simply an assumption of symmetry)

$$
k_{p t}^{p}=k_{p t}^{t}
$$

Then we get,

$n_{p}^{2}=\frac{1}{v_{c} k_{p p}^{t}}\left\{S\left[1+\frac{\left.\left.s k_{t t}^{p}\right]\right\}}{\left(R_{t}^{l}\right)^{2} v_{c}}\right.\right.$

and when

$R_{t}^{2} \rightarrow \infty, n_{p}^{2}=\frac{s}{y_{c} k_{p D}^{t}}$

In other words, we may put the result as follows:

(a) for $\frac{S k_{t t}^{D}}{\left(R_{t}^{2}\right)^{2} V_{c}} \ll 1, \quad n_{D}^{2}=\frac{S}{V_{c} k_{P D}^{t}}$ (does not depend on $R_{t}^{2}$ )

(b) for $\frac{S k_{t t}^{p}}{\left(R_{t}^{2}\right)^{2} v_{c}} \gg 1, \quad n_{p}^{2}=\frac{S^{2} k_{t t}^{p}}{\left(R_{t}^{2}\right)^{2} v_{c}^{2 k} k_{p p}^{t}}$ (depends strongly on $R_{t}^{2}$ )

This result can thus be interpreted to mean the following. In the case when the loss rate from the helfcal bends is large, the situation does not change much by reducing the loss rate by a small amount. This is only effective when we reduce it so much that condition (b) is 
satisfied. However, if the condition (b) is already satisfied, then any smal1 reduction in loss rate improves the density very we 11 . 
Appendix C

\section{Generation of coils}

For the generation of the coll to create a trianlgular helically linked system we used a computer program. As described earlier we use a generating cylinder to create the helical links. The straight segments, when projected onto the plane created by their midpoints, form segments of an equilateral triangle (See Fig. 3.1). The midpoints of the helical links also lie in this plane. Once we specify the radius of the generating cylinder and the placement of the generating cylinder with respect to the equilateral trlangle, we have spectfled all the parameters required for generating the geometrical axis of the device. These parameters in turn determine the curvature and the torsion of the helical links. The actual colls which may be circular or noncircular may be then placed along the axis as desired.

We show some geometric calculation here to establish the relationship between varfous parameters of interest. In Fig. C.I we show some parameters needed to specify a helix. Let the length of the side of the equilateral trlangle IJK be $p($ see Fig. C.2 and C.3). In Fig. C.2 we show the plan view of the equilateral triangle from above. In Fig. C.3 the view is along the axts of the generating cylinder. Then we have

$$
\begin{aligned}
& I J=p \text { (Length of side) } \\
& L J=\frac{\sqrt{ } D}{2} \text { (Length of perpendicular bisector) } \\
& C M=\frac{p}{2 \sqrt{3}} \text { (Length from center to middle of the side) } \\
& C O^{\prime}=C M \operatorname{Cos}\left(\frac{\pi}{3}\right)=\frac{C M}{2}=\frac{D}{4 \sqrt{3}} \\
& C J=\frac{P}{\sqrt{3}} \\
& O O^{\prime \prime}=R_{m} \cos \theta \text {, where } \theta \text { is the angle between } R_{m} \text { and } 00^{\prime \prime} .
\end{aligned}
$$



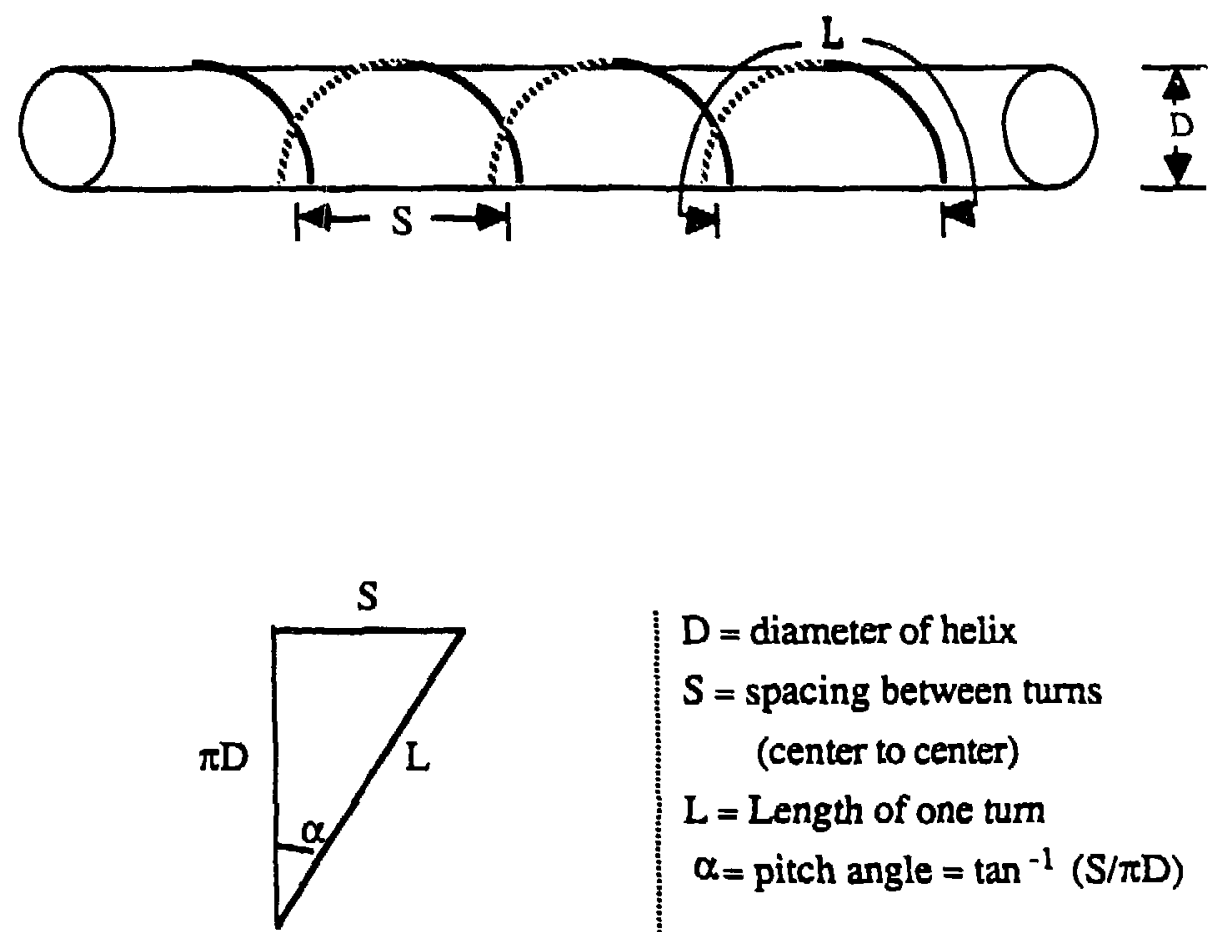

$\mathrm{D}=$ diameter of helix

$S=$ spacing between turns (center to center)

$\mathrm{L}=$ Length of one tum

$\alpha=$ pitch angle $=\tan ^{-1}(\mathrm{~S} / \pi \mathrm{D})$

XBL $8610-3720$

Fig. C.1: Parameters associated with helix 


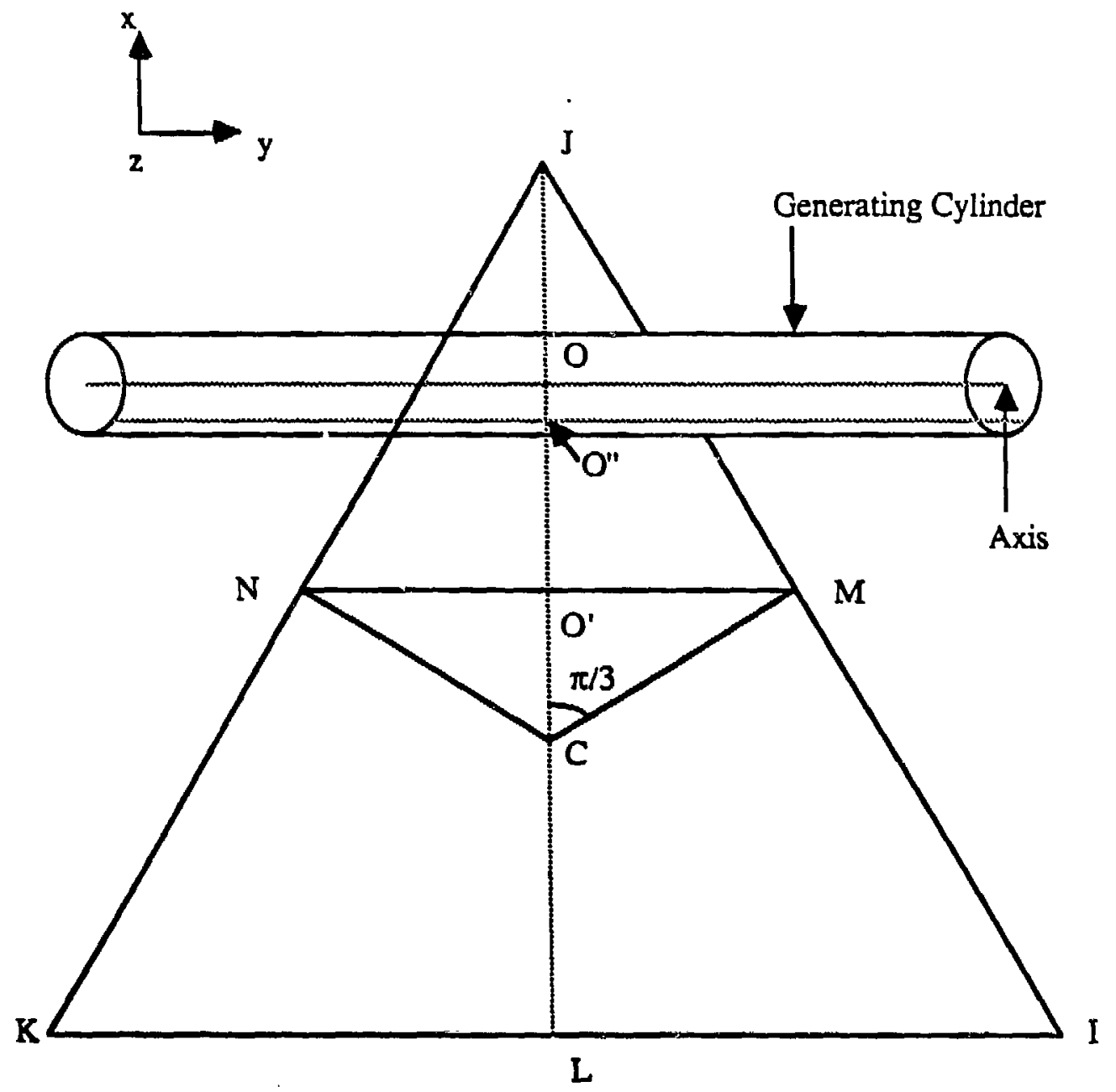

XBL 8610-3721

Eig. C.2 


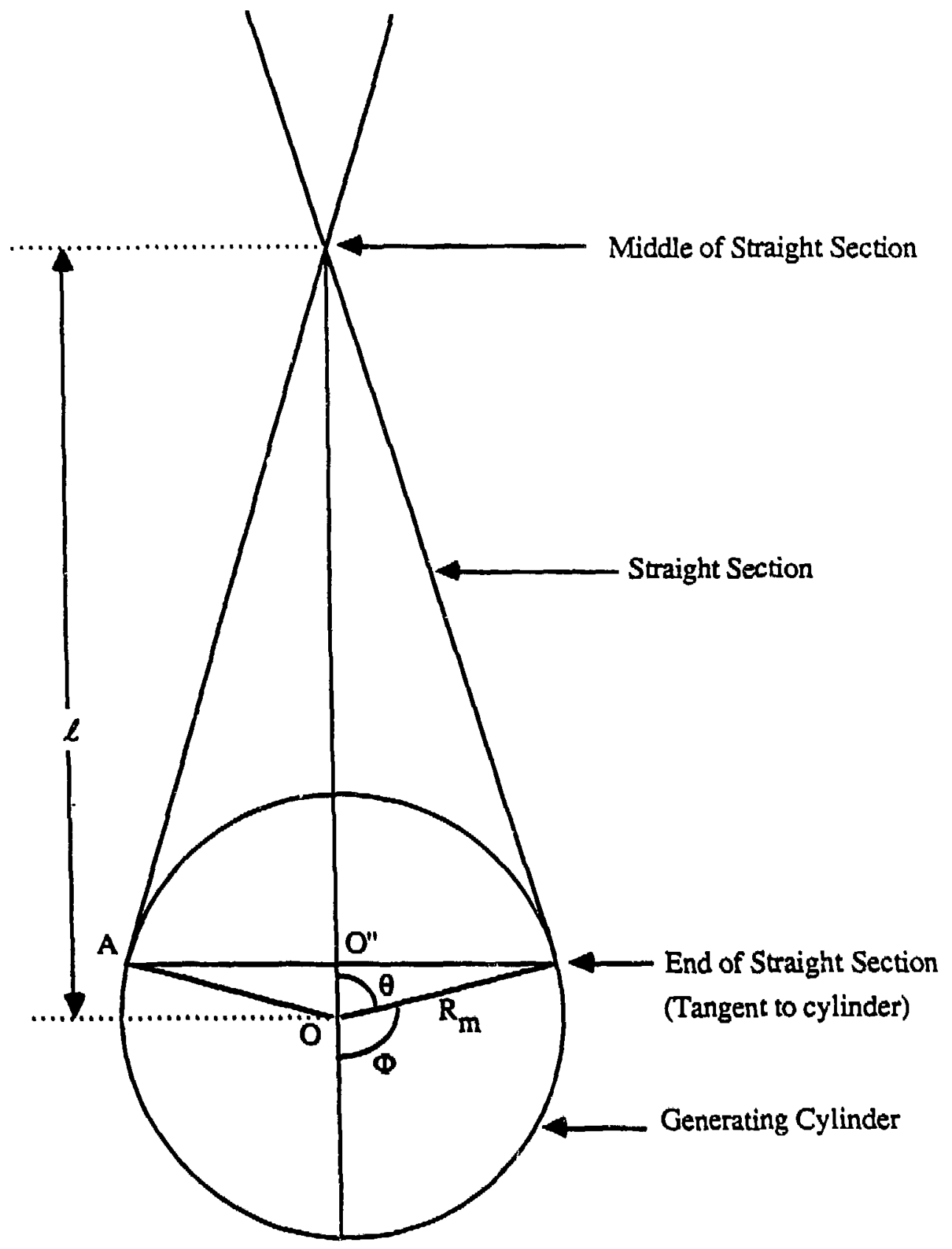

XBL $8610-3722$

Eig. C. 3 


$$
\begin{gathered}
0^{\prime \prime} 0 J=C J-C^{\prime \prime}=C J-C O^{\prime}-0^{\prime} 0+00^{\prime \prime} \\
=\frac{p}{\sqrt{3}}-\frac{p}{4 \sqrt{3}}-2+R_{m} \cos \theta
\end{gathered}
$$

Then,

Since $\tan \frac{e}{6}=\frac{A Q^{\prime \prime}}{0^{\prime \prime J}}$, we get

$\left.A O^{n}=0^{n}\right] \tan \frac{e}{6}=\left[\frac{\mathrm{D} V}{4}-2+2 \cos ^{2} \theta\right] \tan \frac{e}{6}$

$$
=\left[\frac{\mathrm{DV} 3}{4}-2 \sin ^{2} \theta\right] \tan \frac{e}{6}
$$

$\tan ^{-1}\left[\Phi \cdot R_{m} / A O^{\prime \prime}\right]=$ a (by definition, pitch angle of the helix,

$$
\text { see Fig. (.1); }
$$

In our case, $a=\frac{e}{3}$; so that

$\tan a=\tan \frac{e}{3}=\Phi \cdot R_{m} / A O^{\circ}$

From this we get,

$$
\left[\frac{\mathrm{p} \sqrt{3}}{4}-2 \sin ^{2} \theta\right] \tan \frac{e}{6}=R_{m}(r-\theta) / \tan \frac{e}{3}
$$

Thus we obtain a relation linking the length of the side of the equilateral triangle (p), the displacement of the generating cylinder from the line meeting the mid-points of the triangle (l) and the radius of generating cylinder $\left(R_{m}\right)$ as follows [Note that $\theta$ is defined as $\left.\cos ^{-1}\left(R_{m} / 2\right)\right]$ :

$$
\left[\frac{\mathrm{P} v 3}{4}-2 \sin ^{2} \theta\right]=R_{m}(\pi-\theta)
$$

This also tells us that we have only two independent parameters which in turn determine the torsion and the curvature of the linking. Thus to determine the complete coll configuration we need to specify the radius, width and thickness of the coll, the current in the colls and the radial shift of the helix (in case a shifted THELMA configuration is required). We also need to sperify the number of colls in the helical and straight sections. Once these are specified the program calculates the position of various coils along the straight and the helical sections. It also 
calculates the Euler Angles to specify the orientations of the coil. The plane of the cotl is assumed to be perpendicular to the axis. The symmetry of the device is used to construct the various sections after $\frac{1}{6}$ of the colls have been calculated. The program has options to have output files created in formats compatible with TIBRO-X [Foote (1976)] and HELIAC [Ehrhardt (1985)] computer programs.

The program also has the option to specify noncircular colls, having the same position and orientation as the circular coils. The parameters needed to specify the nonctrcular colls are describad as follows.

Specification of the noncircular coil is done using Monticello [Monticello et al. (1984)] bean parameters, which describe typically a coll of bean shape. However by proper use of parameters many other shapes can also be obtained. In terms of cylindrical coordinate $\theta$, the $x$ and the $y$ coordinates of the coil are given as-

$$
\begin{aligned}
& x=r \cos \sigma+x^{\prime} \\
& y=r \sin \sigma
\end{aligned}
$$

where,

$$
\begin{aligned}
& \sigma=B \sin (\pi-\theta) \\
& r=a[1-b \cos (\pi-\theta)]
\end{aligned}
$$

Here $a, b, B$ and $x^{\prime}$ are the parameters which determine the shape of the coil. We assume that the noncircular colls have the same area as the circular colls and also that the noncircular colls are centered at the center of the circular colls. The parameters $x^{\prime}$ and a are determined from these two assumptions, so we need to give only two parameters $B$ and $b$ to specify the shape of the colls. We also need to specify the orientation of the coils local coordinate system, which we do through another angle 
parameter. We show in Fig. C.4(a) - (f), the parameters we used to chang = the shape of the colls from bean shape to circular gradually. Also shown are corresponding theoretical shapes, the approximation used for numerical calculations and the circular coil as reference. The theoretical shape is shown by the solid line and the approximation used by the dashed line. The angle of the minor axis of the coll with the principal normal of the geometric axis was kept fixed. 


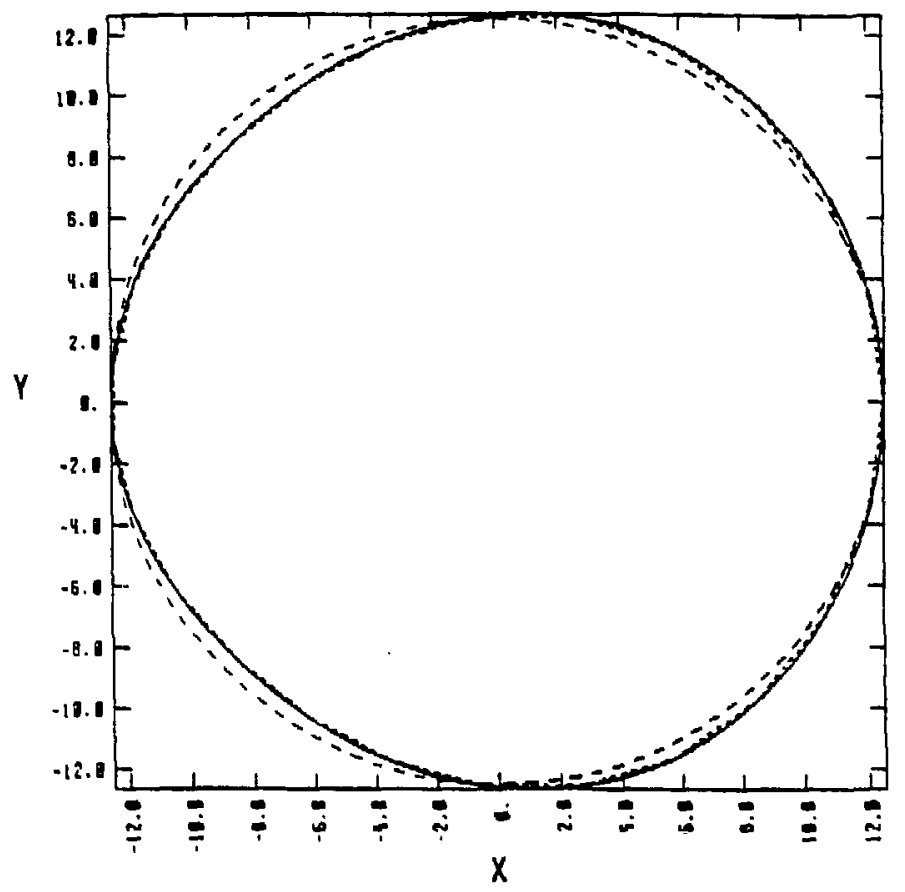

Fig: $C .4(a): b=0.2, B=0.2$

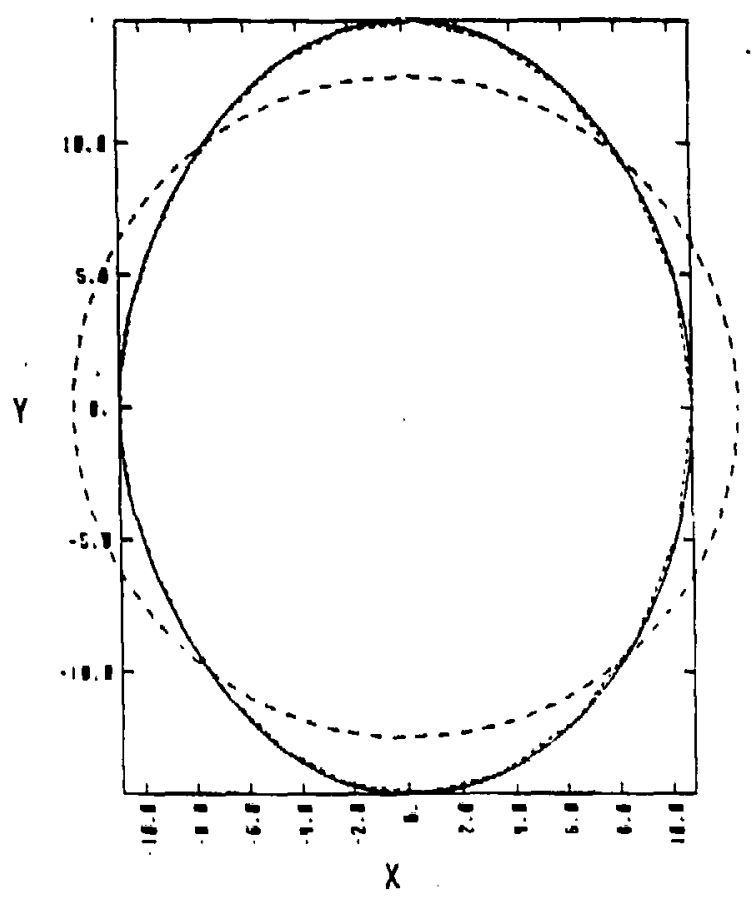

Fig. $C .4(b): b=0.3, B=0.4$ 


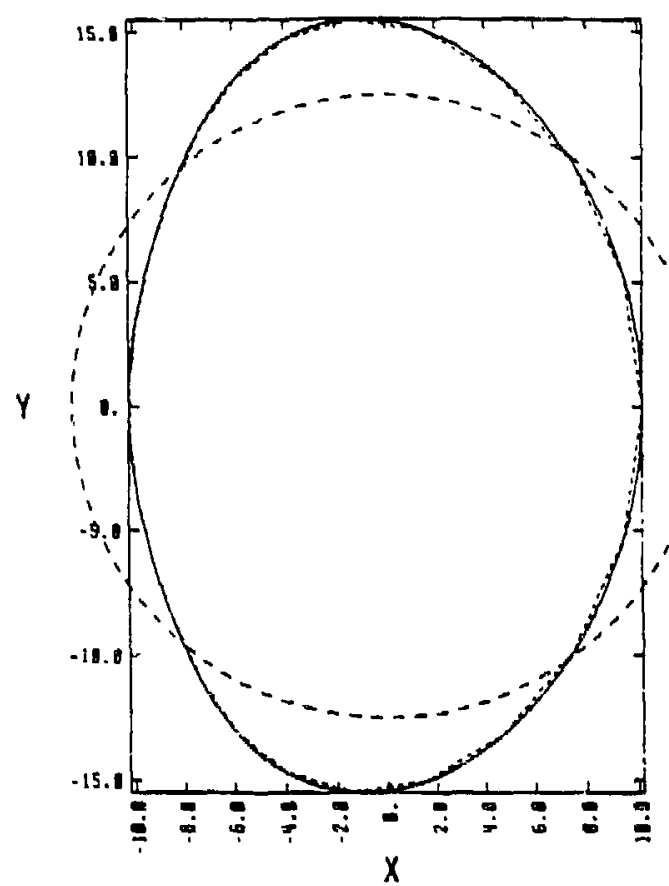

Fig. $C .4(c): b=0.4,8=0.6$

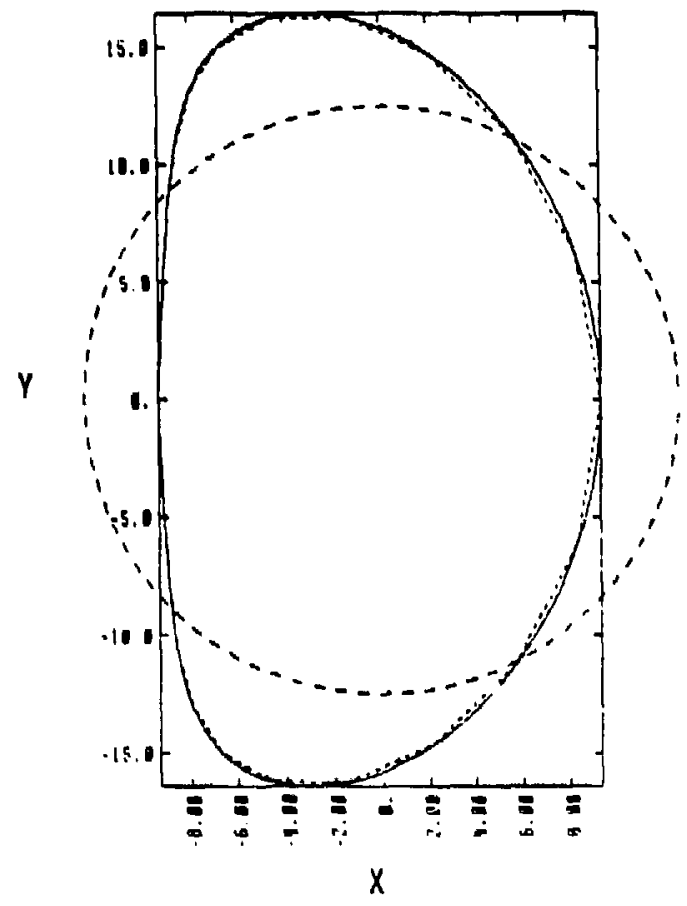

Fig. $C .4(d): b=0.5, B=0.9$ 


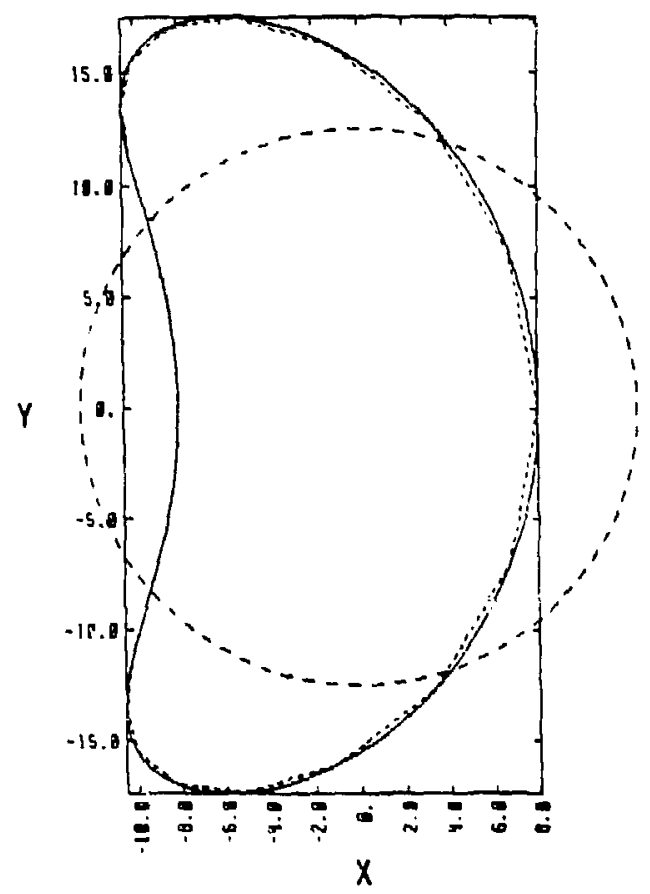

Fig. $C .4(e): b=0.5, B=1.2$

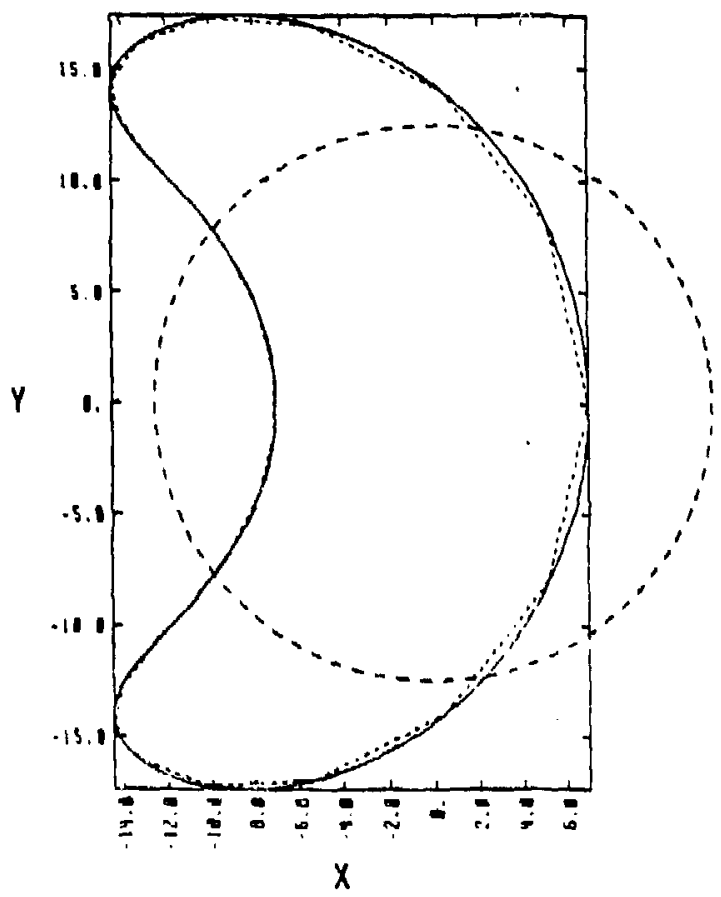

Fig. $C .4(f): b=0.5, B=1.6$ 


\section{References}

Abt, N. E. and Kunkel, W. B.; Bul1. Am. Phys. Soc. 28, 1033(1983).

Arsenin. V. V. , V. M. Glagolev, B. B. Kadomtsev, et al., in Plasma Phys. and Contr. Fus. Res., (Proc. 9th Int. Conf. Baltimore, 1982), Vol. III. IAEA, Vienna, 159 (1983).

Bishop, A. S. Project Sherwood. Addison-Wesley Publishing Company, 1958. Catto, P. J. , et al., in Plasma Phys. and Contr. Nuc. Fus. Res. (Proc. 8th Int. Conf. Brussels, 1980) Vo1. 1, IAEA, Vienna, 821 (1981).

Cordey, J. G. and C. J. H. Watson, Fusion Reactor Design Problems (Proc. IAEA Workshop, 1974), Nucl. Fustion Spec. Supp. (1974) 199.

Cordey, J. G. and C. J. H. Watson, Plasma Physics and Controlled Fuston

Research (Proc. 5th Int. Conf. Tokyo, 1974) 2, IAEA, Vtenna (1975) 643.

Dawson, J. H. . H. P. Furth and F. H. Tenney; Stellarator-Mirror Machine Target Reactor, MATT-841, Princeton (May 1971).

E.hrhardt, A., Princeton Plasma Physics Laboratory (Private Communication). (1985).

Foote, J., "TIBRO-X: a code to compute trajectorles of charged particles In magnetic fields", UCRL-52189 (1976).

Glagolev, V. M. et. al., in 10th European Conference on Controlled

Fustion and Plasma Physics. European Physical Society.(1981) $\varepsilon-8,389$.

Glagolev, V. H., A. V. Dobryakov, S. L. Lazarev and B. A. Trubnikov in Sov. J. Plasma Phys. 10 (1984) 415.

Glagolev, V. M. B. A. Trubnikov and Yu. N. Churin, Nuclear Fusion 25 (1985) 891. 
Grad, H. , Phys. Fluids 10, 137 (1967).

Guest, G. E. W. H. Farr, and R. A. Dory, Phys. Fluids 13, 3038 (1970).

Ha11, L. S. and B. McNamara; Bull. Am. Phys. Soc. II, 19 (1974) 872 .

Hastie, R. J. and C. J. H. Watson, Nuclear Fuston 17, 1153 (1977).

Hitchon, W. N. G., et al., J. Plasma Physics, 34, part 2,327 (1985).

Jukes, J. D. Plasma Physics $\underline{6}, 84$ (1964).

Karney, C. F.,"COIL3: A set of magnetlc field and vector potential calculation for magnetic fleld elements", NMFECC(1985).

Killeen, J. H. P. Furth and M. M. Rosenbluth; Bull. Am. Phys. Soc. Il (1966) 549.

Kissilnger, J. F. Rau and H. Wobig, in Proc. of the 12th Symp. on Fusion technology (SOFT) Julich, 1051 (1981).

Kunke1, W. B., Bull. Am. Phys. Soc. 28, 1033(1982)

Leontovich, M. A., (Ed.), in Reviews of Plasma Physics, Vol. 5. Consultants Bureau, New York (1966).

Logan, B. G., et al., "Mtrror Advanced Reactor Study," UCRL-53333 (1983). Makurin, S. V. and A. B. Mikhatlovski1, Sov. J. Plasma Phys. 10, 171 (1984).

Mikhallovski1, A. B. Preprint IAE-3120/6, I. V. Kurachatov Institute of Atomic Energy, Moscow, 1983.

Montice 110, D. A., Dewar, R. L., Furth, H. P., Retman, A., Phys. Flutds 27. (1984) 1248 .

Ohasa, K. and K. Ikuta; Nuc lear Fusion 17, 473 (1977).

Palumbo, D.. II Nuovo Cimento, 53B, 507 (1968)

Shafranov, V. D. . Nucl. Fusion 17, 253 (1968).

Shafranov, V. D. Phys. Fluids $\underline{26}$ (2), 357 (1983). 
Solovev, L. S. and V. D. Shafranov in Reviews of Plasma Phystcs (M. A. Leontovich, Ed.). Consultants Bureau, New York, Vol. 5 (1967) 3. Spitzer, L. Jr., Phys. Fluids 1, 253 (1958).

Strauss, H. R. . L. Friedland and M. Kishinevsky, Phys. Fluids $\underline{58(12),}$ 3594 (1985).

Teller, E. ,(Ed.), Fuston, Vol. I Part A, Academtc Press, p. 2, 1981. Trubnikov, B. A. and V. M. Glagolov in Fiz. Plazmy 10 (1984) 288.

Trubnikov, B. A. et al. in Fiz. Piazmy il (1985) 155.

Uckan, N. A. et a 1.. in Plasma Phys. and Contr. Nuc. Fus. Res. (Proc. Bth Int. Conf. Brusseis, 1980) Vo1. 1. IAEA, Vienna, 831 (1981).

Volkov, T. F. A. V. Dcoryakov and A. B. Trubntkov, Nuclear Fuston 25 891 (1985).

Whiteman, K. J. et al. in Plasma Phys, and Contr. Nuc. Fus. Res. (Proc. 2nd Int. Conf. Culham, 1965), Vol. I, IAEA, Vienna (1966) 145. Yoshikawa, M., Nuclear Fusion, 25, 205(1985). 
This report was done with support from the Department of Enercy. Any conclusions or opinions expressed in this report represent solely those of the author(s) and aod nocesurily those of The Rezente of the Univeraity of Califorain, the Lawrence Berteley Lboratory or the Depurtment of Eneray.

Reference to a company or product anme does not imply approval of recommendation of the product by the Univeraity of Califomin or the U.S. Department of Energy to the exclusion of others that my be suilable. 